\title{
Estabilidade de ondas viajantes para a equação de Schrödinger de tipo cúbico com dois pontos simétricos de interação
}

Luis Andrés Rosso Cerón

\author{
TESE APRESENTADA \\ $\mathrm{AO}$ \\ Instituto DE MATEMÁtica E EstatísticA \\ DA \\ Universidade DE SÃo PaUlo \\ PARA \\ OBTENÇÃ DO TÍTULO \\ DE \\ Doutor EM CiÊnCIAS
}

\author{
Programa: Matemática \\ Orientador: Prof. Dr. Jaime Angulo Pava
}

Durante o desenvolvimento deste trabalho o autor recebeu auxílio financeiro da Fundação de Amparo à Pesquisa do Estado de São Paulo, processo FAPESP no. 2010/52665-8 


\section{Estabilidade de ondas viajantes para a equação de Schrödinger de tipo cúbico com dois pontos simétricos de interação}

Esta versão trata-se da tese original do aluno Luis Andrés Rosso Cerón.

Comissão Julgadora:

- Prof. Dr. Jaime Angulo Pava - IME-USP

- Prof. [Orlando Lopes - IME-USP]

- Prof. [Felipe Linares - IMPA]

- Prof. [Fabio Natali - UEM]

- Prof. [Ademir Pastor - IMECC-UNICAMP] 


\section{Resumo}

Rosso Cerón, L. A. Estabilidade de ondas viajantes para a equação de Schrödinger de tipo cúbico com dois pontos simétricos de interação. 2015. $80 \mathrm{f}$. Tese (Doutorado) - Instituto de Matemática e Estatística, Universidade de São Paulo, São Paulo, 2015.

Este trabalho consiste, fundamentalmente, em estabelecer de forma analítica a existência e estabilidade orbital de soluções standing-wave de tipo "peakon", para a seguinte equação de Schrödinger com dois pontos de interação, determinados por duas deltas de Dirac centradas nos pontos $x= \pm c(\mathrm{NLS}-\delta)$,

$$
i \partial_{t} u(x, t)+\partial_{x}^{2} u(x, t)+Z\left[\delta_{c}(x)+\delta_{-c}(x)\right] u(x, t)=-|u(x, t)|^{2} u(x, t),
$$

onde $u: \mathbb{R} \times \mathbb{R} \longrightarrow \mathbb{C}, Z \in \mathbb{R}$ e $\delta_{c}$ é a distribuição delta de Dirac agindo em $x=c>0$, a saber, para $\psi \in H^{1}(\mathbb{R}),\left\langle\delta_{c}, \psi\right\rangle=\psi(c)$. Para as soluções standing waves (ondas estacionárias) associadas à equação (1), i.e., $u(x, t)=e^{i \omega t} \Phi_{\omega}(x)$, mostramos que é possível determinar $\mathrm{o}$ perfil $\Phi_{\omega}(x)$ da seguinte maneira: entre os pontos $c$ e $-c$ o perfil $\Phi_{\omega}$ admite, pelos menos, duas funções suaves e positivas dadas pelas funções elípticas de Jacobi conhecidas como dnoidal e cnoidal. Já para $c<|x|$, o perfil $\Phi_{\omega}$ coincide com uma determinada translação do soliton-perfil "secante hiperbólica" (é bem conhecido na literatura que o perfil secante hiperbólica está associado à equação (1), no caso em que $Z=0$ ).

De fato, mostramos que para o caso $Z>0$ é possível ajustar, entre os pontos de interação $c$ e $-c$, um perfil periódico de tipo dnoidal; e para o caso $Z<0$ mostramos como é construído entre os pontos de interação um perfil de tipo cnoidal. Uma questão crucial que surge no problema da existência de um perfil conveniente é aquela relacionada com a localização do ponto de interação $c>0$. A maneira como respondimos a esta questão foi, de fato, determinante para a obtenção do nosso resultado de estabilidade/instabilidade. Isto se deve a que permitiu o uso de técnicas conhecidas na literatura no desenvolvimento do trabalho. En concreto, a escolha da localização do ponto de interação $c$, faz com que a segunda derivada do perfil $\Phi_{\omega}$, seja contínua neste ponto.

Baseados em argumentos da teoria de Floquet, teoria de representação de formas bilineares, teoria de extensão de operadores simétricos e a teoria de perturbação analítica para operadores lineares, bem como nos resultados desenvolvidos por Weinstein e Grillakis\&Shatah\&Strauss, mostramos resultados sobre a estabilidade/instabilidade orbital dessas ondas. Mais precisamente, mostramos que aquelas com um perfil dnoidal são instáveis e aquelas um perfil cnoidal são estáveis. Além disto, estudamos o problema de Cauchy para (1) no espaço de energia $H^{1}(\mathbb{R})$. Para tanto, usaremos informações do espectro do operador com interações pontuais

$$
-\Delta_{ \pm c, Z}=-\frac{d^{2}}{d x^{2}}-Z\left[\delta_{c}+\delta_{-c}\right]
$$

o qual representa formalmente uma das famílias de extensões auto-adjuntas do operador 
simétrico

$$
\left\{\begin{array}{l}
-\Delta=-\frac{d^{2}}{d x^{2}} \\
D(-\Delta)=\left\{f \in H^{1}(\mathbb{R}) \cap H^{2}(\mathbb{R}-\{ \pm c\}): f( \pm c)=0\right\} .
\end{array}\right.
$$

Palavras-chave: Equação de Schrödinger não-linear, potencial delta de Dirac, funções elípticas de Jacobi, perturbação analitica, teoria de Floquet, estabilidade orbital. 


\section{Abstract}

Rosso Cerón, L. A. Stability of travelling waves for the Schrödinger's equation of cubic type with double symmetric delta-interactions wells . 2015. $80 \mathrm{f}$. Tese (Doutorado) - Instituto de Matemática e Estatística, Universidade de São Paulo, São Paulo, 2015.

This work consists mainly in establishing an analytical way the existence and orbital stability for the standing-wave solutions of "peakon"type of the following Schrödinger equation with two points of interaction, determined by two Dirac's delta centered at the points $x= \pm c$ $(\mathrm{NLS}-\delta)$,

$$
i \partial_{t} u(x, t)+\partial_{x}^{2} u(x, t)+Z\left[\delta_{c}+\delta_{-c}\right] u(x, t)=-|u(x, t)|^{2} u(x, t),
$$

where $u: \mathbb{R} \times \mathbb{R} \longrightarrow \mathbb{C}, Z \in \mathbb{R}$ and $\delta_{c}$ is the Dirac's delta distribution in $x=c>0$, namely, for $\psi \in H^{1}(\mathbb{R}),\left\langle\delta_{c}, \psi\right\rangle=\psi(c)$. For the standing-wave solutions associated to equation (2), i.e., $u(x, t)=e^{i \omega t} \Phi_{\omega}(x)$, we show that is possible to determine the profile $\Phi_{\omega}(x)$ as follows: between the points $c$ and $-c$, the profile $\Phi_{\omega}$ admits at least two smooth positive functions given by the Jacobi elliptic functions of dnoidal and cnoidal type. For $c<|x|$, the profile $\Phi_{\omega}$ coincides with an specific shift of the soliton-profile hiperbolic secant profile (it is well-known in the literature that the hiperbolic secant profile is associated to the equation (2) for the case $Z=0$ ).

Indeed, we show for the case $Z>0$ that it is possible to determine a periodic dnoidal profile between the points $c$ and $-c$. On the other hand, for the case $Z<0$ we establish a periodic cnoidal profile between the points $c$ and $-c$. A crucial question arises in the problem of the existence of a suitable profile is the one related to the location of the interaction point $c>0$. This question was crucial to the achievement of our stability/instability result. In fact, the choice of location of the interaction point $c$ implies that the second derivative of the porfile $\Phi_{\omega}$ is continuous at $c$.

The stability/instability theory of these specific profiles are based on the analityc perturbation theory and the framework developed by Weinstein and Grillakis\&Shatah\&Strauss. More precisely, we show that those ones with a dnoidal profile are unstable and those ones with a cnoidal profile are stable. In addition, we study the Cauchy problem in the energy space $H^{1}(\mathbb{R})$ for equation (2). For this purpose, it is necessary to study the spectrum of the operator

$$
-\Delta_{ \pm c, Z}=-\frac{d^{2}}{d x^{2}}-Z\left[\delta_{c}+\delta_{-c}\right]
$$

This operator can be understood as the family of self-adjoint extension of the symmetric operator

$$
\left\{\begin{array}{l}
-\Delta=-\frac{d^{2}}{d x^{2}} \\
D(-\Delta)=\left\{f \in H^{1}(\mathbb{R}) \cap H^{2}(\mathbb{R}-\{ \pm c\}): f( \pm c)=0\right\} .
\end{array}\right.
$$

Keywords: Non-linear Schrödinger equations, orbital stability, Dirac's delta potential, Jacobian elliptic functions, analytic perturbation, teoria de Floquet, orbital stability. 


\section{Conteúdo}

Lista de Símbolos $\quad$ vii

Introdução $\quad$ ix

1 Preliminares $\quad 1$

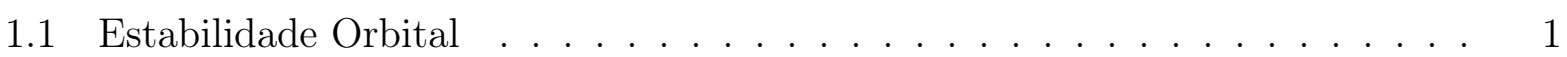

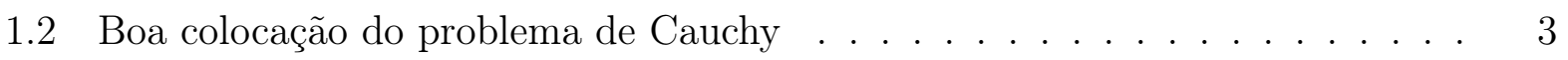

1.3 Interações pontuais . . . . . . . . . . . . . . . . . . . . 4

1.4 Funções elípticas de Jacobi . . . . . . . . . . . . . . . . . . . . . . 6

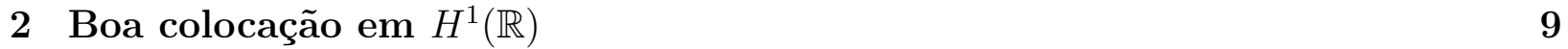

2.1 O operador $H_{Z} \ldots \ldots \ldots \ldots \ldots \ldots$

2.2 Teoria de boa colocação global . . . . . . . . . . . . . . . . . . 11

3 Existência de soluções de tipo peak-standing waves $\quad 13$

3.1 Existência de um perfil periódico entre os pontos de interação . . . . . . . . 14

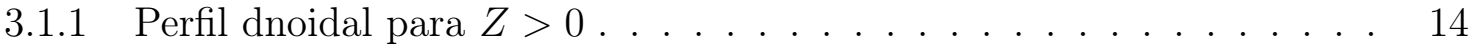

3.1.2 Perfil cnoidal para $Z<0 \ldots \ldots \ldots \ldots$. . . . . . . . . . . . . . . .

4 Teoria de Estabilidade $\quad 35$

4.1 Análise espectral dos operadores $\mathcal{L}_{1, Z, c}$ e $\mathcal{L}_{2, Z, c} \ldots \ldots \ldots \ldots$

4.2 Índice de Morse para $\mathcal{L}_{1, Z, c} \ldots \ldots \ldots \ldots \ldots$. . . . . . . . . . . . . . . . . . . . . . . . . . . . . . .

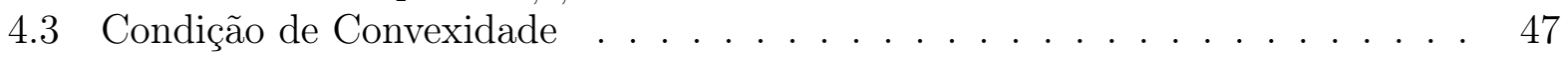

4.4 Prova do Teorema $0.2 \ldots \ldots \ldots \ldots$. . . . . . . . . . . . . . . . 50

$\begin{array}{lll}5 & \text { Estudos futuros } & 53\end{array}$

6 Apêndice $\quad 55$ 


\section{Lista de Símbolos}

- Denotaremos por $\mathbb{N}, \mathbb{R}$ e $\mathbb{C}$ o conjunto dos números naturais, reais e complexos, respectivamente. Pelo símbolo $\mathbb{R}^{+}$denotaremos ao conjunto dos números reais positivos, i.e., $\mathbb{R}^{+}=(0, \infty)$.

- Para $f, g \in L^{2}(\mathbb{R}),\langle f, g\rangle_{2}=\int_{\mathbb{R}} f(x) \overline{g(x)} d x$ denota o produto interno usual no espaço $L^{2}(\mathbb{R})$.

- Para $f \in L^{2}(\mathbb{R}),\|f\|_{2}=\sqrt{\langle f, f\rangle_{2}}$ denota a norma em $L^{2}(\mathbb{R})$.

- $H^{m}(\mathbb{R})=\left\{\psi \in S^{*}(\mathbb{R}):\left(1+\xi^{2}\right)^{\frac{m}{2}} \widehat{\psi}(\xi) \in L^{2}(\mathbb{R})\right\}$, onde $m=1$ ou 2 , e $\widehat{\psi}$ é a transformada de Fourier da distribuição $\psi$. $S^{*}(\mathbb{R})$ é o espaço das distribuições temperadas em $\mathbb{R}$. Este conjunto coincide com o dual topológico do espaço de Schwartz,

$$
S(\mathbb{R})=\left\{f \in C^{\infty}(\mathbb{R}): \sup _{x \in \mathbb{R}}\left|x^{m} f^{(n)}(x)\right|<\infty ; \forall m, n \in \mathbb{N}\right\} .
$$

A norma em $H^{m}(\mathbb{R})$ é definida como

$$
\|f\|_{H^{m}}=\|f\|_{m}=\left(\int_{\mathbb{R}}\left(1+\xi^{2}\right)^{m}|\widehat{f}(\xi)|^{2} d \xi\right)^{1 / 2}
$$




\section{Introdução}

Consideramos a equação de Schrödringer não linear (NLS)

$$
i \partial_{t} u(x, t)+\partial_{x}^{2} u(x, t)+|u(x, t)|^{p} u(x, t)=0,
$$

onde $u: \mathbb{R} \times \mathbb{R} \longrightarrow \mathbb{C}$ e $0<p<\infty$. Na literatura atual, é bem conhecido que tal equação dispersiva descreve a evolução de um campo complexo em um meio de dispersão não linear (veja, por exemplo [2], [4], [5], [40] e suas referências). Portanto, a NLS desempenha um papel crucial em muitos campos de pesquisa, e abrange temas diversos tais como: óptica não linear, física de plasmas, dinâmica de fluidos e até mesmo modelos biofísicos .

Do ponto de vista matemático, o estudo da equação NLS (que inclui, por exemplo, a boa colocação local para o problema de valor inicial (PVI), o problema periódico de fronteira sob condições de regularidade mínimas sobre o dado inicial, o comportamento assintótico das soluções, ou bem o estudo da existência de "blow-up") tem crescido considerávelmente nos últimos anos. Neste contexto, a equação NLS, conhecida também como a equação de Gross-Pitaevskii (GP), possue certas extensões que a permitem ser usada para modelar, por exemplo, o condensado de Bose-Einstein (BEC) (ver [18]). O BEC é um estado da matéria que ocorre em determinados elementos quando a temperatura fica perto do zero absoluto (o conceito de zero absoluto é devido ao físico-matemático britânico William Thomson, e acontece quando um corpo não possui energia alguma, sendo que as suas moléculas ficam completamente paradas). Neste caso, o modelo a ser estudado tem a forma

$$
i \partial_{t} u+\partial_{x}^{2} u+V(x) u+|u|^{p} u=0
$$

onde o potencial $V(x)$ (fenômeno externo) é especificado dentro do contexto a ser pesquisado. Um tipo especifico de potêncial que surge no BEC (também em óptica não linear) é a equação de Schrödinger com uma interação de tipo delta (NLS- $\delta$ ),

$$
i \partial_{t} u+\partial_{x}^{2} u+Z \delta(x) u+|u|^{p} u=0
$$

onde $\delta$ é a distribuição delta de Dirac centrada na origem, a saber, $\langle\delta, v\rangle=v(0)$ para $v \in H^{1}(\mathbb{R})$ e $Z \in \mathbb{R}$. A equação (5), com $Z \neq 0$, tem sido também considerada em uma variedade ampla de modelos físicos com um ponto de defeito. De fato, nesta situação, a distribuição de Dirac é usada para modelar a aparição de uma impureza centrada na origem (veja [19], [20] e [35]). De maneira específica, a equação (5) está descrita pelo seguinte problema de fronteira (ver Caudrelier\&Mintchev\&Ragoucy [12])

$$
\left\{\begin{array}{l}
i \partial_{t} u(x, t)+\partial_{x}^{2} u(x, t)=-|u(x, t)|^{p} u(x, t), \quad x \neq 0 \\
\lim _{x \rightarrow 0^{+}}[u(x, t)-u(-x, t)]=0, \\
\lim _{x \rightarrow 0^{+}}\left[\partial_{x} u(x, t)-\partial_{x} u(-x, t)\right]=-Z u(0, t) \\
\lim _{x \rightarrow \pm \infty} u(x, t)=0 .
\end{array}\right.
$$


Portanto, a função $u(x, t)$ deve ser solução para a equação de Schrödinger não linear em $\mathbb{R}^{+}$ e $\mathbb{R}^{-}$, deve ser contínua em $x=0$ e satisfazer a condição de salto na derivada na origem.

Uma boa literatura tem sido desenvolvida para o modelo (5) nos últimos anos para os problemas de existência e estabilidade de ondas de tipo standing-wave, problemas de boa colocação, espalhamento e interação com solitons (ver [5], [16], [19], [20], [21], [22], [26], [27], [33], [35]).

A equação (5) pode também ser considerada como um modelo misto de interação, a saber; uma interação de um "wide-soliton"(sóliton amplo) com um potencial altamente localizado, neste caso sendo a distribuição delta centrada na origem (ver Holmer\&Marzuola\&Zworski [26]-[27]). O fenômeno de interação de solitons dispersivos com interações de tipo pontual já foi razoavelmente estudado. Em particular, em Holmer\&Zworski [28]- [29], para a equação (5) $\operatorname{com} p=2$, o dado inicial com perfil "travelling-wave"(onda viajante)

$$
u(x, 0)=e^{i c x} \operatorname{sech}\left(x-x_{0}\right), \quad x_{0}<<-1
$$

foi mostrado que para a $|Z|<<1$, a solução correspondente à onda viajante para $t>\left|x_{0}\right| / c$ permanece intacta. O caso $Z>0$ e $|c|>>1$ foi examinada em [26], [27] onde foi comprovado como o defeito separa o soliton em duas partes: uma parte é transmitida após o defeito, o outro é refletida no defeito. O caso $Z<0$ e $|c|>>1$ foi considerado em [16].

Outro tipo de potencial externo, $V(x)$, que foi estudado principalmente no contexto do BEC, é o denominado potencial de interação dupla. Potenciais de interação dupla constituem um particular interesse em óptica não linear: modelos de guias ópticas paralelas, munidas mediante uma canalização de feixes de luz com caudas evanescentes (condição dispersiva) (ver [2], [7], [11], [17], [25], [32], [36], [38], [39] e [22]). No contexto da óptica, um potencial duplo pode ser criado por um raio laser com duas "corcovas"em um meio de Kerr ([9]-[34]). Neste sentido, um potencial duplo clássico pode ser construído apartir da interação pontual de dois estados unitários (duas interações pontuais), desde que tais interações pontuais unitárias possuam um rápido decaimento. Noutras palavras, suponha que $V_{0}(x)$ representa um potencial unitário (centrado na origem) com decaimento rápido no infinito, e seja $L>0$; então, $V(x)=V_{0}(x+L)+V_{0}(x-L)$ representa um potencial duplo simétrico com decaimento rápido. Un clássico exemplo de um potencial simétrico duplo está dado pela seguinte família a dois parâmetros:

$$
V(x)=V(x ; \sigma, L)=\left[\frac{1}{\sqrt{4 \pi \sigma^{2}}} e^{-\frac{(x-L)}{4 \pi \sigma^{2}}}+\frac{1}{\sqrt{4 \pi \sigma^{2}}} e^{-\frac{(x+L)}{4 \pi \sigma^{2}}}\right] .
$$

Esta família de potenciais simétricos duplos tem a propriedade de que converge, quando $\sigma \rightarrow 0^{+}$, a uma dupla interação delta de Dirac centrada nos pontos $x= \pm L$, a saber: para $L>0$ fixo, temos, no sentido distribucional,

$$
V(x ; \sigma, L) \rightarrow \delta_{L}+\delta_{-L}, \quad \text { quando } \sigma \rightarrow 0^{+},
$$

onde $\delta_{ \pm L}$ representa a distribuição delta de Dirac em $x= \pm L$ : para qualquer $\phi$ na classe de Schwartz, $\left\langle\delta_{ \pm L}, \phi\right\rangle=\phi( \pm L)$.

Um dos principais objetivos deste trabalho é estudar a existência e estabilidade não-linear das soluções standing-waves (ondas estacionárias) associadas ao modelo (4), para $p=2$, quando o potencial, $V(x)$, é do tipo pontual duplo e simétrico, especificamente iremos a considerar o modelo (doravante NLS- $\delta_{ \pm c}$ )

$$
\left\{\begin{array}{l}
i \partial_{t} u(x, t)+\partial_{x}^{2} u(x, t)+Z\left[\delta_{c}(x)+\delta_{-c}(x)\right] u(x, t)=-|u(x, t)|^{2} u(x, t), \\
u(x, 0)=u_{0}(x)
\end{array}\right.
$$


com $u: \mathbb{R} \times \mathbb{R} \longrightarrow \mathbb{C}, Z \in \mathbb{R}$ e $\delta_{ \pm c}$ sendo as distribuições delta de Dirac centradas nos pontos $x= \pm c$. Concretamente, a equação $\left(\operatorname{NLS}-\delta_{ \pm c}\right)(7)$, com $Z \neq 0$, está descrita pelo seguinte problema de fronteira

$$
\left\{\begin{array}{l}
i \partial_{t} u(x, t)+\partial_{x}^{2} u(x, t)=-u(x, t)|u(x, t)|^{2}, \quad x \neq \pm c, \quad t \in \mathbb{R}, \\
\lim _{\varepsilon \rightarrow 0^{+}}[u( \pm c+\varepsilon, t)-u( \pm c-\varepsilon, t)]=0, \quad t \in \mathbb{R}, \\
\lim _{\varepsilon \rightarrow 0^{+}}\left[\partial_{x} u( \pm c+\varepsilon, t)-\partial_{x} u( \pm c-\varepsilon, t)\right]=-Z u( \pm c, t), \quad t \in \mathbb{R}, \\
\lim _{x \rightarrow \pm \infty} u(x, t)=0, \quad t \in \mathbb{R},
\end{array}\right.
$$

Portanto, $u(x, t)$ deve ser solução da equação de Schrödinger no conjunto $\mathbb{R}-\{ \pm c\}=$ $(-\infty,-c) \cup(-c, c) \cup(c, \infty)$; ser contínua em $x= \pm c$ e deve satifazer a condição de salto na derivada nos pontos $x= \pm c$.

A equação (7) foi estudada, por exemplo, por Jackson\&Weinstein em [30], sendo que o foco principal foi a análise da dinâmica das soluções "standing-waves". Essas soluções são da forma

$$
u(x, t)=e^{i \omega t} \Phi_{\omega, Z, c}(x)
$$

$\operatorname{para} \omega \in I \subset \mathbb{R}$ e

$$
\Phi_{\omega, Z, c} \in \mathcal{D}\left(-\frac{d^{2}}{d x^{2}}-Z\left(\delta_{c}+\delta_{-c}\right)\right) .
$$

Segue então que o perfil $\Phi \equiv \Phi_{\omega, Z, c}$ satisfaz o seguinte problema de fronteira,

$$
\left\{\begin{array}{l}
-\Phi^{\prime \prime}(x)+\omega \Phi(x)-\Phi^{3}(x)=0, \quad x \neq \pm c, \\
\Phi \in H^{1}(\mathbb{R}), \\
\lim _{\varepsilon \rightarrow 0^{+}}\left[\Phi^{\prime}( \pm c+\varepsilon)-\Phi^{\prime}( \pm c-\varepsilon)\right]=-Z \Phi( \pm c) .
\end{array}\right.
$$

Para $Z>0$, em [30], os autores mostram, via uma caracterização variacional, a existência de um perfil "standing-wave"para (10). Mais precisamente, mediante a minimização do funcional Hamiltoniano, $\mathcal{H}$,

$$
\mathcal{H}(\psi)=\int_{\mathbb{R}} \frac{1}{2}\left|\psi^{\prime}(x)\right|^{2}-\frac{1}{4}|\psi(x)|^{4} d x-\frac{1}{2} Z\left[|\psi(-c)|^{2}+|\psi(+c)|^{2}\right],
$$

sob a condição

$$
\mathcal{N}(\psi)=\int_{\mathbb{R}} \frac{1}{2}|\psi(x)|^{2} d x=\rho,
$$

onde $\rho>0$ é fixado. Tais minimizadores $\Phi$, chamados de ground states (estados fundamentais), satisfazem a equação de Euler-Lagrange determinada pela equação (10), onde a quantidade $\omega=\omega(\rho)$ será um multiplicador de Lagrange para o problema variacional determinado por (11) e (12). Já que $\omega(\rho)$ pertence a um intervalo conveniente, o problema variacional (11)-(12) dá origem a um ramo (família) de soluções estacionárias. Mais ainda, dado que as quantidades $\mathcal{H}$ e $\mathcal{N}$ são conservadas pelo fluxo do modelo (8), temos, via um argumento clássico dado por Cazenave\&Lions [13] e Cazenave [14], que o conjunto dos estados fundamentais (ground states set)

$$
\mathcal{G}_{\rho}=\left\{\Phi \in H^{1}(\mathbb{R}): \mathcal{H}(\Phi)=\min _{\psi} \mathcal{H}(\psi), \text { and } \mathcal{N}(\Phi)=\rho\right\}
$$

é não linearmente estável pelo fluxo gerado por (8) no espaço de energia $H^{1}(\mathbb{R})$. 
Notamos que um teorema geral para deduzir a existência e a estabilidade das ondas estacionárias para equações de Schrödinger unidimensionais com perturbações singulares do Laplaciano descrito por interações pontuais, foi recentemente estudado em Adami\&Noja\&Visciglia [1]. Embora vários resultados de estabilidade estabelecem que os "ground states" resultam ser dinamicamente estáveis, deixa sem resposta a questão de características qualitativas detalhadas do perfil da onda estacionária em (9). Como veremos abaixo, é possível demonstrar a existência de pelo menos duas famílias de soluções positivas para o problema de fronteira (10), com um perfil no intervalo [ $-c, c]$ dado pelas funções elípticas de Jacobi do tipo dnoidal e cnoidal.

Por outro lado, em [30], foi estabelecido um estudo global detalhado da quebra de simetria via métodos de bifurcação para o modelo (8) através de uma análise geométrica. A existência de um valor distinguido do parâmetro $c$ (the threshold-symmetric valor de $c$ ), foi estabelecido na Proposição 5 em [30], de tal modo que temos uma solução simétrica concentrada nos pontos de defeito, isto é, perfis que atingem os seus valores máximos nos dois pontos de defeito. Além disso, obteve-se um valor do parâmetro $c, c_{\text {thresh }}$, tal que para além deste valor limiar há um par de standing waves assimétricas do tipo cnoidal. Mais ainda, para $c \leqq c_{\text {thresh }}$, o estado simétrico (dnoidal-profile) são linearmente e não-linearmente instáveis, e para $c>c_{\text {thresh }}$, o estado assimétrico são não-linearmente orbitalmente estável.

Nosso objetivo neste trabalho será o de estabelecer a existência e estabilidade orbital de uma família de standing-wave para a NLS- $\delta_{ \pm c}$, onde o perfil $\Phi_{\omega, Z, c}$, será dado explicitamente pela fórmula

$$
\Phi_{\omega, Z, c}(x)=\left\{\begin{array}{llc}
\sqrt{2 \omega} \operatorname{sech}(\sqrt{\omega}(x+c-\gamma)), & \text { se } & -\infty<x<-c \\
\phi(x), & \text { se } & -c \leq x \leq c \\
\sqrt{2 \omega} \operatorname{sech}(\sqrt{\omega}(x-c+\gamma)), & \text { se } & c<x<\infty,
\end{array}\right.
$$

onde para $c$ fixado, $Z, \omega$ pertenecerão a intervalos específicos dependendo de $c$, aqui $\gamma$ é um parâmetro de translação dependendo de $Z, \omega$. A função $\phi$ irá ter um perfil de tipo dnoidal ou cnoidal para os casos de $Z>0$ ou $Z<0$, respectivamente (ver Teoremas 3.6 e 3.10 abaixo). Além disso, o perfil $\phi$ possuirá a propriedade de ser côncava no intervalo $[-c, c]$. Ainda mais, nossa construção do peak-standing waves $\Phi_{\omega, Z, c}$ em (14) garante a sua convergência na $H^{1}(\mathbb{R})$-norma para o soliton clássico da $\operatorname{NLS}(Z=0$ em (1)), quando o parâmetro $Z$ vai para 0 . Este fato será crucial para estudar o índice de Morse associado a um operador auto-adjunto específico (ver (1.4) abaixo), que será essencial para a nossa teoria de estabilidade.

Agora, a simetria básica associada à equação (8) é a invariância por rotação, ou seja, se $u(x, t)$ é solução de $(8)$, então $V(x, t) \equiv e^{i \theta} u(x, t)$ também é solução para (8) para cada $\theta \in[0,2 \pi]$. A simetria de translação não se aplica neste caso devido à presença dos pontos de defeito. Assim, a nossa noção de estabilidade e instabilidade de soluções de tipo onda estacionária é formulada da seguinte forma:

Definição 0.1 Para $\eta>0$, consideremos $\Phi_{\omega}=\Phi_{\omega, Z, c}$ e definimos

$$
\mathcal{U}_{\eta}\left(\Phi_{\omega}\right)=\left\{v \in H^{1}(\mathbb{R}): \inf _{\theta \in \mathbb{R}}\left\|v-e^{i \theta} \Phi_{\omega}\right\|_{H^{1}}<\eta\right\}
$$

Dizemos que uma onda estacionária $e^{i \omega t} \Phi_{\omega}$ de (8) é orbitalmente estável em $H^{1}(\mathbb{R})$ pelo fluxo determinado por (8), se para qualquer $\varepsilon>0$ existe $\eta>0$ tal que para qualquer $u_{0} \in \mathcal{U}_{\eta}\left(\Phi_{\omega}\right)$, a solução $u(t)$ de $(8)$, com $u(0)=u_{0}$, satisfaz $u(t) \in \mathcal{U}_{\varepsilon}\left(\Phi_{\omega}\right)$, para qualquer $t \geq 0$. Caso contrário, $e^{i \omega t} \Phi_{\omega}$ é dita ser orbitalmente instável em $H^{1}(\mathbb{R})$. 
Nossa abordagem para a teoria de estabilidade em $H^{1}(\mathbb{R})$ da família peak-standing waves $\Phi_{\omega, Z, c}$ em (14), será baseado na teoria geral de estabilidade desenvolvida por Grillakis\&Shatah\& Strauss [23], [24], para um sistema Hamiltoniano que é invariante sob um grupo unitário a um parâmetro de operadores. Assim, nosso principal resultado de estabilidade é o seguinte:

Teorema 0.2 Seja $c>0$. Para $\omega$ como nos Teoremas 3.6 e 3.10 abaixo, a saber, para $Z \in\left(0, \frac{\pi}{4 c}\right)$ nós temos $\omega \in\left(\frac{1}{c^{2}}\left[\operatorname{sech}^{-1}\left(\frac{1}{\sqrt{2}}\right)\right]^{2}, \frac{\pi^{2}}{8 c^{2}}\right)$ e para $Z \in(-\infty, 0)$ escolhemos $\omega \in$ $\left(0, \frac{1}{c^{2}}\left[\operatorname{sech}^{-1}\left(\frac{1}{\sqrt{2}}\right)\right]^{2}\right)$. Consideremos a familia de peak-standing waves $\Phi_{\omega, Z, c}$ em (14) (ver (3.33) e (3.45) abaixo). Então, tem-se:

1. Para $Z>0$, a peak-standing wave de tipo dnoidal $e^{i \omega t} \Phi_{\omega, Z, c}$ é instável em $H^{1}(\mathbb{R})$ pelo fluxo determinado pela $N L S-\delta_{ \pm c}(7)$.

2. Para $Z<0$, a peak-standing wave de tipo cnoidal $e^{i \omega t} \tilde{\Phi}_{\omega, Z, c}$ é estável em $H^{1}(\mathbb{R})$ pelo fluxo determinado pela $N L S-\delta_{ \pm c}(7)$.

3. Para $Z>0$, a peak-standing wave de tipo dnoidal $e^{i \omega t} \Phi_{\omega, Z, c}$ é estável em $H_{\text {par }}^{1}(\mathbb{R})$ pelo fluxo deteminado pela $N L S-\delta_{ \pm c}(7)$.

Na figura 3.8 abaixo, mostramos o correspondente diagrama de fase em coordenadas $\left(\Phi, \Phi^{\prime}\right)$, associado ao caso $Z>0$, ou seja o perfil de tipo dnoidal $\Phi \equiv \Phi_{\omega, Z, c}$ dado no Teorema 3.6. Na condição de salto na derivada nos pontos $x= \pm c$ (representada pela reta vertical), consideramos o caso $\omega=2$, e $c=0,63 ; 0,64$ e 0,66 . Note que a trajetória da "parte dnoidal"da $\Phi$ está no interior da separatrix determinada pelo soliton "secante hiperbólica", e é percorrida no sentido horário- como indicam as setas- (concave profile on $[-c, c]$ ). A diferença das trajetórias do nosso trabalho com aquele estudado por Jackson\&Weinstein em [30], é que estas últimas estão no sentido anti-horário (convex profile on $[-c, c]$ ). Assim do Teorema 0.2 acima e dos resultados em Jackson\&Weinstein podemos concluir que as standing-wave de tipo peakon com um perfil dnoidal em $[-c, c]$ são orbitalmente instáveis.

Agora, na figura 3.11 abaixo mostramos o caso $Z<0$, ou seja o perfil de tipo cnoidal $\zeta \equiv \Phi_{\omega, Z, c}$ dado no Teorema 3.10. Este diagrama, em coordenadas $\left(\zeta, \zeta^{\prime}\right)$ é para o caso particular em que $\omega=2$, e $c=0,63 ; 0.64$ e 0,65. Notamos que a trajetória da parte "cnoidal" deste diagrama, a qual está no exterior da separatrix determinada pelo soliton, é percorrida também no sentido horário. O caso de considerar trajetórias cnoidais no sentido anti-horário serão os objetivos de futuros estudos.

A prova do Teorema 0.2 será feita no Capítulo 4. Para este, o uso da teoria de Floquet, resultados sobre a representação de formas bilineares, teoria de extensão para operadores simétricos (teorias de Klein e von Neumann), e da teoria de perturbação analítica de operadores serão ferramentas essenciais em nosso analise. O uso dos projetores de Riesz serão necessarios em um argumento de continuação para o estudo do índice de Morse de um específico operador auto-adjunto. O uso de ferramentas de tipo computacional serão de muita utilidade em nosso análise de estabilidade.

Descreveremos brevemente os conteúdos de cada capítulo deste trabalho. No Capítulo 1, apresentamos os preliminares e uma breve revisão de resultados que utilizaremos no nosso trabalho. No Capítulo 2, estudamos a boa colocação local e global do $(7)$ em $H^{1}(\mathbb{R})$. No Capítulo 3, estabelecemos a teoria de existência de soluções de tipo peak-standing waves satisfazendo (8). O uso da teoria de funções elípticas de Jacobi será de fundamental importância. No Capítulo 4, estabelecemos nossa teoria de estabilidade para os perfiles do tipo dado em (14). O uso da teoria de perturbação analítica e da teoria de Floquet serão as ferramentas básicas para este estudo. No Capítulo 5 estabelecemos um conjunto de propostas de 
futuros trabalhos, assim como, o andamento de alguns específicos problemas. No Apêndice apresentaremos alguns cálculos relevantes que forám usados no decorrer dos capítulos desta tese. 


\section{Capítulo 1}

\section{Preliminares}

Neste capítulo faremos uma breve revisão dos principais resultados que utilizaremos para o desenvolvimento do nosso trabalho. Tais resultados, que incluem definições, teoremas e proposições; podem ser consultados nas referências bibliográficas desta tese. Começaremos dando a definição de estabilidade orbital no espaço $H^{1}(\mathbb{R})$. Em seguida, trataremos sobre o marco fundamental no qual baseamos os resultados obtidos, a saber; os trabalhos desenvolvidos por Grillakis\&Shatah\&Strauss em [23] e [24]. De maneira precisa, damos as condições suficientes nas quais é possível obter resultados de estabilidade/instabilidade orbital em $H^{1}(\mathbb{R})$. Finalmente, trataremos sobre os resultados clássicos de boa colocação local em $H^{1}(\mathbb{R})$ (Cazenave [14]), bem como de algumas propriedades básicas das funções elípticas de Jacobi, as quais constituem nossa principal ferramenta para estabelecer um perfil periódico entre os pontos de interação, $c$ e $-c$, na equação (8).

\subsection{Estabilidade Orbital}

Concretamente, o principal objetivo desta tese consiste em estabelecer a existência e estabilidade orbital para uma família de soluções do tipo standing-wave, $e^{i \omega t} \Phi_{\omega, Z, c}$, associadas à equação NLS- $\delta_{ \pm c}(\operatorname{ver}(7))$, sendo que a função $\Phi_{\omega, Z, c}$ está dada explícitamente pela fórmula

$$
\Phi_{\omega, Z, c}(x)=\left\{\begin{array}{llc}
\sqrt{2 \omega} \operatorname{sech}(\sqrt{\omega}(x+c-\gamma)), & \text { se } & -\infty<x<-c \\
\phi(x), & \text { se } & -c \leq x \leq c \\
\sqrt{2 \omega} \operatorname{sech}(\sqrt{\omega}(x-c+\gamma)), & \text { se } & c<x<\infty,
\end{array}\right.
$$

onde $c>0$ é fixado e $Z, \omega$ pertencem a intervalos específicos, os quais dependem de $c$. Aqui, $\gamma$ representa uma translação (depende de $Z$ e $\omega$ ); e $\phi$ coincide com um perfil do tipo dnoidal ou cnoidal para os casos $Z>0$ e $Z<0$, respectivamente.

A simetria básica que possui o modelo NLS- $\delta_{ \pm c}$ é a simetria por rotações, isto significa que se $u(x, t)$ é uma solução de (7), então, para todo $\theta \in[0,2 \pi], v(x, t)=e^{i \theta} u(x, t)$ também é uma solução de (7). A simetria por translações não é obtida devido à presença das interações delta no modelo. Assim, a definição de estabilidade orbital para as soluções standing-wave associadas a este modelo vem dada como segue,

Definição 1.1 Para $\eta>0$, seja $\Phi_{\omega}=\Phi_{\omega, Z, c}$ e defina

$$
\mathcal{U}_{\eta}\left(\Phi_{\omega}\right)=\left\{v \in H^{1}(\mathbb{R}): \inf _{\theta \in \mathbb{R}}\left\|v-e^{i \theta} \Phi_{\omega}\right\|_{H^{1}}<\eta\right\}
$$

Diz-se que a solução standing-wave, $e^{i \omega t} \Phi_{\omega}$, de (7) é orbitalmente estável em $H^{1}(\mathbb{R})$ pelo fluxo determinado por (7), se para qualquer $\varepsilon>0$ existe $\eta>0$, com a propriedade de que 
para todo $u_{0} \in \mathcal{U}_{\eta}\left(\Phi_{\omega}\right)$, a solução $u(t)$ of $(7)$, com $u(0)=u_{0}$, satisfaz que $u(t) \in \mathcal{U}_{\varepsilon}\left(\Phi_{\omega}\right)$, para todo $t \geq 0$. Caso contrário, $e^{i \omega t} \Phi_{\omega}$ é dita orbitalmente instável em $H^{1}(\mathbb{R})$.

A teoria de estabilidade orbital em $H^{1}(\mathbb{R})$ da família "peak-standing-wave"dada em (1.1), está baseada nos trabalhos desenvolvidos por Grillakis\&Shatah\&Strauss em [23] e [24] para um sistema Hamiltoniano que é invariante sob a ação de um grupo unitário de operadores contendo um parâmetro. Considere o domínio

$$
\mathcal{D}=\left\{f \in H^{1}(\mathbb{R}) \cap H^{2}(\mathbb{R} \backslash\{ \pm c\}): f^{\prime}\left( \pm c^{+}\right)-f^{\prime}\left( \pm c^{-}\right)=-Z f( \pm c)\right\},
$$

então

- O problema de Cauchy: o problema de valor inicial (PVI) associado à equação NLS- $\delta_{ \pm c}$, (7), é globalmente bem posto em $H^{1}(\mathbb{R})$.

- A condição espectral:

(a) O operador auto adjunto $\mathcal{L}_{2, Z, c}$, definido em $L^{2}(\mathbb{R})$ por

$$
\mathcal{L}_{2, Z, c}=-\frac{d^{2}}{d x^{2}}+\omega-\Phi_{\omega, Z, c}^{2}
$$

e cujo domínio é $\mathcal{D}$, é um operador não-negativo. Além disso, o zero é um autovalor simples cuja auto-função associada é $\Phi_{\omega, Z, c}$.

(b) O operador auto-adjunto $\mathcal{L}_{1, Z, c}$, definido em $L^{2}(\mathbb{R})$ por

$$
\mathcal{L}_{1, Z, c}=-\frac{d^{2}}{d x^{2}}+\omega-3 \Phi_{\omega, Z, c}^{2}
$$

com domínio $\mathcal{D}$, tem núcleo trivial para todo $Z \neq 0$ admissível. Como veremos no trabalho, o sinal de $Z$ faz com que este parâmetro pertença a um determinado intervalo da reta. Esta é a razão para falar de valores admissíveis de $Z$.

(c) O número de auto-valores negativos de $\mathcal{L}_{1, Z, c}$.

- A slope condition: O sinal da quantidade $\partial_{\omega}\left\|\Phi_{\omega, Z, c}\right\|_{2}^{2}$.

No Capítulo 4 faremos uma análise detalhada destes operadores, a qual inclui a maneira como eles aparecem na teoria de estabilidade. Da análise dos operadores $\mathcal{L}_{1, Z, c}$ e $\mathcal{L}_{2, Z, c}$ definidos anteriormente, temos o seguinte teorema,

Teorema 1.2 [Grillakis\&ShatahछStrauss and Weinstein] Suponha que $\operatorname{ker}\left(\mathcal{L}_{2, Z, c}\right)=\left[\Phi_{\omega_{0}, Z, c}\right]$ $e \operatorname{ker}\left(\mathcal{L}_{1, z, c}\right)=\{0\}$. Então:

1. O peak standing-wave, $e^{i \omega_{0} t} \Phi_{\omega_{0}, Z, c}$, é orbitalmente estável em $H^{1}(\mathbb{R})$ se

$$
n\left(\mathcal{H}_{\omega_{0}, Z, c}\right)=p\left(\omega_{0}\right) .
$$

2. O peak standing-wave, $e^{i \omega_{0} t} \Phi_{\omega_{0}, Z, c}$, é orbitalmente instável em $H^{1}(\mathbb{R})$ se

$$
n\left(\mathcal{H}_{\omega_{0}, Z, c}\right)-p\left(\omega_{0}\right) \text { é ímpar. }
$$

Neste caso,

$$
p\left(\omega_{0}\right)=\left\{\begin{array}{l}
1, \text { se } \partial_{\omega}\left\|\Phi_{\omega, Z, c}\right\|_{2}^{2}>0 \text { em } \omega=\omega_{0} \\
0, \text { se } \partial_{\omega}\left\|\Phi_{\omega, Z, c}\right\|_{2}^{2}<0 \text { em } \omega=\omega_{0}
\end{array}\right.
$$

$e$

$$
\mathcal{H}_{\omega, Z, c}=\left(\begin{array}{cc}
\mathcal{L}_{1, Z, c} & 0 \\
0 & \mathcal{L}_{2, Z, c}
\end{array}\right) .
$$

Demonstração. Ver [23] e [24] 


\subsection{Boa colocação do problema de Cauchy}

Um dos requisitos necessários para o resultado de estabilidade/instabilidade é a boa colocação global do (PVI) NLS- $\delta_{ \pm c},(7)$, no espaço $H^{1}(\mathbb{R})$. Introduzimos a seguir o conceito de boa colocação local/global.

Definição 1.3 Sejam $X, Y$ espaços de Banach, $T_{0} \in(0, \infty)$ e seja $F:\left[0, T_{0}\right] \times Y \longrightarrow X$ uma função contínua. Dizemos que o problema de Cauchy

$$
\left\{\begin{array}{l}
\partial_{t} u(t)=F(t, u(t)) \in X, \\
u(0)=\phi \in Y
\end{array}\right.
$$

\section{é localmente bem posto em Y se}

1. Existe $T \in\left[0, T_{0}\right)$ e uma função $u \in C([0, T], Y)$ tal que $u(0)=\phi$ e a equação diferencial em (1.5) é satisfeita no sentido

$$
\lim _{h \rightarrow 0}\left\|\frac{u(t+h)-u(t)}{h}-F(t, u(t))\right\|_{X}=0 .
$$

Aqui, as derivadas em $t=0$ e $t=T$ são calculadas pela direita e pela esquerda, respectivamente. (Existência)

2. O problema (1.5) tem, no máximo, uma solução em $C([0, T] ; Y)$. (Unicidade)

3. A aplicação $\phi \rightarrow u$ é continua. Mais precisamente, sejam $\phi_{n} \in Y, n \in \mathbb{N}$, tal que $\phi_{n} \rightarrow$ $\phi_{\infty}$ na topologia de $Y$, e sejam $u_{n} \in C\left(\left[0, T_{n}\right] ; Y\right)$ as soluções correspondentes. Seja $T \in\left[0, T_{\infty}\right)$. Então as soluções $u_{n}$ estão definidas em $[0, T]$, para $n$ suficientemente grande, $e$

$$
\lim _{n \rightarrow \infty} \sup _{[0, T]}\left\|u_{n}(t)-u_{\infty}(t)\right\|_{Y}=0 \text {. (Dependência contínua do dado inicial) }
$$

Se alguma destas três condições não for satisfeita, dizemos que o problema (1.5) é localmente mau colocado em $Y$. Se as três condições anteriores são verificadas para todo $t \in[0, \infty)$, dizemos que o problema (1.5) é globalmente bem posto em $Y$.

Em primeiro lugar, resolvemos o problema da boa colocação local em $H^{1}(\mathbb{R})$. A prova disto está apoiada, fundamentalmente, em dois fatos: um deles é o seguinte

Teorema 1.4 Seja $X$ um espaço de Hilbert complexo com produto interno $(\cdot, \cdot)_{X}$. Seja $\mathcal{A}$ um operador lineal auto-adjunto, com $\mathcal{A} \leq 0$-ou seja $-\mathcal{A}$ é positivo-, e cujo domínio é $\mathcal{D}(\mathcal{A})$.

Denote por $X_{\mathcal{A}}$ o completamento de $\mathcal{D}(\mathcal{A})$, ou seja, tal que $\overline{X_{\mathcal{A}}}=X$, associado à norma

$$
\|x\|_{X_{\mathcal{A}}}^{2}=\|x\|_{X}^{2}-(\mathcal{A} x, x)_{X} .
$$

Sejam também $X_{\mathcal{A}}^{*}=\left(X_{A}\right)^{*}$ o espaço dual de $X_{\mathcal{A}}$ e $\overline{\mathcal{A}}$ a extensão de $\mathcal{A}$ ao conjunto $(\mathcal{D}(\mathcal{A}))^{*}$. Finalmente, seja $\tau(t)$ o grupo de isometrias gerado em $(\mathcal{D}(\mathcal{A}))^{*}, X_{\mathcal{A}}^{*}, X, X_{\mathcal{A}}$ ou $\mathcal{D}(\mathcal{A})$ pelo operador "skew-adjoint" $i \mathcal{A}$. Assuma que $g: X \longrightarrow X$ é Lipschitz contínua nos conjuntos 
limitados de $X$, e que existe $G \in C^{1}\left(X_{\mathcal{A}} ; \mathbb{R}\right)$ tal que $G^{\prime}(x)=g(x)$ para todo $x \in X_{\mathcal{A}}$. Assuma também que

$$
(g(x), i x)_{X}=0, \text { para } x \in X .
$$

Para $x \in X_{\mathcal{A}}$, seja

$$
E(x)=\frac{1}{2}\left(\|x\|_{X_{\mathcal{A}}}^{2}-\|x\|_{X}^{2}\right)-G(x)=-\frac{1}{2}(\mathcal{A} x, x)_{X}-G(x),
$$

então $E \in C^{1}\left(X_{\mathcal{A}} ; \mathbb{R}\right) e$

$$
E^{\prime}(x)=\mathcal{A} x-g(x) \in X_{\mathcal{A}}^{*}, \text { para cada } x \in X_{\mathcal{A}}^{*} .
$$

Segue então que, para cada $x \in X$, existe uma única solução u do problema

$$
\left\{\begin{array}{l}
u \in \mathcal{C}(\mathbb{R} ; X) \cap C^{1}\left((\mathcal{D}(\mathcal{A}))^{*}\right), \\
i \frac{d u}{d t}+\overline{\mathcal{A}} u+g(u)=0, \text { para todo } t \in \mathbb{R} \\
u(0)=x
\end{array}\right.
$$

Além disto, valem as seguintes propriedades:

1. $\|u(t)\|_{X}=\|x\|_{X}, \forall t \in \mathbb{R} \quad$ (conservação da carga);

2. Se $x \in X_{\mathcal{A}}$, então $u \in C\left(\mathbb{R} ; X_{\mathcal{A}}\right) \cap C^{1}\left(\mathbb{R} ; X_{\mathcal{A}}^{*}\right)$ e $E(u(t))=E(x)$ (conservação $d a$ energia);

3. Se $x \in \mathcal{D}(\mathcal{A})$, então $u \in C(\mathbb{R} ; \mathcal{D}(\mathcal{A})) \cap C^{1}(\mathbb{R} ; X)$.

Demonstração. Ver [14, Teorema 3.3.1] e [14, Teorema 3.7.1] .

\subsection{Interações pontuais}

Um outro fato que usaremos em nossa teoria de boa colocação é aquele que envolve o espectro do operador

$$
-\Delta_{ \pm c, Z}=-\frac{d^{2}}{d x^{2}}-Z\left[\delta_{c}+\delta_{c}\right]
$$

Esta expressão é entendida como sendo uma família de extensões auto-adjuntas asociadas ao operador simétrico

$$
\left\{\begin{array}{l}
-\Delta=-\frac{d^{2}}{d x^{2}} \\
D(-\Delta)=\left\{f \in H^{1}(\mathbb{R}) \cap H^{2}(\mathbb{R}-\{ \pm c\}): f( \pm c)=0\right\} .
\end{array}\right.
$$

De maneira geral, iremos a considerar o operador de Schrödinger com um número finito de $\delta$-interações: Sejam $\gamma_{1}, \gamma_{2}, \ldots, \gamma_{N} \in \mathbb{R} \backslash\{0\}$, e $x_{1}, x_{2}, \ldots, x_{N} \in \mathbb{R} ;$ com $N \in \mathbb{N}$. Sejam também $\gamma=\left\{-\gamma_{j}\right\}_{j=1}^{N}$ e $X=\left\{x_{j}\right\}_{j=1}^{N}$. O operador de Schrödinger com $N$ interações de tipo delta de Dirac, está formalmente definido como

$$
l_{\gamma, X}=-\frac{d^{2}}{d x^{2}}-\sum_{j=1}^{N} \gamma_{j} \delta\left(x-x_{j}\right)
$$


definido em $L^{2}(\mathbb{R})$, e com domínio

$$
D\left(l_{\gamma, X}\right)=\left\{f \in H^{1}(\mathbb{R}) \cap H^{2}(\mathbb{R} \backslash X): f^{\prime}\left(x_{j}^{+}\right)-f^{\prime}\left(x_{j}^{-}\right)=-\gamma_{j} f\left(x_{j}\right)\right\} .
$$

A notação $l_{\gamma, X}$ é usada para indicar que a interação delta ocorre nos $N$ pontos do conjunto $X=\left\{x_{1}, x_{2}, \ldots, x_{N}\right\}$; e tal que, para cada ponto $x_{j} \in X(j=1,2, \ldots, N)$ vale a condição de salto na derivada $f^{\prime}\left(x_{j}^{+}\right)-f^{\prime}\left(x_{j}^{-}\right)=-\gamma_{j} f\left(x_{j}\right)$; para $-\gamma_{j} \in \gamma$. De forma concreta, este operador pode ser entendido da seguinte maneira: Considere o operador $A=-\frac{d^{2}}{d x^{2}}$ sobre $L^{2}(\mathbb{R})$, com domínio $\mathcal{D}(A)=H^{2}(\mathbb{R})$, e sua respectiva restrição

$$
\left\{\begin{array}{l}
A_{0}=\left.A\right|_{D\left(A_{0}\right)} ; \\
D\left(A_{0}\right) \equiv\left\{g \in \mathcal{D}(A): g\left(x_{j}\right)=0\right\}, \quad j=1,2, . ., N .
\end{array}\right.
$$

O adjunto de $A_{0}, A_{0}^{*}$, vem dado por (ver Alveberio et al. em [3, seção I.3.1])

$$
\left\{\begin{array}{l}
A_{0}^{*}=-\frac{d^{2}}{d x^{2}}, \\
D\left(A_{0}^{*}\right)=D\left(l_{\gamma, X}\right) .
\end{array}\right.
$$

Note que a delta-interação no ponto $x_{j}$, denotada por $\delta_{j}$, é um funcional linear no espaço $H^{1}(\mathbb{R})$, pois $\left|\left\langle\delta_{j}, \varphi\right\rangle\right|=\left|\varphi\left(x_{j}\right)\right| \leq c|| \varphi \|_{H^{1}}$, para todo $\varphi \in H^{1}(\mathbb{R})$. Assim, para $\xi \in H^{1}(\mathbb{R})$, temos o operador $l_{\gamma, X}$

$$
\left\{\begin{array}{l}
l_{\gamma, X}=-\frac{d^{2}}{d x^{2}} \xi(x), \quad x \in \mathbb{R} \backslash X \\
\xi^{\prime}\left(x_{j}\right)-\xi^{\prime}\left(x_{j}\right)=-\gamma_{j} \xi\left(x_{j}\right) .
\end{array}\right.
$$

Com a notação acima, temos o seguinte

Teorema 1.5 Sejam $\gamma$ e X como definidos anteriormente. Então,

1. Todos os autovalores de $l_{\gamma, X}$ são negativos e simples. Além disso, se $n\left(l_{\gamma, X}\right)$ representa o número de autovalores negativos de $l_{\gamma, X}$, então $n\left(l_{\gamma, X}\right) \leq N$. Seja $\sigma_{p}$ o espectro pontual de $l_{\gamma, X}$, então $k^{2} \in \sigma_{p} \cap(-\infty, 0) \Leftrightarrow \operatorname{det}\left(\Gamma_{\gamma, X}(k)\right)=0$. Aqui

$$
\Gamma_{\gamma, X}(k)=\left(\frac{1}{\gamma_{j}} \delta_{j l}-G_{k}\left(x_{j}-x_{l}\right)\right)_{j, l=1}^{N},
$$

e $\delta_{j l}$ é a delta de Kronecker. A função $G_{k}$ está definida por

$$
G_{k}\left(x-x_{j}\right)=\frac{i}{2 k} e^{i k\left|x-x_{j}\right|}, \operatorname{Im}(k)>0 .
$$

2. Se $E_{0}=k_{0}^{2}$ pertence a $\sigma_{p}$, então a autofunção associada a $E_{0}$ está dada por

$$
\Psi_{E_{0}}(x)=\sum_{j=1}^{N} c_{j} G_{k_{0}}\left(x-x_{j}\right),
$$

onde $\left(c_{1}, c_{2}, \ldots c_{N}\right)^{T}$ é o autovetor da matriz $\Gamma_{\gamma, X}\left(k_{0}\right)$.

3. O resto do espectro de $l_{\gamma, X}$ é absolutamente continuo e positivo, isto é, $\sigma_{\text {ess }}\left(l_{\gamma, X}\right)=$ $\sigma_{\text {abs. cont. }}\left(l_{\gamma, X}\right)=\mathbb{R}^{+}$. 
Demonstração. Ver Alveberio et al. [3, seção 2.1.3, capítulo II.2]

No capítulo 2 mostraremos como usar o Teorema 1.5 no caso particular em que $\left(x_{1}, x_{2}\right)=$ $(c,-c)$ e $\left(\gamma_{1}, \gamma_{2}\right)=(Z,-Z)$, ou seja, no caso em que $\gamma=\{Z,-Z\}$ e $X=\{c,-c\}$, para provar a boa coloção local em $H^{1}(\mathbb{R})$. De fato, usando o Teorema 1.5, definiremos uma norma equivalente à norma usual em $H^{1}(\mathbb{R}) \mathrm{e}$, em virtude do Teorema 1.4, poderemos provar a boa colocação local em $H^{1}(\mathbb{R})$ para o (PVI) associado à equação (7). Já a boa colocação global do (PVI), seguirá como consequência das quantidades conservadas do modelo (7) (carga e energia, dadas no Teorema 1.4).

Para finalizar este capítulo trateremos de forma rápida a maneira em que aparecem as denominadas funções elípticas de Jacobi, bem como as suas propriedades mais importantes. Para um estudo mais abrangente destes conceitos veja Byrd\&Friedman em [10].

\subsection{Funções elípticas de Jacobi}

Para $-1 \leq y_{1} \leq 1$ e $k \in(0,1)$, considere a função $u$ dada pela fórmula

$$
u\left(y_{1} ; k\right) \equiv u \equiv \int_{0}^{y_{1}} \frac{d t}{\sqrt{\left(1-t^{2}\right)\left(1-k^{2} t^{2}\right)}}=\int_{0}^{\varphi} \frac{d \theta}{\sqrt{1-k^{2} \operatorname{sen}^{2} \theta}}=F(\varphi ; k)
$$

com $y_{1}=\operatorname{sen}(\varphi)$. A função $u$ é uma integral elíptica clássica, chamada a integral elíptica de primeiro tipo. Este tipo de integral aparece de forma natural no estudo da dinâmica de um pêndulo ideal (aquele que suspende um corpo através de um fio inextensível cujo peso é desprezível). Abel e Jacobi foram os primeiros matemáticos interessados no problema de inverter este tipo de integral. Tal problema é análogo ao problema que surge na definição das funções trigonomêtricas inversas, por exemplo; $y=\operatorname{sen}(u)$ define a inversa da integral

$$
u=\int_{0}^{y} \frac{d t}{\sqrt{1-t^{2}}}, \quad(|y| \leq 1)
$$

De fato, uma das funções elípticas de Jacobi aparece do processo de inversão da integral (1.6). É a conhecida função snoidal. Desta maneira, $y_{1}=\operatorname{sen}(\varphi)=\operatorname{sn}(u ; k)$, ou simplesmente $y_{1}=\operatorname{sn}(u)$, quando não for necessário explicitar o número $k$; e $\varphi=a m(u ; k)$. A função $s n(u)$ é uma função elíptica ímpar de ordem dois, é periódica e de classe $C^{\infty}(\mathbb{R})$. Da função snoidal é possível definir outras duas funções de classe $C^{\infty}(\mathbb{R})$; a saber: a função cnoidal definida por

$$
c n(u ; k)=\sqrt{1-y_{1}^{2}}=\cos (\varphi),
$$

e a função dnoidal, definida por

$$
d n(u ; k)=\sqrt{1-k^{2} y_{1}^{2}}=\sqrt{1-k^{2} \operatorname{sen}^{2} \varphi} .
$$

As funções snoidal, cnoidal e dnoidal têm períodos fundamentais reais iguais a $4 K(k) ; 4 K(k)$ e $2 K(k)$, respectivamente; onde

$$
K(k)=F\left(\frac{\pi}{2} ; k\right)=\int_{0}^{1} \frac{d t}{\sqrt{\left(1-t^{2}\right)\left(1-k^{2} t^{2}\right)}}
$$


e é chamada de integral elíptica completa de primeiro tipo. Tais funções verificam as identidades básicas: $s n^{2}(u)+c n^{2}(u)=1, k^{2} s n^{2}(u)+d n^{2}(u)=1, \operatorname{sn}(u, 0)=\operatorname{sen}(u)$, $c n(u, 0)=\cos (u), \operatorname{sn}(u, 1)=\tanh (u)$ e $\operatorname{cn}(u, 1)=d n(u, 1)=\operatorname{sech}(u)$. As derivadas das funções elípticas de Jacobi snoidal, cnoidal e dnoidal são, para $\gamma \in \mathbb{R}$,

$$
\left\{\begin{array}{l}
\frac{d s n(\gamma)}{d \gamma}=c n(\gamma) d n(\gamma), \\
\frac{d c n(\gamma)}{d \gamma}=-\operatorname{sn}(\gamma) d n(\gamma), \\
\frac{d d n(\gamma)}{d \gamma}=-k^{2} \operatorname{sn}(\gamma) c n(\gamma)
\end{array}\right.
$$

A integral elíptica de segundo tipo está definida por

$$
\begin{aligned}
\int_{0}^{y_{1}} \sqrt{\frac{1-k^{2} t^{2}}{1-t^{2}}} d t & =\int_{0}^{\varphi} \sqrt{1-k^{2} \operatorname{sen}^{2} \theta} d \theta=\int_{0}^{u_{1}} d n^{2}(u) d u \\
& =E\left(u_{1}\right) \equiv E\left(\operatorname{am} u_{1} ; k\right) \equiv E(\varphi ; k),
\end{aligned}
$$

onde $y_{1}=\operatorname{sen}(\varphi)$, é como na integral (1.6). Este tipo de integral será crucial para determinar a convexidade de uma certa função em nosso resultado de estabilidade/instabilidade orbital. Aqui,

$$
E(k)=E\left(\frac{\pi}{2} ; k\right)=\int_{0}^{1} \sqrt{\frac{1-k^{2} t^{2}}{1-t^{2}}} d t .
$$

é a integral elíptica completa de segundo tipo. Usando programa Wolfram-Mathematica 9.0, mostramos abaixo os gráficos de $K(k)$ e $E(k)$, para $k \in(0,1)$.

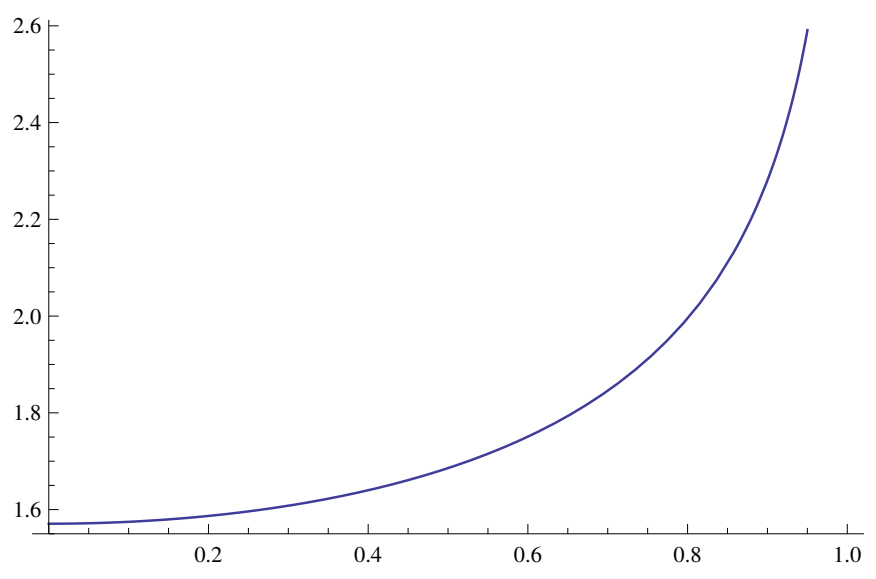

Figura 1.1: Gráfico da função $K(k)$

A função Zeta de Jacobi, $Z(u)=Z(u, k)$, é definida por

$$
Z\left(u_{1}\right)=\int_{0}^{u_{1}}\left[d n^{2}(u)-\frac{E(k)}{K(k)}\right] d u .
$$




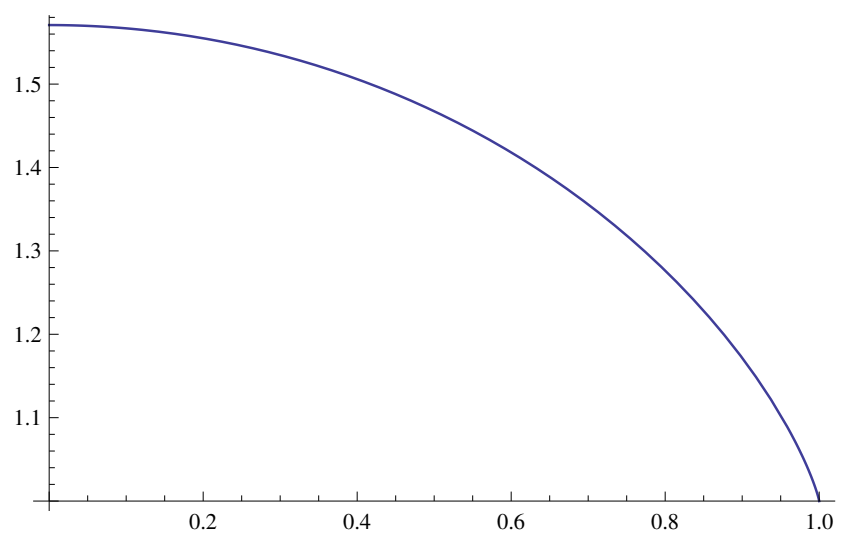

Figura 1.2: Gráfico da função $E(k)$

Esta função é impar, periódica de período fundamental $2 K(k)$. Além disso, $Z(\pi / 2, k)=0$ e $Z(m K)=0$, para $m=0,1,2, \cdots$.

Em nosso análise iremos também usar as seguintes funções inversas de Jacobi,

$$
\begin{array}{ll}
c n^{-1}(y ; k)=\int_{y}^{1} \frac{d t}{\sqrt{\left(1-t^{2}\right)\left(k^{2}+k^{2} t^{2}\right)}}, & 0 \leqq y<1, \\
d n^{-1}(y ; k)=\int_{y}^{1} \frac{d t}{\sqrt{\left(1-t^{2}\right)\left(t^{2}-k^{\prime 2}\right)}}, & k^{\prime} \leqq y<1,
\end{array}
$$

$\operatorname{com} k^{\prime}=\sqrt{1-k^{2}}$.

O número $k$ que aparece em (1.6) é chamado de módulo elíptico. Este número pode ser tomado como sendo real ou imaginário puro. Mas, em aplicações físicas e engenheria, mediante algumas transformações, pode ser tomado no intervalo $(0,1)$. Ao longo do trabalho, consideraremos $k \in(0,1)$. O módulo elíptico complementar, denotado por $k^{\prime}$, é a quantidade $k^{\prime}=\sqrt{1-k^{2}}$. 


\section{Capítulo 2}

\section{Boa colocação em $H^{1}(\mathbb{R})$}

Provaremos neste capítulo a boa colocação global em $H^{1}(\mathbb{R})$ do (PVI) associado à equação (7). A base dos resultados é, como mencionado no capítulo anterior, os Teoremas 1.4 e 1.5.

Começaremos fazendo uma descrição rigorosa do operador

$$
-\Delta_{ \pm c, Z} \equiv H_{Z}=-\frac{d^{2}}{d x^{2}}-Z\left[\delta_{c}+\delta_{-c}\right] ; \quad(c, Z) \in(0, \infty) \times \mathbb{R} \backslash\{0\},
$$

definido em $L^{2}(\mathbb{R})$ e cujo domínio é $\mathcal{D}\left(H_{Z}\right)=\mathcal{D}$, sendo

$$
\mathcal{D}=\left\{f \in H^{1}(\mathbb{R}) \cap H^{2}(\mathbb{R} \backslash\{ \pm c\}): f^{\prime}\left( \pm c^{+}\right)-f^{\prime}\left( \pm c^{-}\right)=-Z f( \pm c)\right\}
$$

Em seguida, mostramos que $H_{Z}$ não possui autovalores negativos para o caso em que $Z<0$. Como aplicação do Teorema 1.5 , mostraremos que se $Z \in\left(0, \frac{1}{c}\right]$, então o operador $H_{Z}$ possui exatamente um autovalor negativo, e se $Z \in\left(\frac{1}{c}, \infty\right)$, então $H_{Z}$ possui exatamente dois autovalores negativos. Para a boa colocação local em $H^{1}(\mathbb{R})$, usaremos os fatos obtidos sobre o espectro de $H_{Z}$ para definirmos uma norma apropriada que seja equivalente à norma usual em $H^{1}(\mathbb{R})$. Por fim, as quantidades conservadas (carga e energia) que decorrem do Teorema 1.4 (ver (11) e (12)) garantiram a boa colocação global em $H^{1}(\mathbb{R})$.

\section{$2.1 \quad \mathrm{O}$ operador $H_{Z}$}

Considere o operador $\mathcal{H}=-\frac{d^{2}}{d x^{2}}$ sobre $L^{2}(\mathbb{R})$, com domínio $D(\mathcal{H})=H^{2}(\mathbb{R})$ e o operador restrição

$$
\left\{\begin{array}{l}
\left.\mathcal{H}_{0} \equiv \mathcal{H}\right|_{D\left(\mathcal{H}_{0}\right)}, \\
D\left(\mathcal{H}_{0}\right)=\{g \in D(\mathcal{H}): g(c)=g(-c)=0\} .
\end{array}\right.
$$

O adjunto de $\mathcal{H}_{0}$ vem dado por (ver [3, seção I.3.1])

$$
\left\{\begin{array}{l}
\mathcal{H}_{0}^{*}=-\frac{d^{2}}{d x^{2}}, \\
D\left(\mathcal{H}_{0}^{*}\right)=H^{1}(\mathbb{R}) \cap H^{2}(\mathbb{R} \backslash\{ \pm c\}) .
\end{array}\right.
$$

Seja $c>0$. Então, por definição, para $\psi \in H^{1}(\mathbb{R}) ;\left\langle\delta_{c}, \psi\right\rangle=\psi(c)$, e $\left|\left\langle\delta_{c}, \psi\right\rangle\right| \leq\|\psi\|_{H^{1}}$. Logo, as interações delta $\delta_{ \pm c}$ definem dois funcionais contínuos em $H^{1}(\mathbb{R})$. Portanto, $\delta_{ \pm c} \in H^{-1}(\mathbb{R})$. 
Definição 2.1 Seja $\mathcal{A}_{0}$ um operador simétrico, fechado e densamente definido sobre um espaço de Hilbert X. Denotemos por $A_{0}^{*}$ o seu adjunto. Consideremos os subespaços

$$
D_{+}=\operatorname{ker}\left(A_{0}^{*}-i\right), \quad \text { e } \quad D_{-}=\operatorname{ker}\left(A_{0}^{*}+i\right) .
$$

$D_{+}$e $D_{-}$são chamados os subespaços de deficiência associados ao operador $A_{0}$. Chamamos de indice de deficiência de $A_{0}$, ao par de números

$$
\left(n_{+}\left(A_{0}\right), n_{-}\left(A_{0}\right)\right),
$$

onde $n_{+}\left(A_{0}\right)=\operatorname{dim} \operatorname{ker}\left(D_{+}\right)$e $n_{-}\left(A_{0}\right)=\operatorname{dim} \operatorname{ker}\left(D_{-}\right)$.

O seguinte Lema pode ser provado fazendo uma adaptação da prova feita em Alveberio et al. ([3]), bem como em Angulo\&Ponce ([5]), para o caso da interação delta centrada na origem, i.e., quando $c=0$. Com as notações acima, temos o seguinte resultado.

Lema 2.2 Sejam $X=L^{2}(\mathbb{R}), A_{0} \equiv \mathcal{H}_{0}$ e $D\left(A_{0}\right)=\mathcal{D}\left(\mathcal{H}_{0}\right)$. Então, $A_{0}$ tem as seguintes propriedades:

1. $\overline{\Gamma\left(A_{0}\right)}=\Gamma\left(A_{0}\right)$, sendo $\Gamma\left(A_{0}\right)$ o gráfico de $A_{0}$. Logo, o operador $A_{0}$ é fechado.

2. $\left\langle A_{0} g, h\right\rangle=\left\langle g, A_{0} h\right\rangle$, para todo $g, h \in D\left(A_{0}\right)$. (Simetria)

3. $\overline{D\left(A_{0}\right)}=L^{2}(\mathbb{R})$. (Densidade).

4. $n_{ \pm}\left(A_{0}\right)=2$

O operador $H_{Z}$ pode ser entendido então como uma extensão auto adjunta do operador restrição definido em (2.3) da seguinte forma, para $\psi \in \mathcal{D}\left(H_{Z}\right)$;

$$
\left\{\begin{array}{l}
H_{Z} \psi=-\frac{d^{2} \psi}{d x^{2}}(x), \quad x \in \mathbb{R} \backslash\{ \pm c\} \\
\psi^{\prime}\left( \pm c^{+}\right)-\psi^{\prime}\left( \pm c^{-}\right)=-Z \psi( \pm c) .
\end{array}\right.
$$

Vamos agora usar o Teorema 1.5 dado em Alveberio ([3]) para obter que o espectro pontual, $\sigma_{p}\left(H_{Z}\right)$ do operador $H_{Z}$, satisfaz $\sigma_{p}\left(H_{Z}\right) \subset(-\infty, 0)$. De fato, para $E_{0} \in \sigma_{p}\left(H_{Z}\right)$, com $E_{0}=k_{0}^{2}<0$, temos para $f \in \operatorname{ker}\left(A_{0}^{*}-k_{0}^{2} I\right)=\operatorname{ker}\left(\mathcal{H}_{0}^{*}-k_{0}^{2} I\right)$, que $-f^{\prime \prime}(x)=k_{0}^{2} f(x)$. Logo, do Teorema 1.5 em Albeverio [3] temos que as soluções desta equação estão geradas pelas funções

$$
\left\{\frac{i}{2 k_{0}} e^{i k_{0}|x-c|}, \frac{i}{2 k_{0}} e^{i k_{0}|x+c|}\right\} \operatorname{com} \operatorname{Im}\left(k_{0}\right)>0 \text {. }
$$

Portanto,

$$
f(x)=f_{k_{0}}(x)=c_{1} \frac{i}{2 k_{0}} e^{i k_{0}|x-c|}+c_{2} \frac{i}{2 k_{0}} e^{i k_{0}|x+c|},
$$

onde o par $\left(c_{1}, c_{2}\right)^{T}$ é autovetor da matriz

$$
\Gamma_{\gamma, X}\left(k_{0}\right)=\left(\begin{array}{cc}
\frac{1}{Z}-\frac{i}{2 k_{0}} & -\frac{i}{2 k_{0}} e^{2 i k_{0} c} \\
-\frac{i}{2 k_{0}} e^{2 i k_{0} c} & \frac{1}{Z}-\frac{i}{2 k_{0}}
\end{array}\right),
$$


sendo $\gamma=\{-Z, Z\}$ e $X=\{-c, c\}$, e que o determinante de $\Gamma_{\gamma, X}\left(k_{0}\right)$ deve ser zero, i.e.,

$$
\begin{aligned}
\operatorname{det}\left(\Gamma_{\gamma, X}\left(k_{0}\right)\right) & =\left(\frac{1}{Z}-\frac{i}{2 k_{0}}\right)^{2}+\frac{1}{4 k_{0}^{2}} e^{4 i k_{0} c} \\
& =\frac{1}{Z^{2}}-\frac{1}{\sqrt{\left|E_{0}\right|} Z}-\frac{1}{4 E_{0}}+\frac{1}{4 E_{0}} e^{-4 c \sqrt{\left|E_{0}\right|}}=0 .
\end{aligned}
$$

Se $n\left(H_{Z}\right)$ representa o número de autovalores negativos do operador $H_{Z}$, pode-se mostrar que $n\left(H_{Z}\right)=n(D)$; sendo $D$ a matriz

$$
\left(\begin{array}{cc}
-Z+\frac{1}{2 c} & -\frac{1}{2 c} \\
-\frac{1}{2 c} & -Z+\frac{1}{2 Z}
\end{array}\right)
$$

onde $n(D)$ é o número de autovalores negativos da matriz $D$. Um cálculo mostra que

$$
\begin{cases}n(D)=0, & \text { se } Z<0 \\ n(D)=1, & \text { se } Z \in\left(0, \frac{1}{c}\right] \\ n(D)=2, & \text { se } Z \in\left(\frac{1}{c}, \infty\right)\end{cases}
$$

O fato do operador $H_{Z}$ não possuir autovalores negativos, no caso em que $Z<0$, segue-se como consequência da sua forma quadrática, a saber: para $f \in \mathcal{D}\left(H_{Z}\right)$, temos

$$
\left\langle H_{Z} f, f\right\rangle_{2}=-\int_{\mathbb{R}} f^{\prime \prime}(x) f(x) d x=-Z\left[|f(-c)|^{2}+|f(c)|^{2}\right]+\int_{\mathbb{R}}\left(f^{\prime \prime}\right)^{2} d x .
$$

Já que para $Z<0,\left\langle H_{Z} f, f\right\rangle_{2} \geqq 0$, tem-se que o operador $H_{Z}$ não possui autovalores negativos.

Devido à existência dos perfis de tipo Jacobiano no intervalo $[-c, c]$ (ver Capítulo 3 ), consideraremos apenas o caso em que $Z \in\left(0, \frac{\pi}{4 c}\right) \subset\left(0, \frac{1}{c}\right]$. Portanto, $n\left(H_{Z}\right)=1$.

\subsection{Teoria de boa colocação global}

A seguir estabeleceremos nosso resultado de boa colocação global para o problema

$$
\left\{\begin{array}{l}
i \partial_{t} u=H_{Z} u-|u|^{2} u \\
u(0, x)=u_{0}(x)
\end{array}\right.
$$

em $H^{1}(\mathbb{R})$, sendo $H_{Z}$ o operador definido em (3.1), o qual é entendido como indica (2.4). De fato, a partir do Teorema 1.4 e aproveitando as leis de conservação da carga $\mathcal{N}$, definida em (12), e da energia $\mathcal{H}$, definida em (11), é possível mostrar que o problema (2.5) é globalmente bem posto em $H^{1}(\mathbb{R})$. Notamos que pode também ser mostrado um resultado de boa colocação local em $H^{1}(\mathbb{R})$ usando ferramentas do espalhamento inverso baseados no recente trabalho de Duchene\&Marzuola\&Weinstein ([22]).

Teorema 2.3 Seja $Z \in\left(-\infty, \frac{\pi}{4 c}\right)$. Para qualquer $u_{0} \in H^{1}(\mathbb{R})$, existe $T=T\left(\left\|u_{0}\right\|_{1}\right)>0$ e uma única solução $u \in C\left([0, T) ; H^{1}(\mathbb{R})\right)$ de $(2.5)$ tal que $u(0)=u_{0}$. Além disso, para todo $T^{\prime}<T$, existe $\delta>0$ tal que a aplicação dado-solução

$$
\tilde{u}_{0} \in B\left(u_{0} ; \delta\right) \mapsto \tilde{u} \in C\left(\left[0, T^{\prime}\right) ; H^{1}(\mathbb{R})\right)
$$


é contínua. Mais ainda, as quantidades

$$
\mathcal{H}(u)=\frac{1}{2}|| u_{x} \|_{2}^{2}-\frac{Z}{2}\left[|u(c)|^{2}+|u(-c)|^{2}\right]-\frac{1}{4}|u|_{L^{4}}^{4} \quad(\text { Energia })
$$

$e$

$$
\mathcal{N}(u)=\frac{1}{2} \int_{\mathbb{R}}|u(x)|^{2} d x \quad(\text { Carga })
$$

são conservadas, i.e., $\mathcal{H}(u(t))=\mathcal{H}\left(u_{0}\right)$ e $\mathcal{N}(u(t))=\mathcal{N}\left(u_{0}\right)$, para todo $t \in[0, T)$.

Finalmente, se o dado inicial $u_{0}$ é par, então a solução u(t) também é par.

Demonstração. Sejam $Z \in\left(-\infty, \frac{\pi}{4 c}\right)$ e $\gamma<0$ o único autovalor negativo de $H_{Z}$, quando $0<Z<\frac{\pi}{4 c}$. Então $H_{Z} \geq \eta$, onde $\eta=0$ se $Z \leqq 0$ e $\eta=\gamma$ se $Z>0$. Desta forma, para o operador autoadjunto $A \equiv-H_{Z}+\eta$ sobre $L^{2}(\mathbb{R})$, com domínio $D(A)=\mathcal{D}$ definido em $(2.2)$, temos $A \leq 0$. Seja $X_{A} \equiv H^{1}(\mathbb{R})$ dotado com a norma

$$
\begin{aligned}
\|u\|_{X_{A}}^{2} & =\|u\|_{2}^{2}-\langle A u, u\rangle_{2}=\|u\|_{2}^{2}+\left\langle H_{Z} u, u\right\rangle-\eta\|u\|_{2}^{2} \\
& =\left\|u_{x}\right\|_{2}^{2}+(1-\eta)\|u\|_{2}^{2}-Z\left[|u(c)|^{2}+|u(-c)|^{2}\right] .
\end{aligned}
$$

De fato, pelo princípio do mini-max temos, para $\frac{\pi}{4 c}>Z>0$

$$
\gamma=\inf \left\{\left\|v_{x}\right\|^{2}-Z\left[\left[|u(c)|^{2}+|u(-c)|^{2}\right]:\|v\|=1, v \in H^{1}(\mathbb{R})\right\} .\right.
$$

Portanto, para cada $Z \in\left(-\infty, \frac{\pi}{4 c}\right)$, existem números $C_{i}=C_{i}(Z)>0, i=1,2$, tais que

$$
C_{1}\|u\|_{H^{1}}^{2} \leq\|u\|_{X_{A}}^{2} \leq C_{2}\|u\|_{H^{1}}^{2}
$$

Então, a norma $\|\cdot\|_{X_{A}}$ definida em $X_{A}$, é equivalente à norma usual em $H^{1}(\mathbb{R})$.

Agora, se definimos $g: X_{A} \longrightarrow L^{2}(\mathbb{R})$ por $g(u)=|u|^{2} u$, então $g \in C\left(X_{A}, L^{2}(\mathbb{R})\right)$ e

$$
\|g(v)-g(u)\|_{2} \leq C\left(\|v\|_{X_{A}}^{2}+\|u\|_{X_{A}}^{2}\right)\|u-v\|_{2},
$$

para quaisquer $u, v \in X_{A}$. Além disso, $g=G^{\prime}$, onde $G \in C^{1}\left(X_{A}, \mathbb{R}\right)$ está definida por $G(u)=\frac{1}{4}|u|^{4}$. Finalmente, para qualquer $u \in X_{A}, g(u) \bar{u}=|u|^{4}$, então

$$
\langle g(u), i u\rangle_{2}=-i \int_{\mathbb{R}} g(u) \bar{u} .
$$

Segue-se que $\operatorname{Re}\left(\langle g(u), i u\rangle_{2}\right)=0$. Portanto, a boa colocação local para o problema (2.5), em $H^{1}(\mathbb{R})$, segue-se do Teorema 1.4, Teorema 3.7.1 em Cazenave [14] e das estimativas de tipo Strichartz em Kovař́k\&\&Sacchetti [33].

A boca coloção global para o problema $(2.5)$, em $H^{1}(\mathbb{R})$, segue-se de argumentos standard baseados na desigualdade de Gagliardo-Nirenberg, o Teorema de imersão de Sobolev e das leis de conservação da carga (12) e da energia (11). 


\section{Capítulo 3}

\section{Existência de soluções de tipo peak-standing waves}

Neste capítulo vamos mostrar a existência de uma solução do tipo standing-wave, $u(x, t)=$ $e^{i \omega t} \Phi_{\omega, Z, c}(x)$, para a equação NLS- $\delta_{ \pm c}$ dada em (7), de tal maneira que as condições em (8) sejam satisfeitas. Essas soluções irão a pertencer ao domínio do operador $-\frac{d^{2}}{d x^{2}}-Z\left[\delta_{c}+\delta_{-c}\right]$, $Z \neq 0$

$$
-\Delta_{ \pm c, Z} \equiv H_{Z}=-\frac{d^{2}}{d x^{2}}-Z\left[\delta_{c}+\delta_{-c}\right] ; \quad(c, Z) \in(0, \infty) \times \mathbb{R} \backslash\{0\} .
$$

Nossa análise baseia-se na teoria das funções elípticas de Jacobi. Desta maneira, procuramos um perfil $\Phi_{\omega, Z, c}: \mathbb{R} \longrightarrow \mathbb{R}$ que satisfaça o seguinte problema

$$
\left\{\begin{array}{l}
\Phi_{\omega, Z, c}^{\prime \prime}(x)-\omega \Phi_{\omega, Z, c}(x)+\Phi_{\omega, Z, c}^{3}(x)=0, \quad \forall x \in \mathbb{R}-\{ \pm c\} \\
\Phi_{\omega, Z, c}^{\prime}\left( \pm c^{+}\right)-\Phi_{\omega, Z, c}^{\prime}\left( \pm c^{-}\right)=-Z \Phi_{\omega, Z, c}( \pm c)
\end{array}\right.
$$

e $\lim _{|x| \rightarrow \infty} \Phi_{\omega, Z, c}(x)=0$.

O caso $Z \neq 0$ e $c=0$ foi estudado por Fukuizumi, Jeanjean, Le Coz, Ohta (ver [19], [20], [35]) para a equação NLS- $\delta(5)(\operatorname{com} p=2)$. De fato, foi mostrado que a única solução de (3.2) $\operatorname{com} c=0$, módulo rotações, está dada pela função

$$
\Phi_{\omega, Z, 0}(x)=\sqrt{2 \omega} \operatorname{sech}\left(\sqrt{\omega}|x|+\tanh ^{-1}\left(\frac{Z}{2 \sqrt{\omega}}\right)\right),
$$

para $\omega>Z^{2} / 4$. Para $Z=0$, o conjunto de soluções suaves para (3.2) sobre $\mathbb{R}$ possui diversos trabalhos relevantes na literatura. De fato, existe uma única solução positiva, $\Psi_{\omega}$, módulo translações e rotações, de (3.2), dada pelo soliton

$$
\Psi_{\omega}(x)=\sqrt{2 \omega} \operatorname{sech}(\sqrt{\omega} x), \quad \omega>0,
$$

associada à equação cúbica de Schrödinger

$$
i \partial_{t} u+u_{x x}=-|u|^{2} u .
$$

É conhecido que os perfis peak-standing waves $e^{i \omega t} \Phi_{\omega, Z, 0}$ em (3.3), são orbitalmente estáveis para $Z>0$ e orbitalmente instáveis para $Z<0$ (ver [19], [20], [35]). Mais ainda, Cazenave e Lions em [13] mostraram que para $Z=0$, o soliton $e^{i \omega t} \Psi_{\omega}$, é orbitalmente estável para todo $\omega>0$.

Mostraremos a seguir como é possível construir uma família de soluções para o problema (3.2). Em primeiro lugar, consideremos $x \in(-\infty,-c) \cup(c, \infty)$. Baseados na solução dada em (3.4), determinamos uma certa translação desta função. Já para $x \in[-c, c]$, consideramos um perfil elíptico de tipo dnoidal ou do tipo cnoidal . 


\subsection{Existência de um perfil periódico entre os pontos de interação}

Nesta seção mostraremos a existência de um perfil par para o problema de fronteira (3.2), com $Z \neq 0$. Segue imediatamente de (3.4) que o perfil

$$
\Phi_{\omega, Z, c}(x)=\left\{\begin{array}{lrr}
\Psi_{\omega,-b}(x)=\sqrt{2 \omega} \operatorname{sech}(\sqrt{\omega}(x+c-b)), & \text { se } & -\infty<x<-c \\
\Psi_{\omega, b}(x)=\sqrt{2 \omega} \operatorname{sech}(\sqrt{\omega}(x-c+b)), & \text { se } & c<x<+\infty .
\end{array}\right.
$$

satisfaz a equação (3.2) para todo $x \in(-\infty,-c) \cup(c, \infty)$. Consideramos uma translação de (3.4) dada pelo parâmetro $b$. Segue imediatamente desta consideração que

$$
\lim _{|x| \rightarrow \infty} \Phi_{\omega, Z, c}(x)=\lim _{|x| \rightarrow \infty} \Phi_{\omega, Z, c}^{\prime}(x)=0 .
$$

Enfrentamos então o problema de establecer uma função, $\phi$, definida no intervalo $[-c, c]$ que satisfaça as seguintes propriedades:

1. $\phi(c)=\Psi_{\omega, b}\left(c^{+}\right), \quad \phi(-c)=\Psi_{\omega,-b}\left(-c^{-}\right)$;

2. $\Psi_{\omega, b}^{\prime}\left(c^{+}\right)-\phi^{\prime}\left(c^{-}\right)=-Z \phi(c), \phi^{\prime}\left(-c^{+}\right)-\Psi_{\omega,-b}^{\prime}\left(-c^{-}\right)=-Z \phi(-c)$.

Dividiremos nosso trabalho em dois casos: o caso $Z>0$ e o caso $Z<0$.

\subsubsection{Perfil dnoidal para $Z>0$}

Consideremos, para $x \in[-c, c]$, a função

$$
\Phi_{\omega, Z, c}(x)=\phi_{\alpha, \beta, \theta}(x)=\alpha d n(\beta x+\theta ; k),
$$

e suponhamos que $\Phi_{\omega, Z, c}$ é solução de (3.2). É importante recordar que o perfil dnoidal é positivo na reta toda. Os parâmetros $\alpha, \beta, \theta$ and $b$ que aparecem em (3.5) e em (3.6), serão determinados tanto da hipótese de que o nosso perfil é par, bem como das propriedades da função dnoidal e das condições de fronteira. Note que para $x \in(-c, c)$ temos

$$
\left\{\begin{array}{c}
\phi_{\alpha, \beta, \theta}^{\prime}(x)=-\alpha \beta k^{2} c n(\beta x+\theta ; k) \operatorname{sn}(\beta x+\theta ; k) \\
\phi_{\alpha, \beta, \theta}^{\prime \prime}(x)=\alpha \beta k^{2} d n(\beta x+\theta ; k)\left(1-2 c n^{2}(\beta x+\theta ; k)\right),
\end{array}\right.
$$

onde $\gamma \mapsto c n(\gamma ; k)$ e $\gamma \mapsto s n(\gamma ; k)$, para $\gamma \in \mathbb{R}$, são as funções elípticas de Jacobi cnoidal e snoidal, respectivamente. Segue que, se $\phi_{\alpha, \beta, \theta}$ satisfaz $(3.2)$ em $(-c, c)$, então

$$
\alpha^{3} d n(\beta x+\theta ; k)\left[d n^{2}(\beta x+\theta ; k)-\frac{\omega}{\alpha^{2}}+\frac{\beta^{2} k^{2}}{\alpha^{2}}\left(1-2 c n^{2}(\beta x+\theta ; k)\right)\right]=0 .
$$

É claro que com o objetivo de que $\phi_{\alpha, \beta, \theta}$ não seja a função nula (nem uma função constante) podemos supor que $\alpha \beta \neq 0$. Logo, da expressão anterior concluímos que

$$
d n^{2}(\beta x+\theta ; k)-\frac{2 \beta^{2} k^{2}}{\alpha^{2}} c n^{2}(\beta x+\theta ; k)=\frac{\omega-\beta^{2} k^{2}}{\alpha^{2}} .
$$

Vamos usar aqui uma identidade muito útil das funções elípticas de Jacobi, a saber: para $\gamma \in \mathbb{R}$ vale $d n^{2}(\gamma ; k)-k^{2} c n^{2}(\gamma ; k)=1-k^{2}$. Notamos da última expressão que, para 
tornar ela uma identidade em $(-c, c)$, isto é, para que tal expressão seja verificada para todo $x \in(-c, c)$; necessário é que

$$
k^{2}=\frac{2 \beta^{2} k^{2}}{\alpha^{2}}
$$

$\mathrm{e}$

$$
1-k^{2}=\frac{\omega-\beta^{2} k^{2}}{\alpha^{2}}
$$

Portanto,

$$
\alpha^{2}=\frac{2 \omega}{2-k^{2}} \text { e } \beta^{2}=\frac{\omega}{2-k^{2}} .
$$

Segue que $\phi_{\alpha, \beta, \theta}$ verifica a equação elíptica (3.2) para todo $x \in(-c, c)$. Pela expressão explícita de $\Phi_{\omega, Z, c}$ em (3.5), e da continuidade de $\Phi_{\omega, Z, c}$ nos pontos $x= \pm c$, escolhemos $\alpha>0$. $\log \alpha=\sqrt{\frac{2 \omega}{2-k^{2}}}$. Agora, com o objetivo de que o nosso perfil dnoidal, $\Phi_{\omega, Z, c}$, seja uma função par, e já que o período minimal real da função dnoidal é $2 K(k)$, então as únicas escolhas possíves do parâmetro $\theta$ são: $\theta=0$ ou $\theta=K(k)$. A Figura 3.1 mostra o exemplo da função $\phi_{\alpha, \beta, \theta}$ para os casos $\theta=0$ e $\theta=K(k)$. Devido à paridade do perfil $\Phi_{\omega, Z, c}$

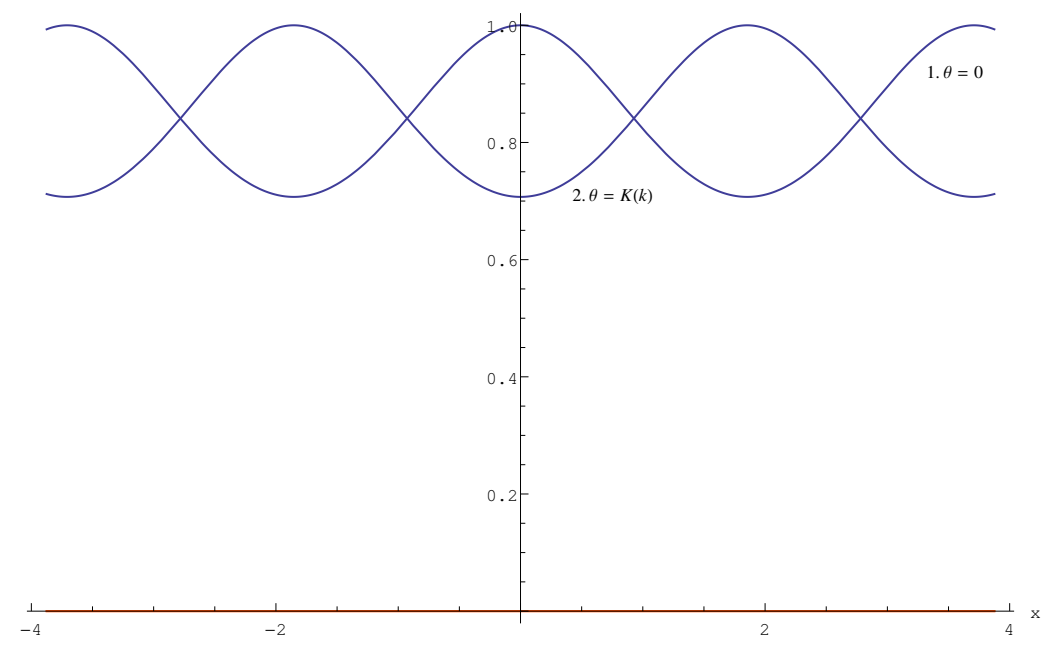

Figura 3.1: $\phi_{\alpha, \beta, \theta} \operatorname{com} \theta=0$ e $\theta=K(k)$. Aqui, $\alpha=1$ e $\beta=\frac{1}{\sqrt{2}}$

podemos escolher então $\beta>0$. Desta forma: se $\phi_{\alpha, \beta, \theta}$ é um perfil dnoidal, par, e que satisfaz a equação elíptica dada em (3.2) então

$$
\alpha=\sqrt{\frac{2 \omega}{2-k^{2}}}, \beta=\sqrt{\frac{\omega}{2-k^{2}}}, \text { e } \theta=0 \text { ou } \theta=K(k) .
$$

Consideremos o caso particular em que $\theta=0$. Então o nosso perfil de tipo peak-standing wave satisfaz

$$
\Phi_{\omega, Z, c}(x)=\left\{\begin{array}{lcc}
\Psi_{\omega,-b}(x)=\sqrt{2 \omega} \operatorname{sech}(\sqrt{\omega}(x+c-b)), & \text { se } & -\infty<x<-c \\
\phi_{\alpha, \beta, \theta}(x)=\phi_{\alpha, \beta}(x)=\alpha d n(\beta x ; k), & \text { se } \quad-c \leq x \leq c \\
\Psi_{\omega, b}(x)=\sqrt{2 \omega} \operatorname{sech}(\sqrt{\omega}(x-c+b)), & \text { se } & c<x<+\infty,
\end{array}\right.
$$

sendo $\alpha$ e $\beta$ dados em (3.7). É importante notar que, para $\omega>0$ fixado, a maneira como estabelecemos o perfil dnoidal $\Phi_{\omega, Z, c}$, permite obter uma família não enumerável de funções 
$\phi_{\alpha, \beta, \theta}$ (determinadas pelo número $k \in(0,1)$ ) que satisfazem a equação elíptica (3.2) no intervalo $(-c, c)$. Além do mais, dado que

$$
\lim _{k \rightarrow 1^{-}} d n(\gamma ; k)=\operatorname{sech}(\gamma)
$$

para todo $\gamma \in \mathbb{R}$, então o perfil secante hiperbólica é a solução limite dentro desta família.

Vamos agora tratar as condições de continuidade e de fronteira que deve satisfazer o perfil $\Phi_{\omega, Z, c}$. Isto vai mostrar, como é de se esperar, certas relações entre os parâmetros $\alpha, \beta, \theta, b$ e $Z$ que aparecem envolvidos em nosso modelo. Começamos com a condição de continuidade. É claro que para $x \in \mathbb{R} \backslash\{ \pm c\}$, o perfil $\Phi_{\omega, Z, c}(x)$ dado em (3.8) é contínuo. Nos únicos pontos onde, a priori, $\Phi_{\omega, Z, c}$ não é contínuo são de fato $x= \pm c$. Denotaremos as funções elípticas de Jacobi sem explicitar o seu módulo; de tal maneira que $\operatorname{sn}(\gamma)$, $c n(\gamma)$ e $d n(\gamma)$ representarão as respectivas funções $\operatorname{sn}(\gamma ; k), c n(\gamma ; k)$ e $d n(\gamma ; k)$; deixando a notação usual (usando o módulo) só naquelas circunstâncias em que seja importante ressaltá-lo. Requeremos a seguinte

1) Condição de continuidade: necessário é que $\Phi_{\omega, Z, c}\left( \pm c^{+}\right)=\Phi_{\omega, Z, c}\left( \pm c^{-}\right)$. Isto equivale à expressão

$$
\operatorname{sech}(\sqrt{\omega} b)=\frac{d n(\beta c)}{\sqrt{2-k^{2}}}
$$

A seguinte condição garante que $\Phi_{\omega, Z, c}^{\prime}$ satisfaz a condição de salto.

2) Condição de salto na derivada: $\Phi_{\omega, Z, c}^{\prime}\left( \pm c^{+}\right)-\Phi_{\omega, Z, c}^{\prime}\left( \pm c^{-}\right)=-Z \Phi_{\omega, Z, c}( \pm c)$. Isto acarreta a equação

$$
-\sqrt{\omega} \operatorname{sech}(\sqrt{\omega} b) \tanh (\sqrt{\omega} b)+\frac{\sqrt{\omega} k^{2}}{2-k^{2}} \operatorname{cn}(\beta c) \operatorname{sn}(\beta c)=-Z \operatorname{sech}(\sqrt{\omega} b)
$$

ou ainda,

$$
\frac{\omega k^{4} c n^{2}(\beta c) \operatorname{sn}^{2}(\beta c)}{\left(2-k^{2}\right)^{2}}=(\sqrt{\omega} \tanh (\sqrt{\omega} b)-Z)^{2} \operatorname{sech}^{2}(\sqrt{\omega} b) .
$$

Dado que $d n^{2}(\gamma)-k^{2} c n^{2}(\gamma)=1-k^{2}$ e $d n^{2}(\gamma)=1-k^{2} s n^{2}(\gamma)(\gamma \in \mathbb{R})$, a equação anterior fica

$$
\frac{\omega\left(d n^{2}(\beta c)+k^{2}-1\right)\left(1-d n^{2}(\beta c)\right)}{\left(2-k^{2}\right)^{2}}=(\sqrt{\omega} \tanh (\sqrt{\omega} b)-Z)^{2} \operatorname{sech}^{2}(\sqrt{\omega} b) .
$$

Usando a condição de continuidade (3.9), obtemos

$$
\begin{array}{r}
\frac{\omega\left[\left(2-k^{2}\right) \operatorname{sech}^{2}(\sqrt{\omega} b)+\right.}{\left.k^{2}-1\right]\left[1-\left(2-k^{2}\right) \operatorname{sech}^{2}(\sqrt{\omega} b)\right]} \\
\left(2-k^{2}\right)^{2} \\
(\sqrt{\omega} \tanh (\sqrt{\omega} b)-Z)^{2} \operatorname{sech}^{2}(\sqrt{\omega} b) .
\end{array}
$$

Decorre desta equação que

$$
2 Z \sqrt{\omega}\left(\left(2-k^{2}\right) \operatorname{sech}(\sqrt{\omega} b)\right)^{2} \tanh (\sqrt{\omega} b)=\omega\left(1-k^{2}\right)+Z^{2}\left(2-k^{2}\right)^{2} \operatorname{sech}^{2}(\sqrt{\omega} b)>0 .
$$

Finalmente, segue desta última expressão que

$$
Z \tanh (\sqrt{\omega} b)>0, \quad \text { ou equivalentemente, } \quad b Z>0 .
$$




\subsection{EXISTÊNCIA DE UM PERFIL PERIÓDICO ENTRE OS PONTOS DE INTERAÇÃO17}

Isto mostra un fato interessante: o sinal de $Z$ determina a forma da curva $\Phi_{\omega, Z, c}$ entorno dos pontos $x= \pm c$. Por exemplo, suponhamos que $\omega=2$ e $c=1$. Então $f(x)=2 \operatorname{sech}(\sqrt{2}(x-1))$ é uma translação à direita da função $x \mapsto 2 \operatorname{sech}(\sqrt{2} x)$. Suponhamos que $Z>0$, então (3.14) implica que $b>0$, assim, fixemos o valor $b=0,5$. Desta maneira, $h(x)=2 \operatorname{sech}(\sqrt{2}(x-0,5))$ é uma translação à direita de $f$. A Figura 3.2 mostra os gráficos de $f$ e $h$. Apartir deste exemplo podemos concluir que, de maneira geral, para $Z>0$, o ponto maximal da função $\Psi_{\omega, b}$ em (3.8), quando considerada na reta, está à esquerda do ponto $x=c$. Logo então, $\Psi_{\omega, b}^{\prime}\left(c^{+}\right)<0$. Agora, ainda neste caso, o ponto maximal de $\Psi_{\omega,-b}$ em (3.8) está à direita do ponto $x=-c$; então $\Psi_{\omega,-b}^{\prime}\left(-c^{-}\right)>0$.

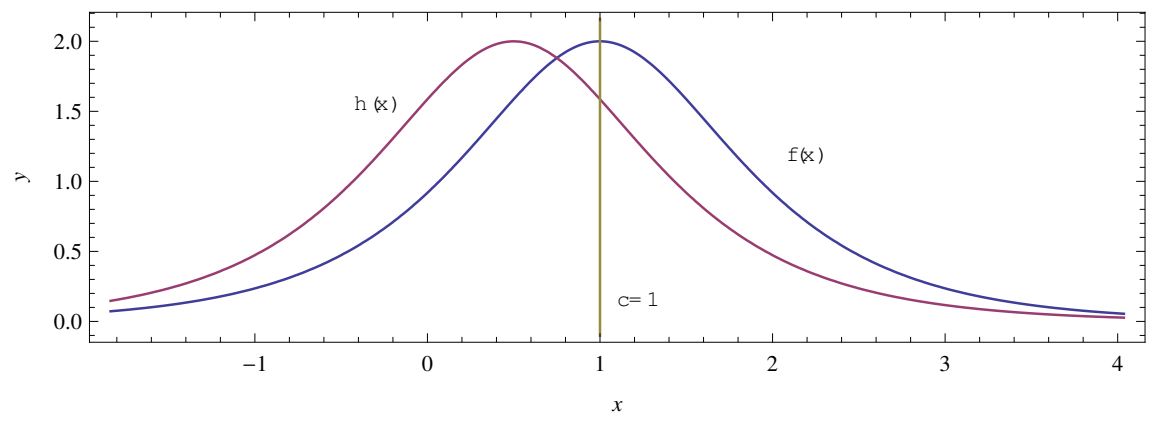

Figura 3.2: Gráficos de $f$ e $h$

Por outro lado, $Z<0 \leftrightarrow b<0$, então o ponto maximal da função $\Psi_{\omega, b}$ em (3.8) (quando considerada na reta toda) está à direita do ponto $x=c$. Assim, $\Psi_{\omega, b}^{\prime}\left(c^{+}\right)>0$. Finalmente, neste caso, o ponto maximal de $\Psi_{\omega,-b}$ está à esquerda do ponto $x=-c, \operatorname{logo} \Psi_{\omega,-b}^{\prime}\left(-c^{-}\right)<0$. A conclusão disto é:

se $\mathrm{Z}>0$, então

$$
\left\{\begin{array}{l}
\Psi_{\omega, b}^{\prime}\left(c^{+}\right)<0 ; \\
\Psi_{\omega,-b}^{\prime}\left(-c^{-}\right)>0 .
\end{array}\right.
$$

Se $Z<0$, então

$$
\left\{\begin{array}{l}
\Psi_{\omega, b}^{\prime}\left(c^{+}\right)>0 ; \\
\Psi_{\omega,-b}^{\prime}\left(-c^{-}\right)<0 .
\end{array}\right.
$$

De fato, de (3.8) deduzimos que

$$
\Psi_{\omega, b}^{\prime}\left(c^{+}\right)=-\Psi_{\omega,-b}^{\prime}\left(-c^{-}\right) .
$$

Mais ainda, pela condição de fronteira (salto na derivada) dada em (3.10), é possível deduzir que

$$
\phi_{\alpha, \beta}^{\prime}\left(c^{+}\right)=-\phi_{\alpha, \beta}\left(-c^{-}\right) .
$$

Até aqui vemos a maneira em que podem ser determinados os parâmetros $\alpha, \beta, \theta$ e $b$ para o modelo dnoidal proposto em (3.8), a partir das identidades das funções elípticas de Jacobi e das condições de continuidade e de salto na derivada dadas em (3.9) e (3.10), respectivamente.

Por outro lado, ainda nada foi dito sobre os parâmetros $c$ e $Z$ que aparecem no modelo dado em (3.2), exeto nos casos em que $(c, Z) \in(0, \infty) \times \mathbb{R} \backslash\{0\}$. É importante ressaltar que, com o objetivo de estabelecer nossos resultados de estabilidade/instabilidade não linear para 
as soluções standing-wave, iremos a usar a teoria de perturbação analítica, a qual permitirá fazer uma análise espectral do operador $\mathcal{L}_{1, Z, c}$ para $Z \rightarrow 0$. Por esta razão, será essencial que o perfil dnoidal, (3.8), convirja para o soliton da forma (3.4). A razão para este fato será então esclarecida: Seja $c>0$ fixado em (7). Esta condição de fixar o ponto de interação da delta, permitirá obter uma família de equações associadas a (7), a qual estará indexada pelo parâmetro $Z$. Para determinar o índice de Morse associado ao operador auto adjunto $\mathcal{L}_{1, Z, c}$ precisamos que o perfil dnoidal $\Phi_{\omega, c, Z}$ em (3.8) convirja para uma função da forma

$$
\Psi(x)=\sqrt{2 \omega^{*}} \operatorname{sech}\left(\sqrt{\omega^{*}} x\right), \text { quando } Z \rightarrow 0,
$$

para um número $\omega^{*}>0$ específico, pois para este tipo de funções (semelhante à solução associada à equação $(7)$, no caso em que $Z=0$ ) o espectro do operador correspondente associado,

$$
\mathcal{L}_{1, Z, 0}=-\frac{d^{2}}{d x^{2}}+\omega-3 \Psi^{2}
$$

é muito bem conhecido pelo Teorema da Oscilação de Sturm-Liouville. Portanto, assumiremos a existência de um certo número $\omega^{*}>0$, que será determinado explicitamente como função do parâmetro $c$, com a propriedade de que

$$
\lim _{Z \rightarrow 0} \Phi_{\omega, Z, c}(x)=\Psi(x), \quad \text { para } x \in \mathbb{R} \backslash\{ \pm c\} .
$$

Para este propósito é necessário estabelecer a seguinte proposição, que dá uma relação conveniente entre o ponto $c>0$ e o período minimal da onda dnoidal $\phi=\phi_{\alpha, \beta}$ dada em (3.8).

Proposição 3.1 Suponha que existe um número $\omega^{*}>0$ que satisfaz (3.17). Se $T_{\phi}$ é o período minimal da função dnoidal $\phi$, então $0<c \leq \frac{T_{\phi}}{2}$.

Demonstração. Como o período minimal real da função dnoidal $x \mapsto d n(x ; k)$ é $2 K(k)$, então o período minimal da onda $\phi$ será

$$
T_{\phi}=\frac{2 K(k)}{\beta}=2 K(k) \sqrt{\frac{2-k^{2}}{\omega}} .
$$

Suponha que $\frac{T_{\phi}}{2}<c$, então existem dois pontos $x \in\left[0, \frac{T_{\phi}}{2}\right)$ e $y \in\left(\frac{T_{\phi}}{2}, c\right)$ tais que $\phi(x)=$ $\phi(y)$. Pela hipótese, temos que para todo $\gamma \in[0, c), \phi(\gamma) \longrightarrow \sqrt{2 \omega^{*}} \operatorname{sech}\left(\sqrt{\omega^{*}} \gamma\right)$, quando $Z \rightarrow 0$. Em particular, para $x, y \in[0, c)$ devemos ter $\phi(x) \longrightarrow \sqrt{2 \omega^{*}} \operatorname{sech}\left(\sqrt{\omega^{*}} x\right)$ e $\phi(y) \longrightarrow$ $\sqrt{2 \omega^{*}} \operatorname{sech}\left(\sqrt{\omega^{*}} y\right)$. Portanto, $\operatorname{sech}\left(\sqrt{\omega^{*}} x\right)=\operatorname{sech}\left(\sqrt{\omega^{*}} y\right)$, o que é impossível dado que a função secante hiperbólica é estritamente descrescente no intervalo $[0,+\infty)$.

Observação 3.2 (Um perfil não conveniente) $S e c=\frac{T_{\phi}}{2}=\sqrt{\frac{2-k^{2}}{\omega}} K(k)$, então

$$
d n(\beta c ; k)=\sqrt{1-k^{2}}
$$

isto é, a função $\phi$ atinge o seu mínimo no ponto $x=c$, donde segue que $\phi^{\prime}\left(c^{-}\right)=0$. Portanto, a condição de continuidade (3.9) e a de salto na derivada (3.10) implicam

$$
\frac{\sqrt{1-k^{2}}}{\sqrt{2-k^{2}}}=\operatorname{sech}(\sqrt{\omega} b), \quad e \frac{Z}{\sqrt{\omega}}=\tanh (\sqrt{\omega} b),
$$


respectivamente. Segue que

$$
\frac{1-k^{2}}{2-k^{2}}+\frac{Z^{2}}{\omega}=1
$$

Por (3.18) deduzimos que $\alpha=\sqrt{\frac{2 \omega}{2-k^{2}}}=\sqrt{2}|Z|$ e $\beta=\sqrt{\frac{\omega}{2-k^{2}}}=|Z|$. Neste caso, o perfil $\Phi_{\omega, c, Z}$ em (3.8) fica dado por

$$
\Phi_{\omega, Z, c}(x)=\left\{\begin{array}{l}
|Z| \sqrt{2\left(2-k^{2}\right)} \operatorname{sech}\left(Z \sqrt{2-k^{2}}(x+c-b)\right), \quad \text { se } \quad-\infty<x<-c \\
\sqrt{2}|Z| d n\left(Z x ; k^{2}\right), \quad \text { se } \quad-c \leq x \leq c \\
|Z| \sqrt{2\left(2-k^{2}\right)} \operatorname{sech}\left(Z \sqrt{2-k^{2}}(x-c+b)\right), \quad \text { se } c<x<+\infty .
\end{array}\right.
$$

Então, para cada $x \in \mathbb{R}$ fixo, deduzimos que

$$
\lim _{Z \rightarrow 0} \Phi_{\omega, Z, c}(x)=0 .
$$

Como a nossa teoria de estabilidade depende da perturbação analítica dos operadores (1.3) e (1.4), quando $Z$ converge para zero, não é desejável então que o perfil dnoidal $\Phi_{\omega, Z, c}$ em (3.8), convirja para a função nula quando $Z \rightarrow 0$.

Assim, da Proposição 3.1 e da Observação 3.2, podemos supor a seguinte

Condição entre o ponto c e o período minimal da onda dnoidal: Para $k \in(0,1)$ e $(c, \omega) \in \mathbb{R}^{+} \times \mathbb{R}^{+}$, supomos que $0<c<\frac{T_{\phi}}{2}=\sqrt{\frac{2-k^{2}}{\omega}} K(k)$.

A figura 3.3 abaixo ilustra o que afirma a Proposição 3.1

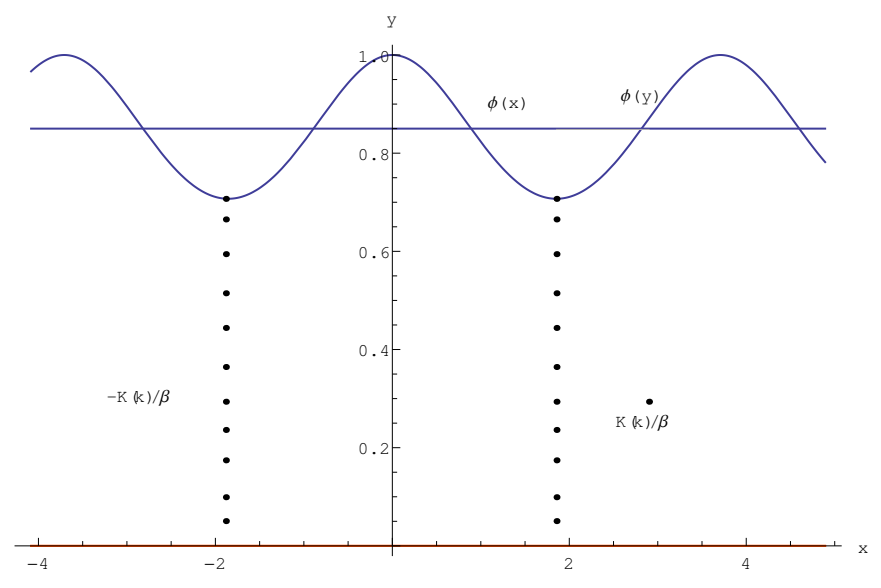

Figura 3.3: Gráfico de $\phi$, para $\frac{T_{\phi}}{2}<c$

Note que da expressão (3.13), deduzida das condições de continuidade (3.9) e salto na derivada (3.10), temos

$$
\lim _{Z \rightarrow 0} \omega\left(1-k^{2}\right)=0
$$

Vamos admitir que os valores admissiveis para $\omega$ estão em um intervalo $I \subset \mathbb{R}$ que não contém o zero. Portanto, devem existir constantes positivas $M$ e $m$, com $m<M$; tais que, se $\omega>0$ é um valor admissível para o modelo (3.8); então $\omega \in I=(m, M)$. Desta maneira, no limite (3.19) devemos ter

$$
\lim _{Z \rightarrow 0} k=1^{-}
$$


Embora esta condição pareça "forçada", ficará claro, nos cálculos a seguir, que é conveniente, porquanto desta maneira; e aproveitando o fato de que $\lim _{k \rightarrow 1^{-}} d n(\gamma ; k)=\operatorname{sech}(\gamma)(\gamma \in \mathbb{R})$, poderemos garantir a valiosa propriedade (3.17).

Em vista de que o período minimal de $\phi$ é $T_{\phi}=2 K(k) \sqrt{\frac{2-k^{2}}{\omega}}$, e levando em consideração a condição anterior sobre $\omega$; notamos que sempre é possível estabelecer a condição entre $c e$ $T_{\phi}$, imposta anteriormente. De fato, já que $K(k) \rightarrow \infty$ quando $k \rightarrow 1^{-}$; se $c>0$ no modelo (3.8) é grande, basta tomar $k$ próximo de $1^{-}$.

Com a seguinte proposição ficará claro o porquê do título desta seção, ou seja, porquê é possível estabelecer um perfil dnoidal entre os pontos de interação $c$ e $-c$ somente quando $Z>0$. Sob a hipótese sobre $\omega$ comentada acima temos o seguinte resultado.

Proposição 3.3 Seja $\Phi_{\omega, Z, c}$ o perfil dnoidal em (3.8), onde $\alpha$ e $\beta$ estão dados como em (3.7). Então, para $\phi=\phi_{\alpha, \beta}$ e $Z>0$ obtemos

$$
\phi^{\prime}\left(c^{-}\right) \Psi_{\omega, b}^{\prime}\left(c^{+}\right)>0, e, \phi^{\prime}\left(-c^{+}\right) \Psi_{\omega,-b}^{\prime}\left(-c^{-}\right)>0 .
$$

Se $Z<0$, então $\Phi_{\omega, Z, c}$ não satisfaz a segunda condição em (3.2).

Demonstração. A condição entre $c$ e $T_{\phi}$, i.e.; $c<\frac{T_{\phi}}{2}$, implica que para $x \in[-c, c]$ temos $-\frac{T_{\phi}}{2}<x<\frac{T_{\phi}}{2}$, ou ainda

$$
-K(k)<x \beta<K(k) .
$$

Portanto,

$$
\begin{aligned}
& \phi^{\prime}(x)<0, \text { para } 0<x<c, \mathrm{e} \\
& \phi^{\prime}(x)>0 \text {, para }-c<x<0 .
\end{aligned}
$$

Decorre disto que $\phi^{\prime}\left(c^{-}\right)<0$ e $\phi^{\prime}\left(-c^{+}\right)>0$. Segue de (3.15) que

$$
\Psi_{\omega, b}^{\prime}\left(c^{+}\right) \phi^{\prime}\left(c^{-}\right) \text {e } \Psi_{\omega,-b}^{\prime}\left(-c^{-}\right) \phi^{\prime}\left(-c^{+}\right)
$$

são positivos.

Por outro lado, por (3.14) sabemos que $b Z>0$. Assuma que $Z<0$, então $b<0$. Seja $\omega>0$ fixo. A condição de salto na derivada (3.10) pode-se escrever como

$$
\sqrt{\omega} \operatorname{sech}(\sqrt{\omega} b) \tanh (-\sqrt{\omega} b)+\frac{\sqrt{\omega} k^{2}}{2-k^{2}} \operatorname{cn}(\beta c) \operatorname{sn}(\beta c)=-Z \operatorname{sech}(\sqrt{\omega} b) .
$$

Note que $\tanh (-\sqrt{\omega} b)>0$. De $(3.21)$ segue

$$
0 \leq \frac{\sqrt{\omega} k^{2}}{2-k^{2}} \operatorname{cn}(\beta c) \operatorname{sn}(\beta c) \leq-Z \operatorname{sech}(\sqrt{\omega} b) .
$$

Tomando o limite quando $Z \rightarrow 0^{-}$em (3.22) temos

$$
\operatorname{sech}(\sqrt{\omega} c) \tanh (\sqrt{\omega} c)=0 .
$$

Aqui foi usado o fato de que $\operatorname{sn}(\gamma ; k) \rightarrow \tanh (\gamma)$, quando $k \rightarrow 1^{-}$. Mas, a equação (3.23) acarreta

$$
\operatorname{sech}(\sqrt{\omega} c)=0 \text { ou } \operatorname{sech}(\sqrt{\omega} c)=1 .
$$

Como $c>0$ é fixo, qualquer das duas últimas igualdades é impossível que aconteça. Logo, a segunda condição em (3.2) não pode ser satisfeita se $b<0$, isto é, se $Z<0$. 
A Proposição 3.3 explica a razão pela qual $Z$ deve ser positivo, se queremos ajustar um perfil $\Phi_{\omega, Z, c}$ com uma função dnoidal entre os pontos $c$ e $-c$.

No nosso estudo de estabilidade não omitiremos a condição $0<c<\frac{T_{\phi}}{2}$, pois ela irá garantir, como veremos, a possibilidade de determinar o índice de Morse do operador $\mathcal{L}_{1, Z, c}$; número que é vital para as nossas conclusões sobre estabilidade.

Embora o ponto $c$ esteja no intervalo $\left(0, \frac{T_{\phi}}{2}\right)$, enfrentamos a questão de saber onde ele está exatamente localizado. A proposição seguinte nos dará um argumento para escolher o ponto $c$ como sendo um ponto específico dentro do intervalo $\left(0, \frac{T_{\phi}}{2}\right)$. Esta escolha é bem feita (não é ambígua) e acarretará boas características sobre o perfil dnoidal $\Phi_{\omega, c, Z}$ em (3.8), no sentido de que muitas contas ficarão relativamente fáceis de fazer.

Proposição 3.4 Seja $\Phi_{\omega, Z, c}$ o perfil dnoidal dado em (3.8), e $Z>0$; com

$$
\alpha=\sqrt{\frac{2 \omega}{2-k^{2}}}, \beta=\sqrt{\frac{\omega}{2-k^{2}}}, \quad \text { e } 0<c<\frac{T_{\phi}}{2} .
$$

Dado que $k \in(0,1)$, então o ponto c deve coincidir com o único ponto em $\left(0, \frac{T_{\phi}}{2}\right)$ para o qual vale a relação $\phi^{\prime \prime}(c)=\Psi_{\omega, b}^{\prime \prime}(c)=0$.

Demonstração. Para $Z>0$ e $0<c<\frac{T_{\phi}}{2}$, sejam $s=\operatorname{sech}(\sqrt{\omega} b)$ e $\gamma=\operatorname{cn}(\beta c)$. De (3.14) sabemos que $b>0$. Portanto, $\tanh (\sqrt{\omega} b)=\sqrt{1-s^{2}}$. Já que $0<c<\frac{T_{\phi}}{2}$, as quantidades $c n(\beta c)$ e $\operatorname{sn}(\beta c)$ são positivas, $\operatorname{logo} s n(\beta c)=\sqrt{1-\gamma^{2}}$.

A condição de salto na derivada, (3.10), se escreve então como

$$
-s \sqrt{1-s^{2}} \sqrt{\omega}+\frac{\sqrt{\omega} k^{2}}{2-k^{2}} \sqrt{2 s^{2}-1+k^{2}-k^{2} s^{2}} \sqrt{1-2 s^{2}+s^{2} k^{2}}=-Z s,
$$

ou equivalentemente,

$$
\sqrt{\omega}\left[\left(2-k^{2}\right) s \sqrt{1-s^{2}}-k^{2} \sqrt{2 s^{2}-1+k^{2}-k^{2} s^{2}} \sqrt{1-2 s^{2}+s^{2} k^{2}}\right]=Z s\left(2-k^{2}\right) .
$$

Observe que $k^{2} \sqrt{2 s^{2}-1+k^{2}-k^{2} s^{2}} \sqrt{1-2 s^{2}+s^{2} k^{2}}<\left(2-k^{2}\right) s \sqrt{1-s^{2}}$, para todo $k \in$ $(0,1)$, então (3.24) é verificada para todo $k \in(0,1)$.

Da condição de continuidade, (3.9), e das identidades elípticas de Jacobi segue-se

$$
s^{2}=\frac{1-k^{2}+k^{2} c n^{2}(\beta c)}{2-k^{2}}=\frac{1-k^{2}+k^{2} \gamma^{2}}{2-k^{2}} .
$$

Agora, isolando $\gamma^{2}$ obtemos

$$
\gamma^{2}=\frac{2 s^{2}-1+k^{2}-k^{2} s^{2}}{k^{2}}
$$

Desta maneira, necessário é que $2 s^{2}-1+k^{2}-k^{2} s^{2} \geq 0 \Leftrightarrow 2 s^{2} \geq \frac{2\left(1-k^{2}\right)}{2-k^{2}}$.

Por outro lado, a identidade

$$
s n^{2}(\beta c)=1-\gamma^{2}=\frac{1-2 s^{2}+k^{2} s^{2}}{k^{2}}
$$

implica que $1-2 s^{2}+k^{2} s^{2} \geq 0 \Leftrightarrow 1+k^{2} s^{2} \geq 2 s^{2}$. Esta análise mostra que a condição de continuidade, (3.9), bem como a condição de salto na derivada (3.10) implicam

$$
\frac{2\left(1-k^{2}\right)}{2-k^{2}} \leq 2 s^{2} \leq 1+k^{2} s^{2}
$$


Já que $k \in(0,1)$, tomando o límite quando $k \rightarrow 0^{+}$em $(3.25)$, deduzimos

$$
\lim _{k \rightarrow 0^{+}} s^{2}=\frac{1}{2},
$$

ou seja, se $k$ varia no intervalo $(0,1)$ todo; então

$$
\operatorname{sech}(\sqrt{\omega} b)=\frac{1}{\sqrt{2}}
$$

Agora, da condição de continuidade (3.9) e de identidades de funções elípticas de Jacobi, obtemos as equivalências

$$
\begin{aligned}
& \frac{1}{\sqrt{2}}=\operatorname{sech}(\sqrt{\omega} b)=\frac{d n(\beta c)}{\sqrt{2-k^{2}}} \Leftrightarrow \\
& 1-\frac{k^{2}}{2}=d n^{2}(\beta c)=1-k^{2}+k^{2} c n^{2}(\beta c) \Leftrightarrow \\
& \frac{1}{2}=c n^{2}(\beta c) .
\end{aligned}
$$

A seguir, defina $f: \mathbb{R} \longrightarrow \mathbb{R}$ por $f(x)=A \operatorname{sech}(B(x+C))$, com $A, B, C \in \mathbb{R}$ e $A B \neq 0$. Então $f^{\prime \prime}(x)=0 \Leftrightarrow \operatorname{sech}^{2}(B(x+C))=\frac{1}{2}$. Ainda mais, a segunda derivada de $\phi$ em (3.8) vem dada por

$$
\phi^{\prime \prime}(x)=\frac{\sqrt{2} \omega k^{2}}{2-k^{2}} d n(\beta x)\left[1-2 c n^{2}(\beta x)\right], \text { para } x \in(-c, c) .
$$

Portanto, como $\omega>0$ obtemos a relação, $\phi^{\prime \prime}(x)=0 \Leftrightarrow c n^{2}(\beta x)=\frac{1}{2}$.

Desta forma, como para $c<x$, temos $\Psi_{\omega, b}(x)=\sqrt{2 \omega} \operatorname{sech}(\sqrt{\omega}(x-c+b))$, então obtemos $\Psi_{\omega, b}^{\prime \prime}\left(c^{+}\right)=0$, pois $\operatorname{sech}^{2}(\sqrt{\omega}(c-c+b))=\frac{1}{2}$. Da mesma forma, obtemos $\phi^{\prime \prime}\left(c^{-}\right)=0$. Claramente, nesta situação temos também que

$$
\Psi_{\omega,-b}^{\prime \prime}\left(-c^{-}\right)=\phi^{\prime \prime}\left(-c^{+}\right)=0 .
$$

Isto finaliza a prova.

Observação 3.5 1. $\Phi_{\omega, Z, c}^{\prime \prime}( \pm c)=0 \Leftrightarrow \Phi_{\omega, Z, c}^{2}( \pm c)=\omega$. Isto é imediato da Proposição 3.4. Concluímos então que o perfil $\Phi_{\omega, Z, c}$ possui segunda derivada contínua na reta toda. Logo, a primeira equação em (3.2), i.e.,

$-\Phi_{\omega, Z, c}^{3}(x)+\omega \Phi_{\omega, Z, c}(x)-\Phi_{\omega, Z, c}^{\prime \prime}(x)=0$ é satisfeita pontualmente para todo $x \in \mathbb{R}-\{ \pm c\}$.

2. $c n(\beta c)=s n(\beta c)=\frac{1}{\sqrt{2}}$. Isto é imediato pela maneira como escolhimos o ponto $c$, $e$ pelas identidades de Jacobi.

3. $\Psi_{\omega, b}^{\prime}\left(c^{+}\right)=-\frac{\omega}{\sqrt{2}}$ e $\Psi_{\omega,-b}^{\prime}\left(-c^{-}\right)=\frac{\omega}{\sqrt{2}}$. Este é um fato que pode ser mostrado de maneira geral. Suponha que $\Phi \in C^{\infty}(\mathbb{R})$, com $\lim _{|x| \rightarrow \infty} \Phi(x)=\lim _{|x| \rightarrow \infty} \Phi^{\prime}(x)=0$, satisfaz a equação

$$
-\Phi^{3}+\omega \Phi-\Phi^{\prime \prime}=0
$$

para $\omega>0$. Então, para $x \in \mathbb{R}$ temos

$$
2 \Phi^{4}(x)+4\left(\Phi^{\prime}(x)\right)^{2}-4 \omega \Phi^{2}(x) \equiv 0,
$$

Se $x_{0} \in \mathbb{R}$ verifica que $\Phi^{2}\left( \pm x_{0}\right)=\omega$; então $\left(\Phi^{\prime}\left( \pm x_{0}\right)\right)^{2}=\frac{\omega^{2}}{2}$. Como caso particular, tome $\Phi \equiv \Phi_{\omega, Z, c}$ e $x_{0}=c$. Devido à condição de salto na derivada (3.10), as derivadas de $\Phi_{\omega, Z, c}$ nos pontos $x= \pm c$ devem ser consideradas unilateralmente. É claro que uma outra forma de provar que $\Psi_{\omega, b}\left(c^{+}\right)=-\frac{\omega}{\sqrt{2}}$ e $\Psi_{\omega,-b}^{\prime}\left(-c^{-}\right)=\frac{\omega}{\sqrt{2}}$ é fazendo a conta diretamente da expressão de $\Phi_{\omega, Z, c}$. 
4. Já que $Z>0$ então $b>0 e$

$$
\operatorname{sech}(\sqrt{\omega} b)=\tanh (\sqrt{\omega} b)=\operatorname{cn}(\beta c)=\operatorname{sn}(\beta c)=\frac{1}{\sqrt{2}} .
$$

Segue que a condição de salto na derivada, (3.10), é equivalente à seguinte equação

$$
Z=\sqrt{2 \omega} \frac{1-k^{2}}{2-k^{2}}
$$

5. Do item 4. anterior, e dado que

$$
\operatorname{sech}(\sqrt{\omega} b)=c n\left(\sqrt{\frac{\omega}{2-k^{2}}} c ; k\right)=\frac{1}{\sqrt{2}} ;
$$

temos, para $k \in(0,1)$,

$$
\left\{\begin{array}{l}
Z=Z(k)=\frac{\sqrt{2}\left(1-k^{2}\right)}{c \sqrt{2-k^{2}}} c n^{-1}\left(\frac{1}{\sqrt{2}} ; k\right), \\
\omega=\omega(k)=\frac{2-k^{2}}{c^{2}}\left[c n^{-1}\left(\frac{1}{\sqrt{2}} ; k\right)\right]^{2} \\
b=b(k)=\frac{c}{\sqrt{2-k^{2}}} \frac{\operatorname{sech}^{-1}\left(\frac{1}{\sqrt{2}}\right)}{c n^{-1}\left(\frac{1}{\sqrt{2}} ; k\right)} .
\end{array}\right.
$$

Fica claro daqui que os parâmetros $\alpha$ e $\beta$, dados em (3.7), podem ser escritos também como função do módulo $k \in(0,1)$. De fato,

$$
\beta=\beta(k)=\frac{1}{c} c n^{-1}\left(\frac{1}{\sqrt{2}} ; k\right) \text { e } \alpha=\alpha(k)=\sqrt{2} \beta(k) .
$$

6. O período da onda dnoidal $\phi$ está dado por

$$
T_{\phi}=\frac{2 K(k)}{\beta}=\frac{2 c K(k)}{c n^{-1}\left(\frac{1}{\sqrt{2}} ; k\right)} .
$$

Portanto, a condição entre o período da onda $\phi, T_{\phi}$, e o ponto $x=c$ (ou seja, $c<\frac{T_{\phi}}{2}$ ); equivale à desigualdade $c n^{-1}\left(\frac{1}{\sqrt{2}} ; k\right)<K(k)$, a qual é verdadeira para todo $k \in(0,1)$, como mostra a figura 3.4 abaixo.

7. Seja $k \in(0,1)$, então as funções $k \mapsto \omega(k)$ e $k \mapsto Z(k)$ são estritamente decrescentes. Além disso,

$$
\omega=\omega(k) \in I_{c}=\left(\omega^{*}, \frac{\pi^{2}}{8 c^{2}}\right)
$$

onde

$$
\omega^{*}=\lim _{k \rightarrow 1^{-}} \omega(k)=\left(\frac{\operatorname{sech}^{-1}\left(\frac{1}{\sqrt{2}}\right)}{c}\right)^{2} ;
$$

$e$

$$
Z=Z(k) \in J_{c}=\left(0, \frac{\pi}{4 c}\right)
$$

sendo

$$
\frac{\pi}{4 c}=\lim _{k \rightarrow 0^{+}} Z(k)
$$




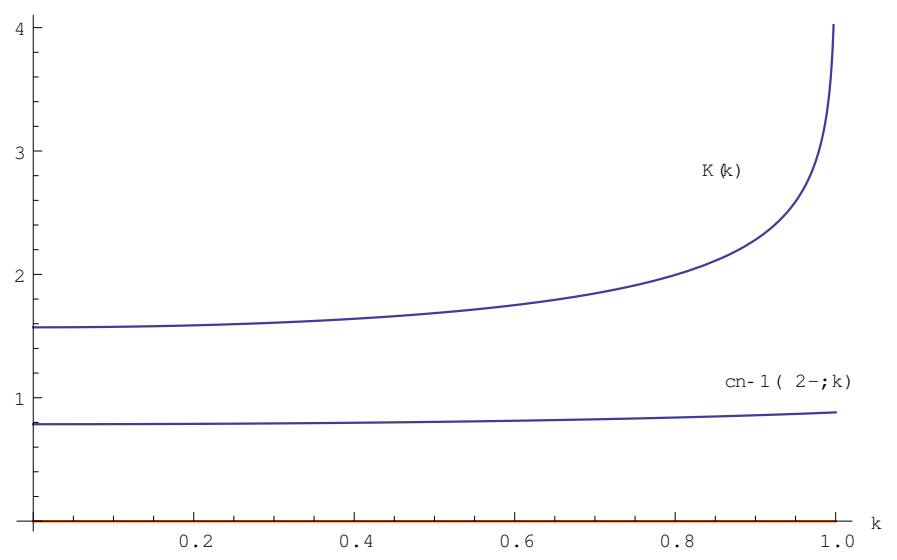

Figura 3.4: Gráficos de $c n^{-1}\left(\frac{1}{\sqrt{2}} ; k\right)$ e $K(k)$

Com a análise feita até aqui, explicaremos a forma em que determinamos o perfil dnoidal $\Phi_{\omega, Z, c}$, como em (3.8), associado à equação NLS- $\delta_{ \pm c}(7)$ e satisfazendo as condições $(10)$.

Defina a função $f:[0,1] \longrightarrow \mathbb{R}$ por

$$
f(k)=\frac{1-k^{2}}{\sqrt{2-k^{2}}} c n^{-1}\left(\frac{1}{\sqrt{2}} ; k\right) .
$$

Dado que $f^{\prime}(k)=\frac{k\left(k^{2}-3\right)}{\sqrt{\left(2-k^{2}\right)^{3}}} c n^{-1}\left(\frac{1}{\sqrt{2}} ; k\right)+\frac{1-k^{2}}{\sqrt{2-k^{2}}} \frac{d}{d k} c n^{-1}\left(\frac{1}{\sqrt{2}} ; k\right)<0$, segue que $f$ é uma função estritamente decrescente. Como $0=f(1)<f(k)<\frac{1}{\sqrt{2}} \frac{\pi}{4}=f(0)$, para todo $k \in(0,1)$; então para $c>0$ fixo, e $Z \in\left(0, \frac{\pi}{4 c}\right)$, o Teorema do valor intermediário garante a existência de um único número $k \in(0,1)$ tal que a primeira expressão em (3.28) é satisfeita. O gráfico de $f$ é mostrado na figura 3.5 abaixo, assim como aquele da função $k \mapsto c^{2} \omega(k)=$ $\left(2-k^{2}\right)\left[c n^{-1}\left(\frac{1}{\sqrt{2}} ; k\right)\right]^{2}$ na figura 3.6 .

Ilustraremos o anterior com um exemplo. Seja $c=0,5$, então os correspondentes intervalos $I_{0,5}$ e $J_{0,5}$ no inciso 7 . da observação $(3.5)$ são $I_{0,5}=\left(4\left[\operatorname{sech}^{-1}\left(\frac{1}{\sqrt{2}}\right)\right]^{2}, \frac{\pi^{2}}{2}\right)$ e $J_{0,5}=\left(0, \frac{\pi}{2}\right)$. Poderemos determinar o perfil dnoidal $\Phi_{\omega, Z, c}$, apenas quando $Z \in J_{0.5}$. Suponhamos que $Z=1,5$; então o único número em $(0,1)$ tal que

$$
1.5=2 \sqrt{2} \frac{1-k^{2}}{\sqrt{2-k^{2}}} c n^{-1}\left(\frac{1}{\sqrt{2}} ; k\right)
$$

é $k \approx 0.258761757$. A partir das fórmulas explícitas dadas em (3.28) é possível calcular exatamente os valores para $\omega$ e para o parâmetro de translação $b$. Da mesma maneira, das fórmulas dadas em (3.29), calculamos $\alpha$ e $\beta$. O gráfico correspondente do perfil $\Phi_{\omega, Z, c}$ para estes valores, é mostrado na figura 3.7

É claro que pela análise feita acima, temos que o perfil dnoidal $\Phi_{\omega, Z, c}$ em (3.8) pode ser obtido como uma função suave do parâmetro $\omega$, ou então como uma função suave do módulo $k$. Mais ainda, já que para todo $k \in(0,1)$ temos $\omega \in I_{c}$ está "longe do zero", obtemos a relação

$$
Z \rightarrow 0^{+} \Leftrightarrow k \rightarrow 1^{-}
$$




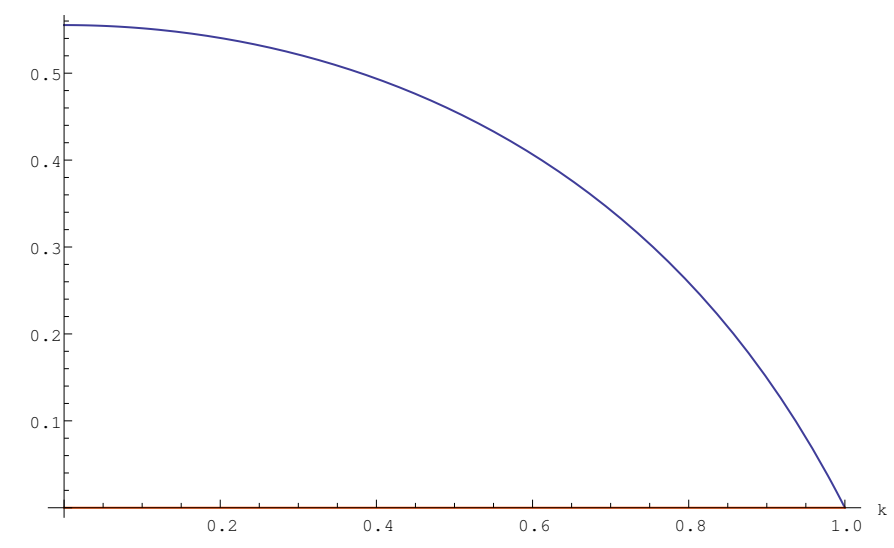

Figura 3.5: Gráfico de $k \in(0,1) \mapsto \frac{1-k^{2}}{\sqrt{2-k^{2}}} c n^{-1}\left(\frac{1}{\sqrt{2}} ; k\right)$

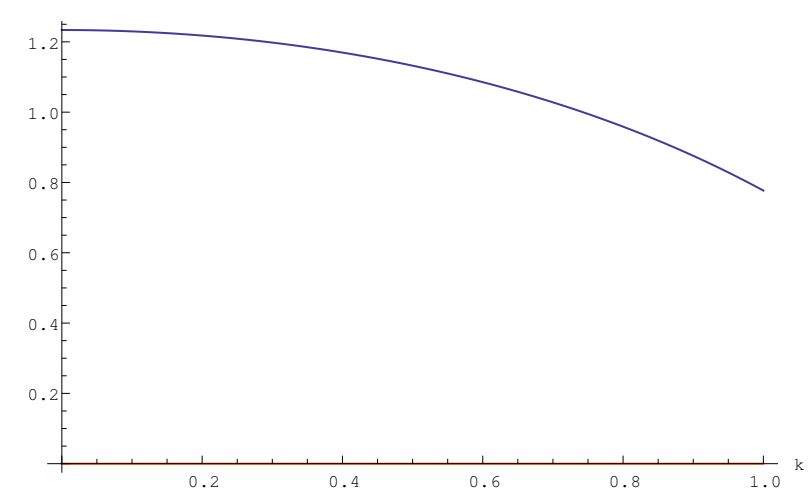

Figura 3.6: Gráfico de $k \in(0,1) \mapsto c^{2} \omega(k)=\left(2-k^{2}\right)\left[c n^{-1}\left(\frac{1}{\sqrt{2}} ; k\right)\right]^{2}$

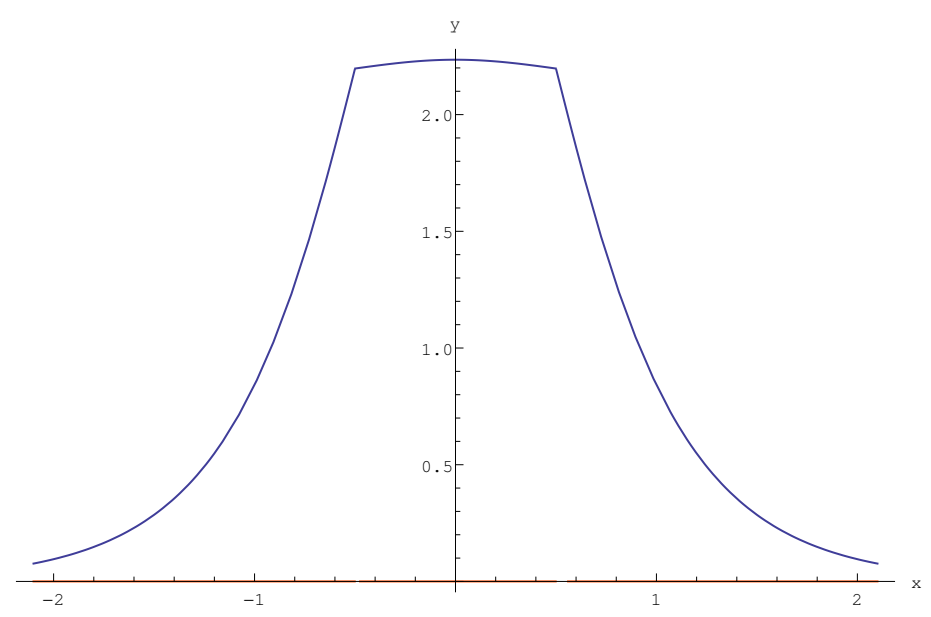

Figura 3.7: Gráfico de $\Phi_{\omega, Z, c}$ para $Z=1,5$ e $c=0.5$ 
Estabelecemos a seguir nosso primeiro Teorema sobre a existência de um perfil periódico de tipo dnoidal para o problema de fronteira (10).

Teorema 3.6 Seja $c>0$ fixo. Para $Z \in\left(0, \frac{\pi}{4 c}\right)$ escolhemos os parâmetros $\omega, b$ e $k \in(0,1)$ tais que $\operatorname{sech}(\sqrt{\omega} b)=\frac{1}{\sqrt{2}}=c n\left(\sqrt{\frac{\omega}{2-k^{2}}} c ; k\right)(\operatorname{ver}(3.28))$. Então, para $\omega \in\left(\frac{1}{c^{2}}\left[\operatorname{sech}^{-1}\left(\frac{1}{\sqrt{2}}\right)\right]^{2}, \frac{\pi^{2}}{8 c^{2}}\right)$ o perfil positivo e par

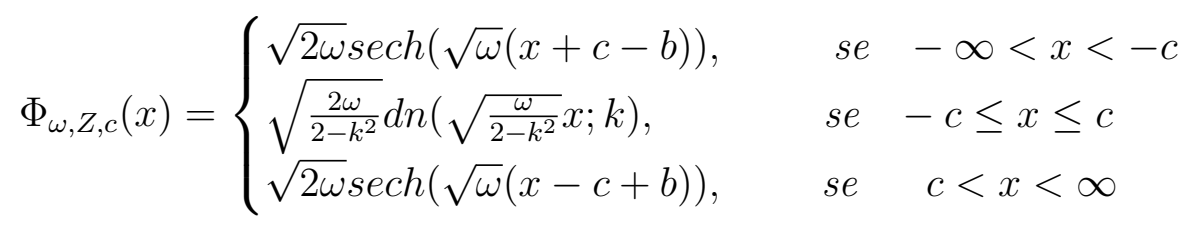

satisfaz as seguintes propriedades:

1. $\Phi_{\omega, Z, c} \in C^{\infty}(\mathbb{R} \backslash\{ \pm c\}) \cap C(\mathbb{R})$,

2. $\Phi_{\omega, Z, c}^{\prime \prime}(x)-\omega \Phi_{\omega, Z, c}(x)+\Phi_{\omega, Z, c}^{3}(x)=0$, para todo $x \in \mathbb{R}$,

3. $\Phi_{\omega, Z, c}^{\prime}\left( \pm c^{+}\right)-\Phi_{\omega, Z, c}^{\prime}\left( \pm c^{-}\right)=-Z \Phi_{\omega, Z, c}( \pm c) \Leftrightarrow Z=\sqrt{2 \omega} \frac{1-k^{2}}{2-k^{2}}$,

4. $\Phi_{\omega, Z, c}^{\prime \prime}( \pm c)=0 \Leftrightarrow \Phi_{\omega, Z, c}( \pm c)=\sqrt{\omega}$,

5. $\lim _{|x| \rightarrow+\infty} \Phi_{\omega, Z, c}(x)=\lim _{|x| \rightarrow+\infty} \Phi_{\omega, Z, c}^{\prime}(x)=0$,

6. $\lim _{Z \rightarrow 0^{+}}|b-c|=\lim _{k \rightarrow 1^{-}}|b(k)-c|=0$,

7. Se definirmos $\Psi(x)=\sqrt{2 \omega^{*}} \operatorname{sech}\left(\sqrt{\omega^{*}} x\right)$, com

$$
\omega^{*}=\left(\frac{\operatorname{sech}^{-1}\left(\frac{1}{\sqrt{2}}\right)}{c}\right)^{2},
$$

então para todo $x \in \mathbb{R}$ temos $\Phi_{\omega, Z, c}(x) \rightarrow \Psi(x)$, quando $Z \rightarrow 0^{+}$. Mais ainda,

$$
\lim _{Z \rightarrow 0^{+}} \Phi_{\omega, Z, c} \equiv \Psi \quad \text { em } \quad H^{1}(\mathbb{R}) .
$$

8. $\Phi_{\omega, Z, c} \in \mathcal{D}\left(-\frac{d^{2}}{d x^{2}}-Z\left[\delta_{c}+\delta_{-c}\right]\right)$.

Demonstração. A prova segue imediatamente da análise feita anteriormente

Cabe destacar aquí a razão pela qual no capítulo 2, onde foi tratado o problema da boa colocação global do problema (2.5), escolhimos $Z$ no intervalo $\left(-\infty, \frac{\pi}{4 c}\right)$ : isto decorre da maneira de escolher o ponto de interação $x=c$ no intervalo $\left(0, \frac{T_{\phi}}{2}\right)$; pois nesta situação, o inciso 5. na obervação 3.5 , implica $0<Z<\frac{\pi}{4 c}$.

$\mathrm{Na}$ figura 3.8 abaixo, mostramos o correspondente diagrama de fase em coordenadas $\left(\Phi, \Phi^{\prime}\right)$, associado ao perfil dnoidal $\Phi \equiv \Phi_{\omega, Z, c}$ dado no Teorema 3.6. Na condição de salto na derivada nos pontos $x= \pm c$ (representada pela reta vertical), consideramos o caso $\omega=2$, e $c=0,63 ; 0,64$ e 0,66 . Note que a trajetória da "parte dnoidal"da $\Phi_{\omega, Z, c}$ está no interior da separatrix determinada pelo soliton "secante hiperbólica", e é percorrida no sentido horáriocomo indicam as setas- (convex profile on $[-c, c]$ ). A diferença das trajetórias do nosso trabalho com aqueles estudados por Jackson\&Weinstein em [30], é que estas últimas estão no sentido anti-horário (concave profile on $[-c, c]$ ). 


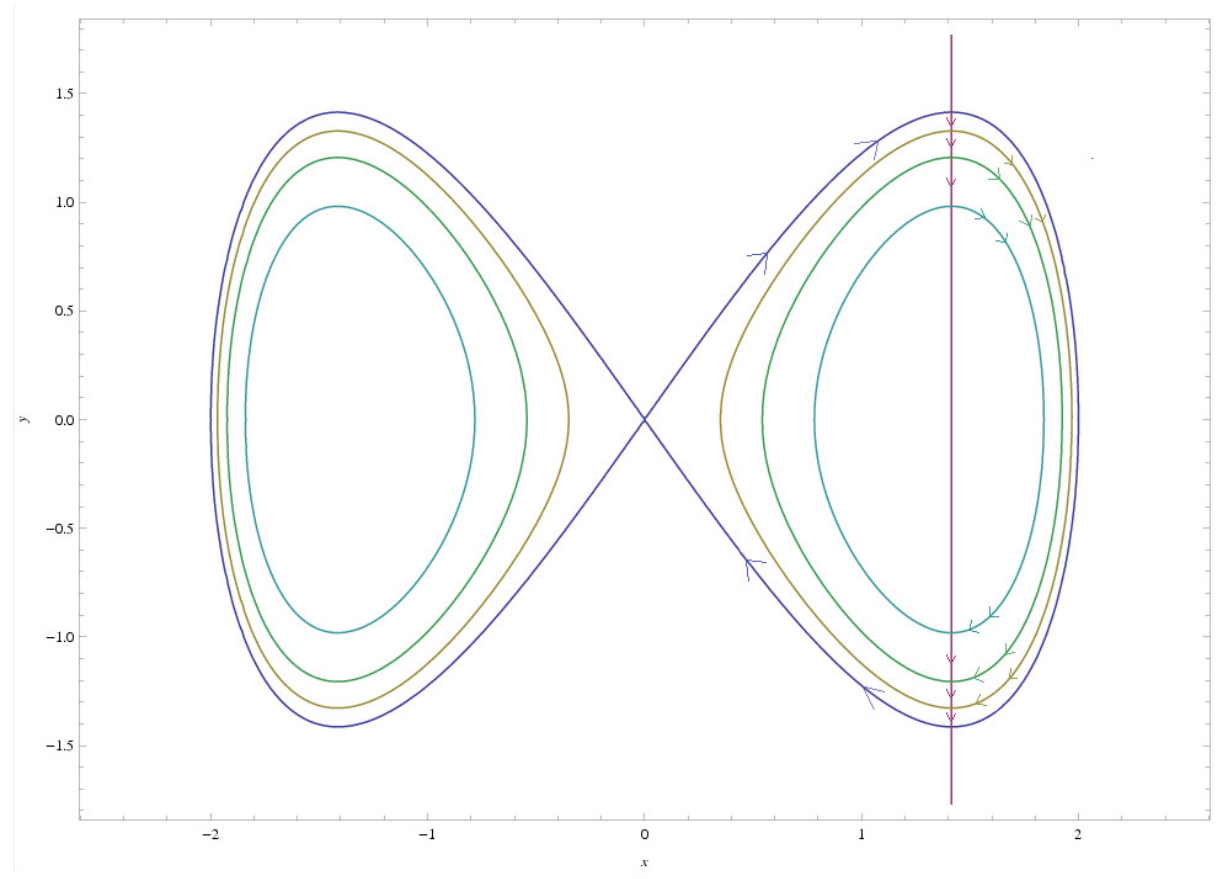

Figura 3.8: Diagrama de fase associado ao perfil dnoidal $\Phi_{\omega, Z, c}$

\subsubsection{Perfil cnoidal para $Z<0$}

Vamos supor agora que entre os pontos $x= \pm c$, temos um perfil cnoidal. Especificamente, uma função do tipo

$$
\tilde{\Phi}_{\omega, Z, c}(x)=\left\{\begin{array}{lll}
\tilde{\Psi}_{-d}(x)=\sqrt{2 \omega} \operatorname{sech}(\sqrt{\omega}(x+c-d)), & \text { se }-\infty<x<-c \\
\tilde{\phi}_{\tilde{\alpha}, \tilde{\beta}, \tilde{\theta}}(x)=\tilde{\alpha} \operatorname{cn}(\tilde{\beta} x+\tilde{\theta} ; k), & \text { se } \quad-c \leq x \leq c \\
\tilde{\Psi}_{d}(x)=\sqrt{2 \omega} \operatorname{sech}(\sqrt{\omega}(x-c+d)), & \text { se } \quad c<x<+\infty
\end{array}\right.
$$

Em uma análise totalmente análoga ao caso do perfil dnoidal, supondo que $\tilde{\phi}_{\tilde{\alpha}, \tilde{\beta}, \tilde{\theta}}$ seja uma solução par da equação elíptica (3.2), devemos ter $\tilde{\theta}=0$ ou $\tilde{\theta}=2 K(k)$ (pois o período da cnoidal $x \mapsto c n(x ; k)$ é $4 K(k))$. Também, para $x \in(-c, c)$, se $\tilde{\alpha} \tilde{\beta} \neq 0$, então

$$
d n^{2}(\tilde{\beta} x+\tilde{\theta} ; k)-\frac{\tilde{\alpha}^{2}}{2 \tilde{\beta}^{2}} c n^{2}(\tilde{\beta} x+\tilde{\theta} ; k)=\frac{\tilde{\beta}^{2}-\omega}{2 \tilde{\beta}^{2}} .
$$

Pelas identidades das funções elípticas de Jacobi se escolhermos, $\frac{\tilde{\alpha}^{2}}{2 \tilde{\beta}^{2}}=k^{2}$ e $\frac{\tilde{\beta}^{2}-\omega}{2 \tilde{\beta}^{2}}=1-k^{2}$, obtemos

$$
\tilde{\alpha}^{2}=\frac{2 \omega}{2 k^{2}-1} k^{2}, \text { and, } \tilde{\beta}^{2}=\frac{\omega}{2 k^{2}-1} \text { e } \tilde{\theta}=0 \text { ou } \tilde{\theta}=2 K(k) .
$$

Dado que $\omega>0$, da expressão de $\tilde{\beta}$ acima deduzimos que $2 k^{2}-1>0 \Leftrightarrow k^{2}>\frac{1}{2}$. Assumiremos, doravante, que $\tilde{\Phi}_{\omega, Z, c}$ é positivo, com $\tilde{\theta}=0$. Então para $\frac{1}{2}<k^{2}<1$ e $\tilde{\theta}=0$, escolhemos

$$
\tilde{\alpha}=\sqrt{\frac{2 \omega}{2 k^{2}-1}} k, \quad \tilde{\beta}=\sqrt{\frac{\omega}{2 k^{2}-1}} .
$$

Nestas circunstâncias, as correspondentes condições de continuidade e salto na derivada que deve satisfazer o perfil $\tilde{\Phi}_{\omega, Z, c}$ em (3.34) são: 
1) Condição de continuidade: O perfil $\tilde{\Phi}_{\omega, Z, c}$ em (3.34) é contínuo em $x= \pm c$, logo

$$
\frac{k c n(\tilde{\beta} c)}{\sqrt{2 k^{2}-1}}=\operatorname{sech}(\sqrt{\omega} d) .
$$

2) Condição de salto na derivada: O perfil $\tilde{\Phi}_{\omega, Z, c}$ verifica a equação

$$
-\sqrt{\omega} \operatorname{sech}(\sqrt{\omega} d) \tanh (\sqrt{\omega} d)+\frac{\sqrt{\omega} k}{2 k^{2}-1} \operatorname{sn}(\tilde{\beta} c) \operatorname{dn}(\tilde{\beta} c)=-Z \operatorname{sech}(\sqrt{\omega} d),
$$

Vamos fazer uma análise sobre a localização do ponto $x=c$. Como $\tilde{\theta}=0$, e o período minimal da onda cnoidal $\tilde{\phi} \equiv \tilde{\phi}_{\tilde{\alpha}, \tilde{\beta}}$, em $(3.34)$, é

$$
T_{\tilde{\phi}}=\frac{4 K(k)}{\tilde{\beta}}=4 K(k) \sqrt{\frac{2 k^{2}-1}{\omega}}, \quad k \in\left(\frac{1}{\sqrt{2}}, 1\right),
$$

então $c \in\left(\frac{3 T_{\tilde{\phi}}}{4}, T_{\tilde{\phi}}\right)$ implica a existência de dois pontos $x \in\left(0, \frac{T_{\tilde{\phi}}}{4}\right)$ e $y \in\left(\frac{3 T_{\tilde{\phi}}}{4}, c\right)$ tais que $\tilde{\phi}(x)=\tilde{\phi}(y)$. Sob a mesma ideia que no caso dnoidal, é desejável que o perfil $\tilde{\Phi}_{\omega, Z, c}$ em (3.34), satisfaça que

$$
\lim _{Z \rightarrow 0} \tilde{\Phi}_{\omega, Z, c}(x)=\tilde{\Psi}(x), \quad \text { para } x \in \mathbb{R} \backslash\{ \pm c\},
$$

sendo $\tilde{\Psi}$ uma função da forma $\tilde{\Psi}(x)=\sqrt{2 \omega^{*}} \operatorname{sech}\left(\sqrt{\omega^{*}} x\right)$, para $\omega^{*}>0$ específico. De forma particular, se isto for possível, deveriamos ter que $\tilde{\phi}(x) \rightarrow \tilde{\Psi}(x)$ e $\tilde{\phi}(y) \rightarrow \tilde{\Psi}(y)$ quando $Z \rightarrow 0$. Mas, como a função secante hiperbólica é estritamente decrescente em $(0, \infty)$, tal coisa não pode acontecer. Mais ainda, isto implica que, com o objetivo de que o limite (3.38) seja obtido; o ponto $c$ não pode pertencer a algum dos intervalos da forma $\left(\frac{3 T_{\tilde{\phi}}}{4}, \frac{5 T_{\tilde{\phi}}}{4}\right) ;\left(\frac{7 T_{\tilde{\phi}}}{4}, \frac{9 T_{\tilde{\phi}}}{4}\right) ;\left(\frac{11 T_{\tilde{\phi}}}{4}, \frac{13 T_{\tilde{\phi}}}{4}\right), \ldots$ etc. Com o objetivo de que o perfil $\tilde{\Phi}_{\omega, Z, c}$ seja positivo, supomos a seguinte

Condição entre o ponto c e o período minimal da onda cnoidal: para $k \in\left(\frac{1}{\sqrt{2}}, 1\right) \mathrm{e}$ $(c, \omega) \in \mathbb{R}^{+} \times \mathbb{R}^{+}$, supomos que $c<\frac{T_{\tilde{\phi}}}{4}=\sqrt{\frac{2 k^{2}-1}{\omega}} K(k)$.

Note que se $c=\frac{T_{\tilde{\Phi}}}{4}$, então $\tilde{\Phi}_{\omega, Z, c}\left(c^{-}\right)=\tilde{\phi}(c)=0$. Portanto, não é possível obter um perfil cnoidal contínuo em $[-c, c]$. Isto segue do fato da função secante hiperbólica ser estritamente positiva na reta. Sob esta condição entre $c$ e $T_{\tilde{\phi}}$ temos a seguinte

Proposição 3.7 Seja $\tilde{\Phi}_{\omega, Z, c}$ o perfil cnoidal dado em (3.34), com $\tilde{\alpha}$ e $\tilde{\beta}$ definidos como em (3.35), e seja $k^{2}>\frac{1}{2}$. Suponha que $0<c<\frac{T_{\tilde{\phi}}}{4}=\sqrt{\frac{2 k^{2}-1}{\omega}} K(k)$.

1) Se $Z>0$ então $d>0$, e não é possivel estabelecer um perfil cnoidal $\tilde{\phi}=\phi_{\tilde{\alpha}, \tilde{\beta}, \tilde{\theta}}$ em $[-c, c]$.

2) Para $Z<0$, se for possivel estabelecer um perfil cnoidal em [-c, c], satisfazendo a condição de salto na derivada (3.37), necessário é que $d>0$. Neste caso temos $\tilde{\Psi}_{-b}^{\prime}\left(-c^{-}\right) \tilde{\phi}^{\prime}\left(-c^{+}\right)>0$ e $\tilde{\phi}^{\prime}\left(c^{-}\right) \tilde{\Psi}_{b}^{\prime}\left(c^{+}\right)>0$. 


\subsection{EXISTÊNCIA DE UM PERFIL PERIÓDICO ENTRE OS PONTOS DE INTERAÇÃO29}

\section{Demonstração.}

1) Outra forma de escrever a condição (3.37) é

$$
\frac{\sqrt{\omega} k \operatorname{sn}(\tilde{\beta} c) d n(\tilde{\beta} c)}{2 k^{2}-1}=\operatorname{sech}(\sqrt{\omega} d)[\sqrt{\omega} \tanh (\sqrt{\omega} d)-Z] .
$$

Já que $0<c<\frac{T_{\tilde{\phi}}}{4}$, então $\operatorname{sn}(\tilde{\beta} c)>0$. Segue da expressão acima que $Z>0$ implica $d>0$.

Por outro lado, sejam $s \equiv \operatorname{sech}(\sqrt{\omega} d)$ e $\gamma \equiv \operatorname{cn}(\tilde{\beta} c)$. Pela condição de continuidade (3.36) temos

$$
\gamma^{2}=\frac{2 k^{2}-1}{k^{2}} s^{2}
$$

$\log 0$

$$
s n^{2}(\tilde{\beta} c)=\frac{k^{2}-\left(2 k^{2}-1\right) s^{2}}{k^{2}}, \quad d n^{2}(\tilde{\beta} c)=1-k^{2}+\left(2 k^{2}-1\right) s^{2} .
$$

Note que para todo $k \in\left(\frac{1}{\sqrt{2}}, 1\right)$ temos $k^{2}-\left(2 k^{2}-1\right) s^{2}, 1-k^{2}+\left(2 k^{2}-1\right) s^{2}>0$. Já que $Z>0$ implica $d>0$, a condição de salto na derivada (3.37) é escrita como

$$
\sqrt{\omega} s \sqrt{1-s^{2}}-\frac{k \sqrt{\omega}}{2 k^{2}-1} \sqrt{k^{2}-\left(2 k^{2}-1\right) s^{2}} \sqrt{1-k^{2}+\left(2 k^{2}-1\right) s^{2}}=Z s,
$$

ou equivalentemente

$\sqrt{\omega}\left[s\left(2 k^{2}-1\right) \sqrt{1-s^{2}}-k \sqrt{k^{2}-\left(2 k^{2}-1\right) s^{2}} \sqrt{1-k^{2}+\left(2 k^{2}-1\right) s^{2}}\right]=Z s\left(2 k^{2}-1\right)>0$.

Mas isto implica

$$
s\left(2 k^{2}-1\right) \sqrt{1-s^{2}}>k \sqrt{k^{2}-\left(2 k^{2}-1\right) s^{2}} \sqrt{1-k^{2}+\left(2 k^{2}-1\right) s^{2}} .
$$

Observe que $k^{2}-\left(2 k^{2}-1\right) s^{2}=k^{2}\left(1-s^{2}\right)+s^{2}\left(1-k^{2}\right)>k^{2}\left(1-s^{2}\right)$, e, $1-k^{2}+$ $\left(2 k^{2}-1\right) s^{2}>s^{2}\left(2 k^{2}-1\right)$, então

$$
\sqrt{k^{2}-\left(2 k^{2}-1\right) s^{2}} \sqrt{1-k^{2}+\left(2 k^{2}-1\right) s^{2}}>s k \sqrt{1-s^{2}} \sqrt{2 k^{2}-1} .
$$

De (3.39) obtemos

$$
\sqrt{2 k^{2}-1}>k^{2} \Leftrightarrow 0>\left(k^{2}-1\right)^{2},
$$

o que claramente é absurdo. Em consequência, se $Z>0$ não é possível estabelecer uma onda cnoidal no intervalo $(-c, c)$.

2) Assuma que $Z<0$ e $b<0$. Segue que $\tanh (\sqrt{\omega} b)<0$ e, com a notação anterior, $|\tanh (\sqrt{\omega} b)|=\sqrt{1-s^{2}}$, portanto $\tanh (\sqrt{\omega} b)=-\sqrt{1-s^{2}}$. A condição de salto na derivada, (3.37), fica então

$\sqrt{\omega}\left[s \sqrt{1-s^{2}}\left(2 k^{2}-1\right)+k \sqrt{k^{2}-\left(2 k^{2}-1\right) s^{2}} \sqrt{1-k^{2}+\left(2 k^{2}-1\right) s^{2}}\right]=-Z s\left(2 k^{2}-1\right)>0$.

Segue, da equação anterior

$$
\sqrt{\omega} s \sqrt{1-s^{2}}\left(2 k^{2}-1\right)<-Z s\left(2 k^{2}-1\right) .
$$

E portanto $\lim _{Z \rightarrow 0^{-}} s=1$, o que é impossível. Desta maneira, se $Z<0$ então $d>0$.

A condição sobre os produtos das derivadas segue do fato de que $d>0$. 
Observação 3.8 Para $\tilde{\Phi}_{\omega, Z, c}$ como em (3.34) obtemos, para $x \in(-c, c), \tilde{\phi}^{\prime \prime}(x)=$ $\tilde{\alpha} \tilde{\beta} c n(\tilde{\beta} x)\left[1-2 d n^{2}(\tilde{\beta} x)\right]$. Portanto, já que $c<\frac{T_{\tilde{\phi}}}{4}$ temos, $\tilde{\phi}^{\prime \prime}(x)=0 \Leftrightarrow d n^{2}(\tilde{\beta} x)=\frac{1}{2}$. Além disto, se $\tilde{\phi}^{\prime \prime}(c)=0$ então, da condição de continuidade $(3.36)$, vale $\operatorname{sech}^{2}(\sqrt{\omega} b)=$ $\frac{1}{2}$. Logo, a função $\tilde{\Psi}_{d}(x)=\sqrt{2 \omega} \operatorname{sech}(\sqrt{\omega}(x-c+d))$ verifica $\tilde{\Psi}_{d}^{\prime \prime}\left(c^{+}\right)=0$.

Embora não exista neste caso cnoidal uma relação básica -ou a priori- similar à Proposição 3.4 como no caso dnoidal que permita dar um argumento para escolher onde está o ponto $c$, estabeleceremos aqui, baseados na observação anterior 3.8, a mesma condição que no caso $Z>0$, a saber: c coincidirá com o único ponto em $\left(0, \frac{T_{\tilde{\phi}}}{4}\right)$ tal que $\operatorname{dn}(\tilde{\beta} c)=\frac{1}{\sqrt{2}}$. O ponto $c \in\left(0, \frac{T_{\tilde{\phi}}}{4}\right)$ é, de fato, o único ponto de inflexão da curva $\tilde{\phi}$ em (3.36). É apropriado fazer as seguintes observações, sendo $\tilde{\Phi}_{\omega, Z, c}$ o perfil cnoidal (3.34) e $Z<0$

Observação 3.9 (a) A condição de salto na derivada, (3.37) é equivalente á equação

$$
Z=-\sqrt{2 \omega} \frac{1-k^{2}}{2 k^{2}-1} .
$$

Então, já que $d n^{-1}(y ; k)$ está bem definida para $\sqrt{1-k^{2}} \leq y<1$, (neste caso particular $\left.y=\frac{1}{\sqrt{2}} e \sqrt{1-k^{2}} \leq \frac{1}{\sqrt{2}} \Leftrightarrow \frac{1}{2} \leq k^{2}\right)$, segue-se de (3.40) que $Z$, $\omega$ e d podem ser determinados como funções do módulo elíptico. Concretamente, para $k \in\left(\frac{1}{\sqrt{2}}, 1\right)$

$$
\left\{\begin{array}{l}
Z=Z(k)=-\frac{\sqrt{2}\left(1-k^{2}\right)}{c \sqrt{2 k^{2}-1}} d n^{-1}\left(\frac{1}{\sqrt{2}} ; k\right), \\
\omega=\omega(k)=\frac{2 k^{2}-1}{c^{2}}\left[d n^{-1}\left(\frac{1}{\sqrt{2}} ; k\right)\right]^{2}, \\
d=d(k)=\frac{c}{\sqrt{2 k^{2}-1}} \frac{\operatorname{sech}^{-1}\left(\frac{1}{\sqrt{2}}\right)}{d n^{-1}\left(\frac{1}{\sqrt{2}} ; k\right)} .
\end{array}\right.
$$

(b) Dado que para todo $k \in\left(\frac{1}{\sqrt{2}}, 1\right)$ temos

$$
d n^{-1}\left(\frac{1}{\sqrt{2}} ; k\right)<K(k), \operatorname{sech}^{-1}\left(\frac{1}{\sqrt{2}}\right)<d n^{-1}\left(\frac{1}{\sqrt{2}} ; k\right)<d n^{-1}\left(\frac{1}{\sqrt{2}} ; \frac{1}{\sqrt{2}}\right),
$$

a partir do inciso (a) anterior resulta que para $\tilde{\beta}=\sqrt{\frac{\omega}{2 k^{2}-1}}$, a condição entre $c$ e o período minimal da onda $\tilde{\phi}$ é sempre obtida, pois $\tilde{\beta} c=d n^{-1}\left(\frac{1}{\sqrt{2}} ; k\right)<K(k)$. Mais ainda,

$$
\omega \in P_{c}=\left(0, \frac{1}{c^{2}}\left[\operatorname{sech}^{-1}\left(\frac{1}{\sqrt{2}}\right)\right]^{2}\right)
$$

(c) Dado que $g(k)=-\frac{1-k^{2}}{\sqrt{2 k^{2}-1}} d n^{-1}\left(\frac{1}{\sqrt{2}} ; k\right)$ é estritamente crescente para $k \in\left(\frac{1}{\sqrt{2}}, 1\right]$, com $g\left(\frac{1}{\sqrt{2}}\right)=-\infty$ e $g(1)=0$, obtemos que para cada

$$
Z \in Q_{c}=(-\infty, 0)
$$


existe um único número $k \in\left(\frac{1}{\sqrt{2}}, 1\right)$ tal que a primeira equação em (3.41) é verificada (Teorema do valor intermediário).

(d) A função $k \in\left(\frac{1}{\sqrt{2}}, 1\right) \mapsto \omega(k)$ é estritamente crescente. Desta análise toda, concluímos que o perfil cnoidal $\tilde{\Phi}_{\omega, Z, c}$ em (3.34) pode ser construído como uma função suave de $\omega$; ou então como uma função suave do módulo $k$.

(e) De (3.41) resulta a vital relação

$$
Z \rightarrow 0^{-} \Leftrightarrow k \rightarrow 1^{-}
$$

Daremos um exemplo da maneira em que pode ser construído um perfil cnoidal $\tilde{\Phi}_{\omega, Z, c}$. Seja $c=0,1$. Então podemos estabelecer nosso perfil cnoidal, por exemplo, para $Z=-1$. De fato, o único número em $k \in\left(\frac{1}{\sqrt{2}}, 1\right)$ que satisfaz a relação

$$
-1=-\frac{\sqrt{2}}{0.1} \frac{1-k^{2}}{\sqrt{2 k^{2}-1}} d n^{-1}\left(\frac{1}{\sqrt{2}} ; k\right)
$$

é aproximadamente $k \approx 0.96394663$. A figura 3.9 mostra este perfil. A figura 3.10 mostra que a função $\omega(k)$ é crescente. Estableceremos a seguir o Teorema sobre a existência de um perfil positivo par, de tipo cnoidal para o problema de fronteira (10), $\operatorname{com} Z<0$. 
Teorema 3.10 Seja $c>0$ fixo. Par $Z \in(-\infty, 0)$ escolhemos os parâmetro $\omega, d e$ $k \in\left(\frac{1}{\sqrt{2}}, 1\right)$ tais que $\operatorname{sech}(\sqrt{\omega} d)=\frac{1}{\sqrt{2}}=d n\left(\sqrt{\frac{\omega}{2 k^{2}-1}} c ; k\right)$ (ver (3.41)). Então, para $\omega \in\left(0, \frac{1}{c^{2}}\left[\operatorname{sech}^{-1}\left(\frac{1}{\sqrt{2}}\right)\right]^{2}\right)$ temos que o perfil positivo e par

$$
\tilde{\Phi}_{\omega, Z, c}(x)=\left\{\begin{array}{llc}
\sqrt{2 \omega} \operatorname{sech}(\sqrt{\omega}(x+c-d)), & \text { se } & -\infty<x<-c \\
\sqrt{\frac{2 \omega}{2 k^{2}-1}} k c n\left(\sqrt{\frac{\omega}{2 k^{2}-1}} x ; k\right), & \text { se } & -c \leq x \leq c \\
\sqrt{2 \omega} \operatorname{sech}(\sqrt{\omega}(x-c+d)), & \text { se } & c<x<\infty
\end{array}\right.
$$

satisfaz:

(a) $\tilde{\Phi}_{\omega, Z, c} \in C^{\infty}(\mathbb{R} \backslash\{ \pm c\}) \cap C(\mathbb{R})$,

(b) $\tilde{\Phi}_{\omega, Z, c}^{\prime \prime}(x)-\omega \tilde{\Phi}_{\omega, Z, c}(x)+\tilde{\Phi}_{\omega, Z, c}^{3}(x)=0$, para todo $x \in \mathbb{R}$,

(c) $\tilde{\Phi}_{\omega, Z, c}^{\prime}\left( \pm c^{+}\right)-\tilde{\Phi}_{\omega, Z, c}^{\prime}\left( \pm c^{-}\right)=-Z \tilde{\Phi}_{\omega, Z, c}( \pm c) \Leftrightarrow Z=-\sqrt{2 \omega} \frac{1-k^{2}}{2 k^{2}-1}$,

(d) $\tilde{\Phi}_{\omega, Z, c}^{\prime \prime}( \pm c)=0 \Leftrightarrow \tilde{\Phi}_{\omega, Z, c}( \pm c)=\sqrt{\omega}$,

(e) $\lim _{|x| \rightarrow+\infty} \tilde{\Phi}_{\omega, Z, c}(x)=\lim _{|x| \rightarrow+\infty} \tilde{\Phi}_{\omega, Z, c}^{\prime}(x)=0$,

(f) $\lim _{Z \rightarrow 0^{-}}|d-c|=\lim _{k \rightarrow 1^{-}}|d(k)-c|=0$,

(g) Se definirmos $\tilde{\Psi}(x)=\sqrt{2 \omega^{*}} \operatorname{sech}\left(\sqrt{\omega^{*}} x\right)$, com

$$
\omega^{*}=\left(\frac{\operatorname{sech}^{-1}\left(\frac{1}{\sqrt{2}}\right)}{c}\right)^{2}
$$

então para todo $x \in \mathbb{R}$ temos $\tilde{\Phi}_{\omega, Z, c}(x) \rightarrow \tilde{\Psi}(x)$, quando $Z \rightarrow 0^{-}$. Além disto,

$$
\lim _{Z \rightarrow 0^{-}} \tilde{\Phi}_{\omega, Z, c} \equiv \Psi \quad \text { em } \quad H^{1}(\mathbb{R}) .
$$

(h) $\tilde{\Phi}_{\omega, Z, c} \in \mathcal{D}\left(-\frac{d^{2}}{d x^{2}}-Z\left[\delta_{c}+\delta_{-c}\right]\right)$.

Notamos que a maneira como fizemos a construção dos perfis dnoidal $\Phi_{\omega, Z, c}$ como no Teorema 3.6, para $Z>0$, e cnoidal $\tilde{\Phi}_{\omega, Z, c}$ como no Teorema 3.10, para $Z<0$ é; em ambos os casos, garantindo que a segunda derivada de tais perfis seja contínua nos pontos $x= \pm c$ (e portanto na reta toda). Esta condição foi inspirada, essencialmente, a partir da Proposição (3.4). Embora particular, esta escolha resulta ser razoavel e essencial para nossa teoria de estabilidade: para ambos os sinais do parâmetro $Z$, o limite do perfil $\Phi_{\omega, Z, c}$ é o mesmo tipo de soliton $\Psi$ !. Além disso, como ficará claro no seguinte capítulo, este fato será essencial para determinar o índice de Morse do operador $\mathcal{L}_{1, Z, c}$.

Concluímos este capítulo com o correspondente diagrama de fase (figura 3.11 abaixo) associado ao perfil cnoidal $\zeta \equiv \tilde{\Phi}_{\omega, Z, c}$ no Teorema 3.10 . Este diagrama, em coordenadas $\left(\zeta, \zeta^{\prime}\right)$, é para o caso particular em que $\omega=2$, e $c=0,63 ; 0.64$ e 0,65 . Notamos que a trajetória da parte "cnoidal"deste diagrama, a qual está no exterior da separatrix determinada pelo soliton de tipo "secante hiperbólica", é percorrida também no sentido horário, como aconteceu no diagrama de fase para o caso dnoidal (ver figura 3.8). Notamos também a paridade deste perfil e a condição de salto na derivada (reta vertical). 
3.1. EXISTENNCIA DE UM PERFIL PERIÓDICO ENTRE OS PONTOS DE INTERAÇÃO33

Figura 3.9: Perfil cnoidal $\tilde{\Phi}_{\omega, Z, c}$ para $Z=-1$ e $c=0.1$ 


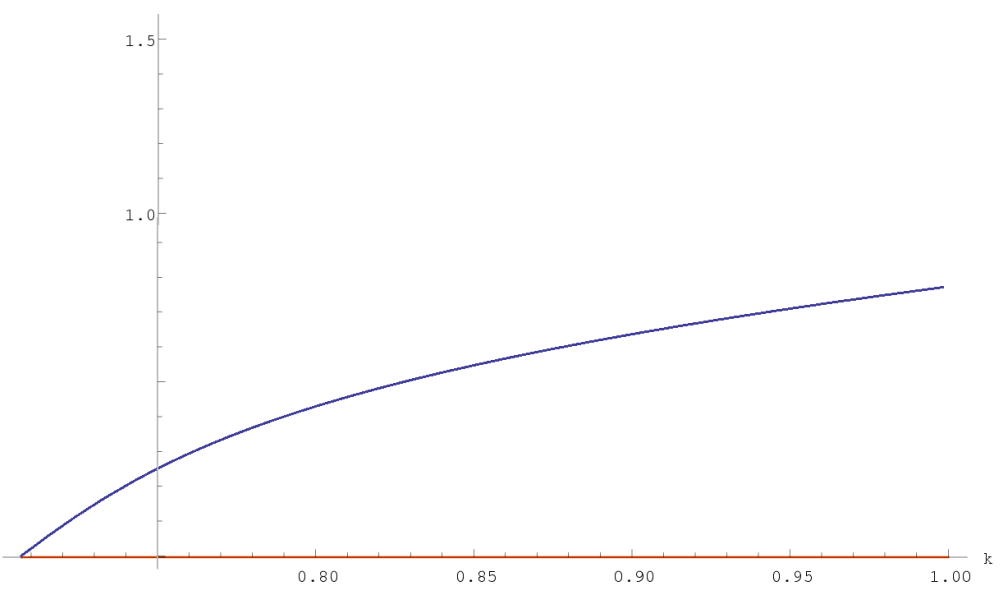

Figura 3.10: Gráfico de $k \in\left(\frac{1}{\sqrt{2}} ; k\right) \rightarrow c^{2} \omega(k)$

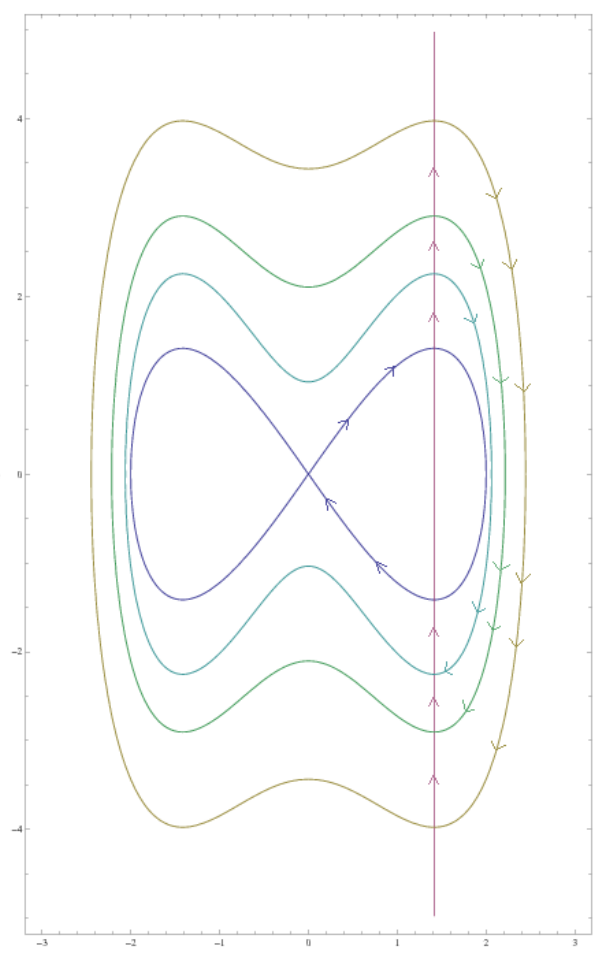

Figura 3.11: Diagrama de fase do perfil cnoidal $\tilde{\Phi}_{\omega, Z, c}$ 


\section{Capítulo 4}

\section{Teoria de Estabilidade}

O objetivo deste capítulo é o de estabelecer a prova do resultado de estabilidade nãolinear estabelecido no Teorema 0.2 da introdução. Nossa estratégia é seguir a teoria geral desenvolvida por Grillakis\&Shatah\&Strauss em [24]. Assim, pelo estabelecido na seção 1.1, nosso intuito é aplicar o Teorema 1.2. Iniciaremos com o estudo de específicas condições espectrais dos operadores $\mathcal{L}_{2, Z, c}$ e $\mathcal{L}_{1, Z, c}$ em (1.4) e (1.3), respectivamente. O uso da teoria de Floquet, da representação de formas bilineares e da teoria de perturbação analítica de operadores serão ferramentas essenciais na nossa análise. $\mathrm{O}$ uso dos projetores de Riesz serão necessários em um argumento de continuação no estudo do índice de Morse para o operador $\mathcal{L}_{1, Z, c}$. A condição de convexidade da aplicação $\omega \rightarrow\left\|\Phi_{\omega, Z, c}\right\|^{2}$ torna-se um problema técnicamente complicado de cálculos devido ao uso de funções de tipo elíptico. Para contornar este problema, foi necessário recorrer a ferramentas de tipo computacional.

\subsection{Análise espectral dos operadores $\mathcal{L}_{1, Z, c}$ e $\mathcal{L}_{2, Z, c}$}

A seguir $\Phi_{Z}$ representará em geral, bem o perfil dnoidal $\Phi_{\omega, Z, c}$ dado no Teorema 3.6, bem o perfil cnoidal $\tilde{\Phi}_{\omega, Z, c}$ no Teorema 3.10 .

Lema 4.1 Seja $Z \neq 0$ e admissivel, no sentido de que $Z \in\left(-\infty, \frac{\pi}{4 c}\right)$. Então,

(1) $\mathcal{L}_{2}=\mathcal{L}_{2, Z, c}$ é um operador não negativo cujo espectro essencial é $[\omega, \infty)$; e com espectro discreto contido em $[0, \omega)$. O zero é um autovalor simples, com autofunção associada $\Phi_{Z}$ e assim $\operatorname{ker}\left(\mathcal{L}_{2}\right)=\left[\Phi_{Z}\right]$.

(2) $\mathcal{L}_{1}=\mathcal{L}_{1, Z, c}$ é um operador com espectro essencial igual a $[\omega,+\infty)$, e espectro discreto em $(-\infty, \omega)$.

Demonstração. Já que $\Phi_{Z}(x) \rightarrow 0$ quando $|x| \rightarrow+\infty$, o Teorema de Weyl's mostra que o espectro essencial dos operadores $\mathcal{L}_{i}$, para $i=1,2$ é como enunciado. Que o espectro discreto seja como afirmado acima, segue de argumentos classicos (ver Berezin\&Shubin [8]).

Agora, de (3.2) segue imediatamente que $\mathcal{L}_{2} \Phi_{Z}=0$. Além do mais, como $\Phi_{Z}>0$, pelo Teorema de Frobenius-Perron, tenemos que $e=\inf \sigma\left(\mathcal{L}_{2}\right)$ é um autovalor simples, portanto deduzimos que $e=0 . \operatorname{Logo}, \operatorname{ker}\left(\mathcal{L}_{2}\right)=\left[\Phi_{Z}\right]$.

Trataremos a seguir um problema delicado, a saber: a estrutura do kernel de $\mathcal{L}_{1, Z, c}$. 
Lema 4.2 Sejam $Z \neq 0$ e $\Phi_{Z}$ definido como no Teorema 3.6 ou no Teorema 3.10. Então $\mathcal{L}_{1, Z, c}$ tem kernel trivial.

Demonstração. Dividiremos a prova para os perfis dnoidal e cnoidal.

a) Caso $Z>0$. Seja $f \in \operatorname{Ker}\left(\mathcal{L}_{1, Z, c}\right)$. Inicialmente, de (3.2) temos $\Phi_{Z} \in C^{\infty}((-\infty,-c) \cup$ $(c,+\infty))$ e para $|x|>c$, vale a relação

$$
-\Phi_{Z}^{\prime \prime \prime}(x)+\left(\omega-3 \Phi_{Z}^{2}\right) \Phi_{Z}^{\prime}(x)=0, \text { e } \lim _{|x| \rightarrow+\infty} \Phi_{Z}^{\prime}(x)=0 .
$$

Portanto, do Theorem 3.6 acima e usando Teorema 3.3 in [8], devem existir constantes $\lambda$ e $\eta$ tais que

$$
f(x)= \begin{cases}\lambda \varphi_{-b}^{\prime}(x), & \text { se } x<-c \\ g(x), & \text { se } x \in[-c, c] \\ \eta \varphi_{b}^{\prime}(x), & \text { se } c<x\end{cases}
$$

onde $\left.g \equiv f\right|_{[-c, c]}$. Agora, dado que para $x \in[-c, c]$ vale $-f^{\prime \prime}(x)+\omega f(x)-$ $3 \phi^{2}(x) f(x)=0$, com

$$
\phi(x)=\sqrt{\frac{2 \omega}{2-k^{2}}} d n\left(\sqrt{\frac{\omega}{2-k^{2}}} x ; k\right)
$$

então para $\beta=\sqrt{\frac{2-k^{2}}{\omega}}$, a função $\psi(x) \equiv f(\beta x)$ satisfaz a seguinte equação de Lamé

$$
L \psi(x) \equiv \psi^{\prime \prime}(x)+\left[k^{2}+4-6 k^{2} s n^{2}(x ; k)\right] \psi=0, \quad x \in\left[-\frac{c}{\beta}, \frac{c}{\beta}\right] .
$$

De (3.2) e do Teorema 3.6 temos $\mathcal{L}_{1, Z, c} \phi^{\prime}(x)=0$ para cada $x \in[-c, c]$. Portanto,

$$
\Lambda_{1}(x) \equiv-\frac{2-k^{2}}{\omega k^{2} \sqrt{2}} \phi^{\prime}(\beta x)=\operatorname{sn}(x ; k) \operatorname{cn}(x ; k), \quad x \in\left[-\frac{c}{\beta}, \frac{c}{\beta}\right]
$$

pertence ao kernel, $\operatorname{Ker}(L)$, de $L$. Segue, da Teoria de Floquet (veja pg. $532 \mathrm{em}$ Angulo\&Ponce [5]) que o problema linear

$$
\left\{\begin{array}{l}
L \Phi(x)=\Phi^{\prime \prime}(x)+\left[k^{2}+4-6 k^{2} s n^{2}(x ; k)\right] \Phi(x)=0, \quad x \in\left[-\frac{c}{\beta}, \frac{c}{\beta}\right] \\
\Phi \in H^{2}\left(-\frac{c}{\beta}, \frac{c}{\beta}\right),
\end{array}\right.
$$

tem por conjunto linearmente independente de soluções em $\left[-\frac{c}{\beta}, \frac{c}{\beta}\right]$, às funções $\left\{\Lambda_{1}, \Lambda_{2}\right\}$. Aqui, se $Z(x)=Z(x ; k)$ representa a funcão Zeta de Jacobi,

$$
Z(x)=\int_{0}^{x}\left[d n^{2}(y ; k)-\frac{E(k)}{K(k)}\right] d y,
$$

a qual é uma funcão periódica com período $2 K(k)$ e ímpar em $x$, temos

$$
\begin{aligned}
\Lambda_{2}(x) & =x\left[2-\frac{2-k^{2}}{1-k^{2}} \frac{E(k)}{K(k}\right] \Lambda_{1}(x) \\
& +\left[\frac{1}{1-k^{2}} d n(x)\left[\operatorname{sn}^{2}(x)-\left(1-k^{2}\right) c n^{2}(x)\right]-\frac{2-k^{2}}{1-k^{2}} Z(x) \Lambda_{1}(x)\right] \\
& \equiv x \gamma \Lambda_{1}(x)+p_{1}(x),
\end{aligned}
$$


com $\gamma<0$ e $p_{1}$ sendo uma funcão par em $\left[-\frac{c}{\beta}, \frac{c}{\beta}\right]$ (na verdade par e períodica na reta, com período $2 K(k)$; se considerarmos $\Lambda_{1}$ definida na reta). Portanto, de (4.2) existem $\alpha, \theta \in \mathbb{R}$ tais que $\psi(y)=\alpha \Lambda_{1}(y)+\theta \Lambda_{2}(y)$, para todo $y \in\left[-\frac{c}{\beta}, \frac{c}{\beta}\right]$. Logo,

$$
f(x)=\alpha \Lambda_{1}(x / \beta)+\theta \Lambda_{2}(x / \beta), \quad \text { para todo } x \in[-c, c] .
$$

Mostraremos que $\alpha=0$. De fato, dado que $f$ é contínua, $\Lambda_{1}$ é ímpar, e que $\Lambda_{2}$ é par, obtemos

$$
\left\{\begin{aligned}
f(c) & =f\left(c^{-}\right)=\alpha \Lambda_{1}\left(c^{-} / \beta\right)+\theta \Lambda_{2}\left(c^{-} / \beta\right) \\
f(-c) & =f\left(-c^{+}\right)=-\alpha \Lambda_{1}\left(c^{-} / \beta\right)+\theta \Lambda_{2}\left(c^{-} / \beta\right) .
\end{aligned}\right.
$$

Desta maneira,

$$
f\left(c^{-}\right)-f\left(-c^{+}\right)=2 \alpha \Lambda_{1}\left(c^{-} / \beta\right) .
$$

A partir de (4.1), obtemos $f\left(c^{-}\right)=f\left(c^{+}\right)=\eta \varphi_{b}^{\prime}\left(c^{+}\right)$e $f\left(-c^{+}\right)=f\left(-c^{-}\right)=$ $\lambda \varphi_{-b}^{\prime}\left(-c^{-}\right)=-\lambda \varphi_{b}^{\prime}\left(c^{+}\right)$. Então, de (4.6), segue

$$
(\eta+\lambda) \varphi_{b}^{\prime}\left(c^{+}\right)=2 \alpha \Lambda_{1}\left(c^{-} / \beta\right) .
$$

Já que $\phi^{\prime \prime}\left(c^{-}\right)=\phi^{\prime \prime}\left(-c^{+}\right)=0$, então $\Lambda_{1}^{\prime}\left(c^{-} / \beta\right)=\Lambda_{1}^{\prime}\left(-c^{+} / \beta\right)=0$. Além do mais, $f^{\prime}\left(c^{+}\right)=\eta \varphi_{b}^{\prime \prime}\left(c^{+}\right)=0=f^{\prime}\left(-c^{-}\right)$. As seguintes relações decorrem do fato de que $f \in \mathcal{D}$ e da paridade de $\Lambda_{2}$

$$
\frac{\theta}{\beta} \Lambda_{2}^{\prime}\left(-c^{+} / \beta\right)=-\frac{\theta}{\beta} \Lambda_{2}^{\prime}\left(c^{-} / \beta\right)=-Z f(-c), \quad \frac{\theta}{\beta} \Lambda_{2}^{\prime}\left(c^{-} / \beta\right)=Z f(c) .
$$

Segue, de (4.6) que

$$
0=Z(f(c)-f(-c))=2 \alpha Z \Lambda_{1}\left(c^{-} / \beta\right) .
$$

então $\alpha=0$. Dessa maneira $f$ em (4.4) se escreve como $f(x)=\theta \Lambda_{2}(x / \beta)$ para cada $x \in[-c, c]$. Mostraremos que $\theta=0$. Para tanto, vejamos que $Z f(c)=$ $-\frac{2}{\beta} f(0)$. De fato, dado que $f \in \operatorname{Ker}\left(\mathcal{L}_{1}\right)$ então

$$
-f^{\prime \prime}+\omega f-3 \Phi_{Z}^{2} f \equiv 0 .
$$

Multiplicando (4.9) por $\Phi_{Z}^{\prime}$ e integrando por partes no intervalo [0,c], deduzimos, pelo Teorema 3.6

$$
-\left.\phi^{\prime} f^{\prime}\right|_{0} ^{c^{-}}+\left.\phi^{\prime \prime} f\right|_{0} ^{c^{-}}=0 .
$$

Agora, da construção do perfil dnoidal, sabemos que $\phi^{\prime}(0)=\phi^{\prime \prime}(c)=0$, então $-\phi^{\prime}\left(c^{-}\right) f^{\prime}\left(c^{-}\right)=\phi^{\prime \prime}(0) f(0)$. Decorre, das relações $\phi^{\prime}\left(c^{-}\right)=-\frac{\omega k^{2}}{\sqrt{2}\left(2-k^{2}\right)}$ e $\phi^{\prime \prime}(0)=$ $-\frac{\omega k^{2}}{2-k^{2}} \sqrt{\frac{2 \omega}{2-k^{2}}}$ que

$$
Z f(c)=f^{\prime}\left(c^{-}\right)=-2 \sqrt{\frac{\omega}{2-k^{2}}} f(0) .
$$

Por outro lado, $Z f(c)=-\frac{2}{\beta} f(0)=-\frac{2}{\beta} \theta \Lambda_{2}(0)=-\frac{2}{\beta} \theta p_{1}(0)=\frac{2}{\beta} \theta$. Consequentemente, $Z f(c)=Z \theta \Lambda_{2}(c / \beta)=\frac{2}{\beta} \theta$. Da condição $f^{\prime}\left(c^{-}\right)=Z f(c)$ deduzimos

$$
\frac{\theta}{\beta} \Lambda_{2}^{\prime}\left(c^{-} / \beta\right)=Z \theta \Lambda_{2}(c / \beta)=\frac{2}{\beta} \theta .
$$


Suponha que $\theta \neq 0$. Então, de (4.12) obtemos $\Lambda_{2}^{\prime}\left(c^{-} / \beta\right)=2$. Logo $2=$ $Z \beta \Lambda_{2}(c / \beta)$. Vejamos que esta relação não é possível. Dos valores especf́icos de $Z \mathrm{e}$ $\beta$ obtemos, $Z \beta=\sqrt{2} \frac{1-k^{2}}{\sqrt{2-k^{2}}}$. Das relações $d n(c / \beta)=\sqrt{\frac{2-k^{2}}{2}}, \frac{d}{d u} d n u=-k^{2}$ snucnu e da igualdade

$$
\left[\frac{d}{d u} d n(u)\right]^{2}=\left(1-d n^{2}(u)\right)\left(d n^{2}(u)-\left(1-k^{2}\right)\right)
$$

segue-se $\operatorname{sn}(c / \beta) \operatorname{cnu}(c / \beta)=\frac{1}{2}$. Então,

$$
\Lambda_{1}(c / \beta)=\frac{1}{2}
$$

Além disso, das identidades elípticas: $k^{2} s n^{2} u+d n^{2} u=1$ e $s n^{2} u+c n^{2} u=1$, obtemos $\operatorname{sn}(c / \beta)=c n(c / \beta)=\frac{1}{\sqrt{2}}$. Logo, da definição de $p_{1}$ em (4.4) deduzimos

$$
p_{1}(c / \beta)=\frac{k^{2}}{2\left(1-k^{2}\right)} \sqrt{\frac{2-k^{2}}{2}}-\frac{2-k^{2}}{2\left(1-k^{2}\right)} Z(c / \beta) .
$$

Ainda mais, da definição de $\Lambda_{2}$ em (4.4) e dado que $2=Z \beta \Lambda_{2}(c / \beta)$ obtemos a igualdade

$$
\frac{1}{\sqrt{2}} \frac{\sqrt{2-k^{2}}}{1-k^{2}}\left[2-\frac{k^{2}}{2}\right]=\frac{c}{2 \beta} \gamma-\frac{2-k^{2}}{2\left(1-k^{2}\right)} Z(c / \beta) .
$$

Agora, de (3.29) na Observação 3.5 temos

$$
Z(c / \beta)=Z\left(c n^{-1}\left(\frac{1}{\sqrt{2}} ; k\right)\right)>0,
$$

pois para todo $k \in(0,1)$ temos $0<c n^{-1}\left(\frac{1}{\sqrt{2}} ; k\right)<\frac{\pi}{2}$, e $Z(x ; k)>0$ se $x \in\left(0, \frac{\pi}{2}\right)$. Já que $\gamma<0$, a equação (4.13) é uma contradição. Logo $\theta=0$ e $f(x)$ é nula em $[-c, c]$. Finalmente, da continuidade de $f$ nos pontos $x= \pm c$ e de (4.1) concluímos que $\eta=\lambda=0$. Portanto, $f(x)=0$ for all $x \in \mathbb{R}$.

b) Caso $Z<0$. A ideia aque é a mesma que no caso $Z>0$. Assim, para $g \in$ $\operatorname{Ker}\left(\mathcal{L}_{1, Z, c}\right)$ existem constantes $\lambda_{1}$ e $\eta_{1}$ tais que

$$
g(x)= \begin{cases}\lambda_{1} \varphi_{-b}^{\prime}(x), & \text { if } x<-c, \\ h(x), & \text { if } x \in[-c, c] \\ \eta_{1} \varphi_{b}^{\prime}(x), & \text { if } c<x\end{cases}
$$

onde $\left.h \equiv g\right|_{[-c, c]}$ é a restrição de $g$ em $[-c, c]$. Dado que, para $x \in[-c, c]$ temos $-g^{\prime \prime}(x)+\omega g(x)-3 \widetilde{\phi}^{2}(x) g(x)=0$, com

$$
\widetilde{\phi}(x)=\sqrt{\frac{2 \omega}{2 k^{2}-1}} k c n\left(\sqrt{\frac{\omega}{2 k^{2}-1}} x ; k\right)
$$

então, para $r=\sqrt{\frac{2 k^{2}-1}{\omega}}$ segue que $\widetilde{\psi}(x) \equiv g(r x)$ satisfaz a seguinte equação de Lamé'

$$
\widetilde{L} \widetilde{\psi}(x) \equiv \widetilde{\psi}^{\prime \prime}(x)+\left[1+4 k^{2}-6 k^{2} s n^{2}(x ; k)\right] \widetilde{\psi}=0, \quad x \in\left[-\frac{c}{\beta}, \frac{c}{\beta}\right] .
$$


Além disso, $\mathcal{L}_{1, Z, c} \widetilde{\phi^{\prime}}(x)=0$ para cada $x \in[-c, c]$. Portanto, a funcção

$$
\Pi_{1}(x) \equiv-\frac{2 k^{2}-1}{\omega k \sqrt{2}} \widetilde{\phi}^{\prime}(r x)=\operatorname{sn}(x ; k) d n(x ; k), \quad x \in\left[-\frac{c}{r}, \frac{c}{r}\right]
$$

é tal que $\Pi_{1} \in \operatorname{Ker}(\widetilde{L})$. Segue, da teoria de Floquet (ver Angulo em [5]) que o problema linear

$$
\left\{\begin{array}{l}
\widetilde{L} \Pi(x)=\Pi^{\prime \prime}(x)+\left[1+4 k^{2}-6 k^{2} s n^{2}(x ; k)\right] \Pi(x)=0, \quad x \in\left[-\frac{c}{r}, \frac{c}{r}\right] \\
\Pi \in H^{2}\left(-\frac{c}{r}, \frac{c}{r}\right),
\end{array}\right.
$$

tem, por conjunto linearmente independente (LI) se solções, às funções $\left\{\Pi_{1}, \Pi_{2}\right\}$ on $\left[-\frac{c}{r}, \frac{c}{r}\right]$, com

$$
\begin{aligned}
\Pi_{2}(x) & =x\left[1+\frac{2-k^{2}}{1-k^{2}} \frac{E(k)}{K(k)}\right] \Pi_{1}(x) \\
& +\left[\frac{c n(x)}{k^{2}-1}\left[k^{4} s n^{2}(x)+\left(1-k^{2}\right) d n^{2}(x)\right]+\frac{2-k^{2}}{1-k^{2}} Z(x) \Pi_{1}(x)\right] \\
& \equiv x \gamma_{2} \Pi_{1}(x)+p_{2}(x),
\end{aligned}
$$

sendo $\gamma_{2}>0$ e $p_{2}$ uma função par em $\left[-\frac{c}{r}, \frac{c}{r}\right]$ (ou par e períodica, com período $4 K(k)$, se considerarmos $\Pi_{1}$ definida na reta toda). Logo, de (4.15) existem $\alpha_{1}, \theta_{1} \in \mathbb{R}$ tais que $g(x)=\alpha_{1} \Pi_{1}(x / r)+\theta_{1} \Pi_{2}(x / r)$ para todo $x \in[-c, c]$.

Mostraremos que $\alpha_{1}=0$. Dado que $g$ é contínua, $\Pi_{1}$ é ímpar e $\Pi_{2}$ é par; obtemos

$$
g\left(c^{-}\right)-g\left(-c^{+}\right)=2 \alpha_{1} \Pi_{1}\left(c^{-} / r\right) .
$$

Por outro lado, como $\widetilde{\phi}^{\prime \prime}\left(c^{-}\right)=\widetilde{\phi}^{\prime \prime}\left(-c^{+}\right)=0=\Pi_{1}^{\prime}\left(c^{-} / r\right)=\Pi_{1}^{\prime}\left(-c^{+} / r\right)$ e $g^{\prime}\left(c^{+}\right)=$ $\eta_{1} \varphi_{b}^{\prime}\left(c^{+}\right)=0=g^{\prime}\left(-c^{-}\right)$, obtemos $g^{\prime}\left(c^{-}\right)=\frac{\theta_{1}}{r} \Pi_{2}^{\prime}\left(c^{-} / r\right)=Z g(c)$ e $g^{\prime}\left(-c^{+}\right)=$ $\frac{\theta_{1}}{r} \Pi_{2}^{\prime}\left(-c^{+} / r\right)=-\frac{\theta_{1}}{r} \Pi_{2}^{\prime}\left(c^{-} / r\right)=-Z g(-c)$. De (4.17) obtemos,

$$
2 \alpha_{1} Z \Pi_{1}\left(c^{-} / r\right)=0 .
$$

Então, $\alpha_{1}=0$. Portanto, $g(x)=\theta_{1} \Pi_{2}(x / r)$ para cada $x \in[-c, c]$. Mostraremos a seguir que $\theta_{1}=0$. Inicialemnte, vejamos que $Z g(c)=-\frac{2 k}{r} g(0)$. De fato, já que $\widetilde{L} g=0$, obtemos, via integração por partes no intervalo $[0, c]$,

$$
-\left.\widetilde{\phi}^{\prime} g^{\prime}\right|_{0} ^{c^{-}}+\left.\widetilde{\phi}^{\prime \prime} g\right|_{0} ^{c^{-}}=0
$$

Desta maneira, dado que pela construção do perfil cnoidal, temos $\widetilde{\phi^{\prime}}(0)=\widetilde{\phi}^{\prime \prime}(c)=$ 0 então $-\widetilde{\phi}^{\prime}\left(c^{-}\right) g^{\prime}\left(c^{-}\right)=\widetilde{\phi}^{\prime \prime}(0) g(0)$. Logo, a partir das relações $\widetilde{\phi}^{\prime}\left(c^{-}\right)=-\frac{\omega}{\sqrt{2}\left(2 k^{2}-1\right)}$ e $\widetilde{\phi}^{\prime \prime}(0)=-\frac{\omega k}{2 k^{2}-1} \sqrt{\frac{2 \omega}{2 k^{2}-1}}$ deduzimos

$$
Z g(c)=g^{\prime}\left(c^{-}\right)=-2 k \sqrt{\frac{\omega}{2 k^{2}-1}} g(0) .
$$

Observe que $Z g(c)=-\frac{2 k}{r} g(0)=-\frac{2 k}{r} \theta_{1} \Pi_{2}(0)=-\frac{2 k}{r} \theta_{1} p_{2}(0)=\frac{2 k}{r} \theta_{1}$. Então $Z g(c)=Z \theta_{1} \Pi_{2}(c / r)=\frac{2 k}{r} \theta_{1}$. A condição $g\left(c^{-}\right)=Z g(c)$ implica

$$
\frac{\theta_{1}}{r} \Pi_{2}^{\prime}\left(c^{-} / r\right)=\frac{2 k}{r} \theta_{1} \text {. }
$$


Suponha que $\theta_{1} \neq 0$. Então, de (4.20) segue $\Pi_{2}^{\prime}\left(c^{-} / r\right)=2 k$. Logo então $2 k=$ $Z r \Pi_{2}(c / r)$. Vejamos que esta última equação não pode acontecer. Inicialmente, das expressões específicas para $Z$ e $r$, temos $Z r=-\sqrt{2} \frac{1-k^{2}}{\sqrt{2 k^{2}-1}}$. Por outro lado, as relações

$$
d n(c / r)=\frac{1}{\sqrt{2}}, \quad s n(c / r)=\frac{1}{\sqrt{2} k}, \quad c n(c / r)=\frac{1}{k} \sqrt{\frac{2 k^{2}-1}{2}},
$$

implicam $\Pi_{1}(c / r)=\frac{1}{2 k} \mathrm{e}$,

$$
p_{2}(c / r)=-\frac{k}{2\left(k^{2}-1\right)} \sqrt{\frac{2 k^{2}-1}{2}}+\frac{2-k^{2}}{2 k\left(1-k^{2}\right)} Z(c / r) .
$$

Desta maneira, da definição de $\Pi_{2}$ in $(4.16)$ e de $2 k=Z r \Pi_{2}(c / r)$ concluímos a igualdade

$$
-\frac{2 k \sqrt{2 k^{2}-1}}{\sqrt{2}\left(1-k^{2}\right)}-\frac{k \sqrt{2 k^{2}-1}}{2 \sqrt{2}\left(1-k^{2}\right)}=\frac{c}{2 k r} \gamma_{2}+\frac{2-k^{2}}{2 k\left(1-k^{2}\right)} Z(c / r) .
$$

Agora, da Observação 3.9 segue

$$
Z(c / r)=Z\left(d n^{-1}\left(\frac{1}{\sqrt{2}} ; k\right)\right)>0,
$$

pois para todo $k \in\left(\frac{1}{\sqrt{2}}, 1\right)$ temos $0<d n^{-1}\left(\frac{1}{\sqrt{2}} ; k\right)<\frac{\pi}{2}$, e $0<Z(x ; k)$ se $x \in\left(0, \frac{\pi}{2}\right)$. Já que $\gamma_{2}>0$, a equação (4.21) é absurda.

Portanto, $\theta_{1}=0$ e então $g(x)=0$ para cada $x \in \mathbb{R}$. Com isto termina a prova.

A seguinte proposição será usada depois, e descreve as autofunções associadas aos autovalores simples do operador $\mathcal{L}_{1}$.

Proposição 4.3 Seja $Z \neq 0$. Se $\lambda$ é um autovalor simples do operador $\mathcal{L}_{1}$, então as correspondentes autofunções associadas a $\lambda$ são ou pares ou impares.

Demonstração. Seja $v \in D\left(\mathcal{L}_{1}\right)-\{0\}$ tal que $\mathcal{L}_{1} v=\lambda v$. Então, já que $\Phi_{Z}$ é par, se definirmos a função $\zeta$ por $\zeta(x) \equiv v(-x)$, obtemos $\mathcal{L}_{1} \zeta(x)=\lambda \zeta(x)$ para $x \neq \pm c$. A simplicidade de $\lambda$ implica a existência de $\beta \in \mathbb{R}$ tal que $v(x)=\beta v(-x)$, para $x \in \mathbb{R}$. Daqui, se $v(c) \neq 0$ então $\beta=1$ e $v$ é par. Por outro lado, se $v(c)=0$, de (4.24) temos $v \in H^{2}(0,+\infty)$ e $v^{\prime}(x)$ existe para $x>0$. Devemos ter então $v^{\prime}(c)=-\beta v^{\prime}(c)$, e pelo princípio de unicidade de Cauchy, $v^{\prime}(c) \neq 0$ (de outra forma teriamos $v \equiv 0 \mathrm{em}$ $(0, \infty))$. Portanto $\beta=-1$, e então $v(x)=-v(-x)$ para todo $x$. Logo $v$ é uma função ímpar.

\section{2 Índice de Morse para $\mathcal{L}_{1, Z, c}$}

Obter o número de autovalores negativos do operador $\mathcal{L}_{1, Z, c}$ é de vital importância para nosso resultado de estabilidade orbital dos perfis dnoidal e cnoidal estabelecidos no capítulo 3. Com este objetivo, usaremos nesta seção a teoria de perturbação analítica para operadores lineares (ver Kato [31]), bem como alguns argumentos encontrados em Le Coz et al. [35]. O trabalho será dividido em vários passos: 
(I) Da análise feita no Capítulo 3 segue-se para $\Phi_{Z}$, denotando bem o perfil dnoidal no Teorema 3.6 ou então o perfil cnoidal no Teorema 3.10, a convergência

$$
\lim _{Z \rightarrow 0} \Phi_{Z} \equiv \Psi \quad \text { em } H^{1}(\mathbb{R}) .
$$

Aqui, $\Psi(x) \equiv \sqrt{2 \omega^{*}} \operatorname{sech}\left(\sqrt{\omega^{*}} x\right), \omega^{*}=\left(\frac{\operatorname{sech}^{-1}\left(\frac{1}{\sqrt{2}}\right)}{c}\right)^{2}, x \in \mathbb{R}$.

(II) O operador linear $\mathcal{L}_{1, Z, c}$ é o operador autoadjunto definido em $L^{2}(\mathbb{R})$ associado à seguinte forma bilinear, definida para $u, v \in H^{1}(\mathbb{R})$, como

$$
t_{Z}[f, g]=-Z[f(c) g(c)+f(-c) g(-c)]+\left\langle f^{\prime}, g^{\prime}\right\rangle_{2}+\omega\langle f, g\rangle_{2}-\left\langle 3 \Phi_{Z}^{2} f, g\right\rangle_{2}
$$

De fato, das propriedades dos perfis $\Phi_{Z}$ e da velocidade $\omega$, não é defícil verificar que $t_{Z}$ é simétrica, limitada inferiormente e fechada. De fato, dos Teoremas 3.6 e 3.10 temos:

$$
\begin{aligned}
& \Phi_{Z}^{2} \leq \frac{2}{c^{2}}\left(\operatorname{sech}^{-1}\left(\frac{1}{\sqrt{2}}\right)\right)^{2}, \quad \text { se } Z>0 ; \text { e } \\
& \Phi_{Z}^{2} \leq \frac{2}{c^{2}}\left(d n^{-1}\left(\frac{1}{\sqrt{2}} ; \frac{1}{\sqrt{2}}\right)\right)^{2}, \quad \text { se } Z<0 .
\end{aligned}
$$

Assim, como o perador $\mathcal{L}_{2, Z, c}$ é positivo segue-se

$$
t_{Z}[f, f] \geq-\int_{\mathbb{R}} 2 \Phi_{Z}^{2} f^{2} d x \geq-\frac{4}{c^{2}}\left(d n^{-1}\left(\frac{1}{\sqrt{2}} ; \frac{1}{\sqrt{2}}\right)\right)^{2}\|f\|_{2}^{2},
$$

para todo $Z$ admissível e $f \in H^{1}(\mathbb{R})$. Segue então da teoria de representação de formas bilineares para operadores, [31, VI. Seção 2.1, Teorema 2.7], que existe um operador autoadjunto $\widetilde{\mathcal{L}_{1, Z}}: D\left(\widetilde{\mathcal{L}_{1, Z}}\right) \subset L^{2}(\mathbb{R}) \rightarrow L^{2}(\mathbb{R})$ tal que

$$
D\left(\widetilde{\mathcal{L}_{1, Z}}\right)=\left\{v \in H^{1}(\mathbb{R}): \exists w \in L^{2}(\mathbb{R}) \text { tal que } \forall z \in H^{1}(\mathbb{R}) ; t_{Z}[v, z]=\langle w, z\rangle\right\},
$$

e para $v \in D\left(\widetilde{\mathcal{L}_{1, Z}}\right)$ definimos $\widetilde{\mathcal{L}_{1, Z}} v=w$, onde $w$ é a única função de $L^{2}(\mathbb{R})$ que satisfaz

$$
t_{Z}[v, z]=\left\langle\widetilde{\mathcal{L}_{1, Z}} v, z\right\rangle, \quad \text { para todo } z \in H^{1}(\mathbb{R}) .
$$

A seguir, descrevemos explícitamente o operador autoadjunto $\widetilde{\mathcal{L}_{1, Z}}$.

Proposição 4.4 O dominio do operador $\widetilde{\mathcal{L}_{1, Z}}$ definido em $L^{2}(\mathbb{R})$ é

$$
D_{Z}=\left\{\zeta \in H^{1}(\mathbb{R}) \cap H^{2}(\mathbb{R}-\{ \pm c\}): \zeta^{\prime}\left( \pm c^{+}\right)-\zeta^{\prime}\left( \pm c^{-}\right)=-Z \zeta( \pm c)\right\}
$$

e para $v \in D_{Z}$ obtemos

$$
\widetilde{\mathcal{L}_{1, Z}} v=-\frac{d^{2}}{d x^{2}} v+\omega v-3 \Phi_{Z}^{2} v
$$

Demonstração. Considere $t_{Z}=\mathcal{Q}_{Z}^{1}+\mathcal{Q}_{\omega}^{1}$, com $\mathcal{Q}_{Z}^{1}: H^{1} \times H^{1} \rightarrow \mathbb{R}$, e $\mathcal{Q}_{\omega}^{1}$ : $L^{2} \times L^{2} \rightarrow \mathbb{R}$ definidos por

$$
\mathcal{Q}_{Z}^{1}(v, z)=\left\langle v_{x}, z_{x}\right\rangle-Z[v(c) z(c)+v(-c) z(-c)], \quad \mathcal{Q}_{\omega}^{1}(v, z)=\omega\langle v, z\rangle-3\left\langle\Phi_{Z}^{2} v, z\right\rangle .
$$


Denotemos por $\mathcal{T}_{1}$ (resp. $\mathcal{T}_{2}$ ) o operador autoadjunto em $L^{2}$ associado a $\mathcal{Q}_{Z}^{1}$ (resp. $\left.\mathcal{Q}_{\omega}^{1}\right)$. Portanto, $D\left(\mathcal{T}_{1}\right)=D\left(\widetilde{\mathcal{L}_{1, Z}}\right)\left(D\left(\mathcal{T}_{2}\right)=L_{\text {per }}^{2}\right)$.

A seguir mostraremos que $\mathcal{T}_{1}$ é uma extensão autoadjunta do operador simétrico $-A_{Z}$ definido em (6.20)-Apêndice. De fato, seja $v \in H^{1}(\mathbb{R}) \cap H^{2}(\mathbb{R}-\{c\})$ tal que

$$
v^{\prime}(c+)-v^{\prime}(c-)=-Z v(c), \text { e } v(-c)=0,
$$

e defina $w \equiv-v_{x x} \in L^{2}(\mathbb{R})$. Então, para cada $z \in H^{1}$ temos

$$
\begin{aligned}
\mathcal{Q}_{Z}^{1}(v, z) & =(w, z)+z(-c)\left[v^{\prime}(-c-)-v^{\prime}(-c+)\right]-z(c)\left[v^{\prime}(c+)-v^{\prime}(c-)\right] \\
& -Z[v(c) z(c)+v(-c) z(-c)] \\
& =(w, z)+z(-c)[0]-z(c)[-Z v(c)]-Z[v(c) z(c)]=(w, z)
\end{aligned}
$$

Portanto, $v \in D\left(\mathcal{T}_{1}\right)$ e $\mathcal{T}_{1} v=w=-\frac{d^{2}}{d x^{2}} v$. Logo, $-A_{Z} \subset \mathcal{T}_{1}$. Desta forma, usando a Proposição 6.4 no Apêndice, existe $\gamma \in \mathbb{R}$ tal que $D\left(\mathcal{T}_{1}\right)=D\left(-\Delta_{-Z, \gamma}\right)$, o que prova nossa afirmação.

Devemos mostrar agora que $\gamma=-Z$. Tome $v \in D\left(\mathcal{T}_{1}\right)$ com $v(-c) \neq 0$, então

$$
\left(\mathcal{T}_{1} v, v\right)=\gamma|v(-c)|^{2}-Z|v(c)|^{2}+\int\left|v_{x}\right|^{2} d x
$$

deve ser igual a $\mathcal{Q}_{Z}^{1}(v, v)=\int\left|v_{x}\right|^{2} d x-Z\left[|v(c)|^{2}+|v(-c)|^{2}\right]$. Portanto $\beta=-Z$, o que prova Proposição.

Pela proposição (4.4) podemos tirar a "til"em $\widetilde{\mathcal{L}_{1, Z}}$ e considerar o operador $\mathcal{L}_{1, Z}$ como foi definido em (1.4).

(III) O lema a seguir mostra a analiticidade da família de operadores autoadjuntos $\left\{\mathcal{L}_{1, Z}\right\}_{Z}$, indexada pelo parâmetro $Z$, no sentido $(B)$ de Kato. Este fato permitirá desenvolver em "série de Taylor", tanto a aplicação $Z \longmapsto \Phi_{Z}$ quanto a função autovalor negativo, necessária para determinar o número de autovalores negativos de $\mathcal{L}_{1, Z, c}$, dependendo do sinal de $Z$.

Lema 4.5 Como uma função de $Z,\left\{\mathcal{L}_{1, Z}\right\}_{Z}$ é uma família analítica de operadores autoadjuntos no sentido (B) de Kato.

Demonstração. Do Teorema [31, 4.2 Capítulo VII-4.2, pg. 395] é suficiente provar que a familia de formas bilineares $\left(t_{Z}\right)_{Z}$ definida em (4.22), é uma família real-analítica de tipo $(B)$. De fato, esta forma é limitada por baixo e fechada pelo feito anteriormente. A decomposição de $t_{Z}$ em $\mathcal{Q}_{Z}^{1}$ e $\mathcal{Q}_{\omega}^{1}$, implica que $Z \rightarrow\left\langle t_{Z} v, v\right\rangle$ é analítica.

Observação 4.6 É possível estabelecer uma afirmação similar à Proposição 4.4 e ao Lemma 4.5 para o operador $\mathcal{L}_{2, Z}$ definido em (1.3).

Pelo Teorema 3.6 sabemos que $\Phi_{Z} \equiv \Phi_{\omega, Z, c}$ converge em $H^{1}(\mathbb{R})$ à função

$$
\Psi(x)=\sqrt{2 \omega^{*}} \operatorname{sech}\left(\sqrt{\omega^{*}} x\right) .
$$

Desta maneira, consideremos o operador $\mathcal{L}_{1, \Psi}: H^{2}(\mathbb{R}) \longrightarrow L^{2}(\mathbb{R})$ definido por

$$
\mathcal{L}_{\Psi}=-\frac{d^{2}}{d x^{2}}+\omega^{*}-3 \Psi^{2}
$$

Então, de uma imediata aplicação da teoria de Oscilação de Sturm-Liouville, obtemos os seguintes resultados para o operador $\mathcal{L}_{\Psi}$ definido acima: 
Lema 4.7 $O$ operador $\mathcal{L}_{\Psi}$ tem exatamente um único autovalor negativo e simples. O segundo autovalor é o zero, que é isolado e simples. Além disso, $\operatorname{ker}\left(\mathcal{L}_{\Psi}\right)=\left[\Psi^{\prime}\right]$ e o resto do espectro é positivo.

Combinando os Lemas 4.5 e 4.7, podemos aplicar o Teorema de Kato-Rellich (ver Reed\&Simon [37]) para obter o seguinte resultado.

Proposição 4.8 Existem $Z_{0}>0$ e duas funções analíticas; $\Lambda:\left(-Z_{0}, Z_{0}\right) \longrightarrow \mathbb{R}$ e $\Omega:\left(-Z_{0}, Z_{0}\right) \longrightarrow L^{2}(\mathbb{R})$ tais que

i. $\Lambda(0)=0$ e $\Omega(0)=\frac{d}{d x} \Psi$,

ii. Para cada $Z \in\left(-Z_{0}, Z_{0}\right) ; \Lambda(Z)$ é o segundo autovalor negativo de $\mathcal{L}_{1, Z}$, sendo $\Omega(Z)$ o seu correspondente autovetor associado. Além disso, $\Lambda(Z)$ é simples e isolado.

iii. $Z_{0}$ pode ser escolhido o "suficientemente pequeno"para garantir que, com exceção dos dois primeiros autovalores (negativos) de $\mathcal{L}_{1, z}$; o resto do espectro de $\mathcal{L}_{1, Z}$ seja positivo.

Demonstração. Do Lema 4.7, separemos o espectro, $\sigma\left(\mathcal{L}_{1, \Psi}\right)$, do operador $\mathcal{L}_{1, \Psi}$ em duas partes $\sigma_{0}=\left\{\tau_{0}, 0\right\}$ e $\sigma_{1}$ por uma curva fechada $\Gamma$ (por exemplo um círculo) tal que $\sigma_{0}$ pertença à parte interior do plano (domínio) delimitada pela curva $\Gamma$ e tal que $\sigma_{1}$ no outro domínio (exterior) a $\Gamma$ (note que $\sigma_{1} \subset(a,+\infty)$ for $a>0)$. Do Lema 4.5 segue que $\Gamma \subset \rho\left(\mathcal{L}_{1, Z}\right)$ para $|Z|$ suficientemente pequeno, e $\sigma\left(\mathcal{L}_{1, Z}\right)$ é igualmente separado por $\Gamma$ em duas partes: a parte de $\sigma\left(\mathcal{L}_{1, Z}\right)$ dentro de $\Gamma$ consta de un número finito de autovalores, com multiplicidade algébrica total igual a 2 (dois) (lembre que o zero não é um autovalor de $\mathcal{L}_{1, Z}$ ). Agora, para $\epsilon$ pequeno consideremos os contornos

$$
\Gamma_{1}\left(\tau_{0}\right)=\left\{z \in \mathbb{C}:\left|z-\tau_{0}\right|<\epsilon\right\} \text { e } \Gamma_{2}(0)=\{z \in \mathbb{C}:|z|<\epsilon\},
$$

de tal maneira que $\Gamma_{1}\left(\tau_{0}\right) \cap \Gamma_{2}(0)=\emptyset$, e que os únicos pontos $\sigma\left(\mathcal{L}_{1, \Psi}\right)$ no domínio interior de $\Gamma_{i}$ sejam $\tau_{0}$ and 0 . Portanto, da não degeneracidade de $\tau_{0}$ e 0 , obtemos, do Teorema de Kato-Rellich, a existência de duas funções analíticas, $\Lambda$ e $\Omega$, definidas em uma vizinhança do zero; tais que os itens $i$., $i i$. e iii. acima são verificados. Isto completa a prova do Lema.

Estudaremos agora a maneira como é perturbado o segundo autovalor negativo de $\mathcal{L}_{1, Z}$, a saber, queremos determinar o sinal de $\Lambda(Z)$ para $|Z|$ pequeno.

Teorema 4.9 Existe $Z_{1}$, com $0<Z_{1}<Z_{0}$ tal que $\Lambda(Z)>0$ para qualquer $Z \in\left(-Z_{1}, 0\right)$ e $\Lambda(Z)<0$ para qualquer $Z \in\left(0, Z_{1}\right)$. Portanto, para $Z$ positivo pequeno (próximo do zero) $\mathcal{L}_{1, Z}$ tem exatamente dois autovalores negativos; e para $Z$ negativo, próximo do zero, $\mathcal{L}_{1, Z}$ tem exatamente um autovalor negativo.

Demonstração. A analiticidade de $\Lambda$, para $|Z|$ pequeno; permite desenvolver $\Lambda(Z)$ en série de Taylor em uma vizinhança do zero. Portanto, da fórmula de Taylor temos

$$
\Lambda(Z)=\beta Z+O\left(Z^{2}\right), \quad \text { and } \quad \Omega(Z)=\Psi^{\prime}+Z \psi_{0}+O\left(Z^{2}\right)
$$

onde $\beta \in \mathbb{R}\left(\beta=\Lambda^{\prime}(0)\right)$ e $\psi_{0} \in L^{2}\left(\psi_{0}=\Omega^{\prime}(0)\right)$. A afirmação do teorema seguirá se provarmos que $\beta<0$.

Dos Teoremas 3.6, 3.10, e do fato de $\Psi$ satisfazer a equação

$$
-\Psi^{\prime \prime}+\omega^{*} \Psi-\Psi^{3}=0
$$


obtemos que existe $\chi_{0} \in H^{1}$ para $Z$ perto de zero, tal que

$$
\Phi_{Z}=\Psi+Z \chi_{0}+O\left(Z^{2}\right) .
$$

Usando (4.29), e substituindo em (3.2), temos, para qualquer $\psi \in H^{1}$ do teorema de imersão de Sóbolev que

$$
Z\left\langle\mathcal{L}_{\Psi} \chi_{0}, \psi\right\rangle=Z[\Psi(c) \psi(c)+\Psi(-c) \psi(-c)]+O\left(Z^{2}\right)
$$

Assim derivando a expressão anterior em relação a $Z$, resulta

$$
\left\langle\mathcal{L}_{\Psi} \chi_{0}, \psi\right\rangle=\Psi(c) \psi(c)+\Psi(-c) \psi(-c)+O(Z) .
$$

Com o objetivo de determinar o sinal de $\beta$, a partir do sinal de $Z$; vamos desenvolver $\beta$ como função de $Z$. Para isso calcularemos $\left\langle\mathcal{L}_{1, Z} \Omega(Z), \Psi^{\prime}\right\rangle$ de duas formas distintas.

(1) Já que $\mathcal{L}_{1, Z} \Omega(Z)=\Lambda(Z) \Omega(Z)$, segue de (4.28) que

$$
\left\langle\mathcal{L}_{1, Z} \Omega(Z), \Psi^{\prime}\right\rangle=\beta Z\left\|\Psi^{\prime}\right\|^{2}+O\left(Z^{2}\right) .
$$

(2) Pela Proposição 4.4 temos,

$$
\begin{aligned}
\left\langle\mathcal{L}_{1, Z} \Omega(Z), \Psi^{\prime}\right\rangle & =t_{Z}\left[\Omega(Z), \Psi^{\prime}\right]=\left\langle(\Omega(Z))^{\prime}, \Psi^{\prime}\right\rangle-Z\left[\Omega(Z)(c) \Psi^{\prime}(c)+\Omega(Z)(-c) \Psi^{\prime}(-c)\right] \\
& +\omega\left\langle\Omega(Z), \Psi^{\prime}\right\rangle-\left\langle 3 \Phi_{Z}^{2} \Omega(Z), \Psi^{\prime}\right\rangle \\
& =-Z\left[\Omega(Z)(c) \Psi^{\prime}(c)+\Omega(Z)(-c) \Psi^{\prime}(-c)\right]+\left\langle\Omega(Z), \mathcal{L}_{\Psi} \Psi^{\prime}\right\rangle \\
& +\left(\omega-\omega^{*}\right)\left\langle\Omega(Z), \Psi^{\prime}\right\rangle-\left\langle 3\left[\Phi_{Z}^{2}-\Psi^{2}\right] \Omega(Z), \Psi^{\prime}\right\rangle .
\end{aligned}
$$

Estimaremos agora cada um dos dois últimos termos em (4.33).

- Como $\Phi_{Z}^{2}=\Psi^{2}+2 Z \Psi \chi_{0}+O\left(Z^{2}\right)$, segue de (4.28), de

$$
\mathcal{L}_{\Psi}\left(\omega^{*} \Psi-\Psi^{3}\right)=6 \Psi\left(\Psi^{\prime}\right)^{2}
$$

e da relação $\Phi^{2}(c)=\omega^{*}$ que

$$
\begin{aligned}
-\left\langle 3\left[\Phi_{Z}^{2}-\Psi^{2}\right] \Omega(Z), \Psi^{\prime}\right\rangle & =-Z\left\langle 6 \Psi \Psi^{\prime} \chi_{0}, \Psi^{\prime}\right\rangle+O\left(Z^{2}\right) \\
& =-Z\left\langle\mathcal{L}_{\Psi}\left(\omega^{*} \Psi-\Psi^{3}\right), \chi_{0}\right\rangle+O\left(Z^{2}\right) \\
& =-2 Z \Psi(c)\left[\omega^{*} \Psi(c)-\Psi^{3}(c)\right]+O\left(Z^{2}\right)=O\left(Z^{2}\right) .
\end{aligned}
$$

- Para $Z>0$ podemos mostrar que $\omega(k)-\omega^{*}=O(Z)$, para todo $k \in(0,1)$; e no caso $Z<0$ que $\omega(k)-\omega^{*}=O(Z)$, para $k \in\left(\frac{1}{\sqrt{2}}, 1\right)$ (ver Proposição 6.1 no Apêndice), desta maneira obtemos de (4.28) a relação

$$
\begin{aligned}
\left(\omega-\omega^{*}\right)\left\langle\Omega(Z), \Psi^{\prime}\right\rangle & =\left(\omega-\omega^{*}\right)\left\|\Psi^{\prime}\right\|^{2}+Z\left(\omega-\omega^{*}\right)\left\langle\psi_{0}, \Psi^{\prime}\right\rangle+O\left(Z^{2}\right) \\
& =\left(\omega-\omega^{*}\right)\left\|\Psi^{\prime}\right\|^{2}+O\left(Z^{2}\right) .
\end{aligned}
$$

- De (4.28) e da imersão de Sóbolev segue-se

$$
-Z\left[\Omega(Z)(c) \Psi^{\prime}(c)+\Omega(Z)(-c) \Psi^{\prime}(-c)\right]=-2 Z\left[\Psi^{\prime}(c)\right]^{2}+O\left(Z^{2}\right) .
$$


Logo, já que $\mathcal{L}_{\Psi} \Psi^{\prime}=0$, obtemos de (4.32)-(4.34)-(4.35)-(4.36) que

$$
\beta\left\|\Psi^{\prime}\right\|^{2}=\frac{\omega-\omega^{*}}{Z}\left\|\Psi^{\prime}\right\|^{2}-2\left[\Psi^{\prime}(c)\right]^{2}+O(Z) .
$$

Consideremos agora a função,

$$
S(k)=\frac{\omega(k)-\omega^{*}}{Z(k)}\left\|\Psi^{\prime}\right\|^{2}-2\left[\Psi^{\prime}(c)\right]^{2} .
$$

Então, dado que $Z \rightarrow 0$ se e somente se $k \rightarrow 1^{-}$podemos mostrar que

$$
\lim _{k \rightarrow 1^{-}} S(k)=L<0 .
$$

A constante $L$ depende do parâmetro $c$ (ver Proposição 6.1 no Apêndice). Finalmente, (4.37) e (4.39) permitem mostrar que $\beta<0$ para $|Z|$ pequeno (ver Corolário 6.2 no Apêndice). Finalmente, a primeira equação em (4.28) completa a prova.

(IV) Usando as informações anteriores e um argumento clássico baseado nas projetores de Riesz, poderemos deduzir o índice de Morse do operador $\mathcal{L}_{1, Z}$, para todo $Z$ admissível. Denotaremos o índice de Morse de $\mathcal{L}_{1, Z}$ por $n\left(\mathcal{L}_{1, Z}\right)$.

Teorema 4.10 Seja $Z \neq 0$ satisfazendo as condições nos Teoremas 3.6 e 3.10. Então

i. $\operatorname{para} Z>0, n\left(\mathcal{L}_{1, Z}\right)=2$,

ii. $\operatorname{para} Z<0, n\left(\mathcal{L}_{1, Z}\right)=1$.

Demonstração. Lembre que para $Z \neq 0, \operatorname{Ker}\left(\mathcal{L}_{1, Z}\right)=\{0\}$. Assim, considerando $Z<0$, definimos $Z_{\infty}$ por

$Z_{\infty}=\inf \left\{z<0: \mathcal{L}_{1, Z}\right.$ tem exatamente um autovalor negativo para todo $\left.Z \in(z, 0)\right\}$.

Segue-se do Teorema 4.9 que $Z_{\infty}$ está bem definido e $Z_{\infty} \in[-\infty, 0)$. Afirmamos que $Z_{\infty}=-\infty$. Suponha que $Z_{\infty}>-\infty$. Sejam $N=n\left(\mathcal{L}_{1, Z_{\infty}}\right)$ e $\Gamma$ uma curva fechada (por exemplo um círculo ou um retângulo) tal que

$$
0 \in \Gamma \subset \rho\left(\mathcal{L}_{1, Z_{\infty}}\right)
$$

e todos os autovalores negativos de $\mathcal{L}_{1, Z_{\infty}}$ pertencem ao domínio interno de $\Gamma$ (a região interior do plano que fica "dentro"da curva fechada $\Gamma$ ), então de (4.23) temos

$$
\mathcal{L}_{1, Z_{\infty}} \geqq M(c) I .
$$

Do Lema 4.5 segue que existe $\delta>0$ pequeno, tal que para $Z \in\left[Z_{\infty}-\delta, Z_{\infty}+\delta\right]$ temos $\Gamma \subset \rho\left(\mathcal{L}_{1, Z}\right)$, e para $\xi \in \Gamma$, a aplicação

$$
Z \rightarrow\left(\mathcal{L}_{1, Z}-\xi\right)^{-1}
$$

é analítica. Portanto, a existência de uma família analítica de projeções, $Z \rightarrow$ $P(Z)$, dada por

$$
P(Z)=-\frac{1}{2 \pi i} \int_{\Gamma}\left(\mathcal{L}_{1, Z}-\xi\right)^{-1} d \xi
$$


implica que,

$\operatorname{dim}(\operatorname{Rank} P(Z))=\operatorname{dim}\left(\operatorname{Rank} P\left(Z_{\infty}\right)\right)=N$, para todo $Z \in\left[Z_{\infty}-\delta, Z_{\infty}+\delta\right]$.

Segue-se então da definição de $Z_{\infty}$, que existe $z_{0} \in\left(Z_{\infty}, Z_{\infty}+\delta\right)$ tal que $\mathcal{L}_{1, Z}$ possui exatamente um autovalor negativo para todo $Z \in\left(z_{0}, 0\right)$. Assim, $\mathcal{L}_{1, Z_{\infty}+\delta}$ tem um autovalor negativo e $N=1$, donde $\mathcal{L}_{1, Z}$ tem um autovalor negativo para $Z \in\left(Z_{\infty}-\delta, 0\right)$; o que contradiz a definição de $Z_{\infty}$. Portanto, $Z_{\infty}=-\infty$. Uma análise similar pose ser aplicada para o caso $Z>0$.

Observação 4.11 A escolha da curva $\Gamma$ no começo da prova do Teorema 4.10, independe do parâmetro $-\infty<Z<0$. De fato, para toda $f \in \mathcal{D}$ com $\|f\|=1$, temos de (4.23)

$$
\left\langle\mathcal{L}_{1, Z} f, f\right\rangle \geqq-\gamma(c),
$$

with $\gamma(c)=\frac{4}{c^{2}}\left[d n^{-1}\left(\frac{1}{\sqrt{2}} ; \frac{1}{\sqrt{2}}\right)\right]^{2}$. Logo, inf $\sigma\left(\mathcal{L}_{1, Z}\right) \geqq-\gamma(c)$ para todo $-\infty<Z<$ 0 . Assim, $\Gamma$ pode ser escolhida como o retângulo $\Gamma=\partial R$, sendo

$$
R=\left\{z \in \mathbb{C}: z=z_{1}+i z_{2},\left(z_{1}, z_{2}\right) \in[-\gamma(c)-1,0] \times[-a, a] \text {, para algum } a>0\right\} .
$$

Proposição 4.12 A função $\Omega(Z)$ definida na Proposição 4.8, associada ao segundo autovalor negativo de $\mathcal{L}_{1, Z}$, pode ser extendida para $Z>0$ ao conjunto $I_{c}=\left(0, \frac{\pi}{4 c}\right)$, e para $Z<0$ ao conjunto $\mathcal{Q}_{c}=(-\infty, 0)$. Mais ainda, $\Omega(Z) \in H^{1}(\mathbb{R})$ é uma função impar de $Z$.

Demonstração. Temos, do Lema 4.5 e do Teorema XII.7 in [37] que o conjunto

$$
\Gamma_{0}=\left\{(Z, \lambda) \mid Z \in I_{c}, \lambda \in \rho\left(\mathcal{L}_{1, Z}\right)\right\}
$$

é aberto e $(Z, \lambda) \in \Gamma_{0} \rightarrow\left(\mathcal{L}_{1, Z}-\lambda\right)^{-1}$ é uma função analítica nas duas variáveis. Logo, podemos repetir o argumento dado na Proposição 4.8 em cada ponto $Z$, e em cada vizinhança de $Z$ para ver que as funções $\Omega(Z)$ e $\Pi(Z)$ são analíticas para $Z \in I_{c} \cup \mathcal{Q}_{c}$, ou seja, para cada $Z$ admissível. Consideremos o caso $Z<0$ (o caso $Z>0$ é similar). Sabemos da Proposição 4.3 e da Proposição 4.8, que a autofunção $\Omega(Z)$ é par ou ímpar e que $\Omega(0)=\frac{d}{d x} \Psi$ é ímpar. Então, da igualdade

$$
\lim _{Z \rightarrow 0}\langle\Omega(Z), \Omega(0)\rangle=\|\Omega(0)\|^{2} \neq 0
$$

temos $\langle\Omega(Z), \Omega(0)\rangle \neq 0$, para $Z$ perto de 0 . Logo, $\Omega(Z)$ é ímpar, em uma vizinhança do zero.

Seja $z_{\infty}$, definido por,

$$
z_{\infty}=\inf \{z<0: \Omega(Z) \text { é ímpar para cada } Z \in(z, 0]\} .
$$

Suponha que $z_{\infty}>-\infty$. Se $\Omega\left(z_{\infty}\right)$ é ímpar então, por continuidade, existe $\delta>0$ tal que $\Omega\left(z_{\infty}-\delta\right)$ é ímpar, o que é uma contradição. Logo, a Proposição 4.3 implica que $\Omega\left(z_{\infty}\right)$ é par. Por outro lado, já que $\Omega\left(z_{\infty}\right)$ é o limite de funções ímpares, obtemos que $\Omega\left(z_{\infty}\right)$ é ímpar. Portanto $\Omega\left(z_{\infty}\right) \equiv 0$, o que é impossível pois $\Omega\left(z_{\infty}\right)$ é uma autofunção. Assim, $z_{\infty}=-\infty$, e $\Omega(Z)$ é ímpar para todo $Z$ admissível. 


\subsection{Condição de Convexidade}

Dedicamos esta seção ao estudo da slope condition, requerida para o nosso resultado de estabilidade/instabilidade. De maneira precisa, provaremos que se $\omega$ é admissível, i.e., $\omega \in I_{c} \cup P_{c}$, sendo $I_{c}$ e $P_{c}$ como definidos nas observações 3.5 e 3.9, respectivamente; a aplicação $\omega \mapsto\left\|\Phi_{\omega}\right\|_{2}^{2}$ tem derivada positiva. Desta maneira concluiremos o resultado de estabilidade/instabilidade para a órbita $\Omega_{\Phi_{\omega}}=\left\{e^{i \theta} \Phi_{\omega}: \theta \in[0,2 \pi]\right\}$ estabelecido no Teorema 0.2. Como o cálculo da derivada da norma $L^{2}$ do profile $\Phi_{\omega}$ torna-se altamente complicada pelo uso de específicas integrais elípticas, foi necessário o uso de ferramentas de tipo computacional para finalizar nosso estudo de estabilidade.

Teorema 4.13 Seja $\omega>0$ como nos Teoremas 3.6 ou 3.10. Assim, para $Z \in$ $\left(0, \frac{\pi}{4 c}\right)$ temos $\omega \in I_{c}=\left(\omega^{*}, \frac{\pi^{2}}{8 c^{2}}\right)$, e para $Z \in(-\infty, 0)$ temos $\omega \in\left(0, \omega^{*}\right)$; onde

$$
\omega^{*}=\left(\frac{\operatorname{sech}^{-1}\left(\frac{1}{\sqrt{2}}\right)}{c}\right)^{2}
$$

Então $\partial_{\omega}\left\|\Phi_{\omega}\right\|_{2}^{2}>0$, para $\Phi_{\omega}$ representando qualquer um dos perfis de tipo dnoidal ou cnoidal, dados nos Teoremas 3.6 e 3.10, respectivamente.

Demonstração. Dividiremos a prova nos casos $Z>0$ e $Z<0$.

1) Caso $Z>0$, perfil dnoidal. Das relações dadas em (3.7) e (3.29) podemos escrever os parâmetros $\beta=\sqrt{\frac{\omega}{2-k^{2}}}$ e $\alpha=\sqrt{2} \beta$ como funções do módulo $k \in$ $(0,1)$, assim:

$$
\beta(k)=\frac{1}{c} c n^{-1}\left(\frac{1}{\sqrt{2}} ; k\right) .
$$

Portanto, o perfil dnoidal $\Phi_{\omega}$ pode ser visto como uma função suave de $k$,

$$
\phi_{k}(x)=\sqrt{2} \beta(k) d n(x \beta(k) ; k), \quad \text { para } x \in[-c, c],
$$

e

$$
\varphi_{b(k)}(x)=\sqrt{2 \omega(k)} \operatorname{sech}\left(\sqrt{\omega(k)}(x-c)+\operatorname{sech}^{-1}\left(\frac{1}{\sqrt{2}}\right)\right),
$$

para $x \in[c,+\infty)$. Para $x \leq-c, \Phi_{\omega}(x)=\varphi_{b(k)}(-x)$.

Agora, para $N(\omega)=\left\|\Phi_{\omega}\right\|_{2}^{2}$, consideramos a função suave $k \mapsto N(\omega(k))$. A regra da cadeia implica

$$
\frac{d}{d k}\left(N(\omega(k))=N^{\prime}(\omega) \frac{d}{d k} \omega(k) .\right.
$$

Logo, já que para todo $k \in(0,1)$ temos (ver figura 3.6)

$$
\frac{d}{d k} \omega(k)=\frac{1}{c^{2}}\left[-2 k\left(c n^{-1}\left(\frac{1}{\sqrt{2}} ; k\right)\right)^{2}+2\left(2-k^{2}\right) c n^{-1}\left(\frac{1}{\sqrt{2}} ; k\right) \frac{\partial}{\partial_{k}} c n^{-1}\left(\frac{1}{\sqrt{2}} ; k\right)\right]<0,
$$

seguirá, de (4.40), que se $\frac{d}{d k} N(\omega(k))<0$ então $\frac{d}{d \omega} N(\omega)>0$. Da paridade de $\Phi_{\omega}$ segue-se

$$
N(\omega(k))=2\left[\int_{0}^{c} \phi_{k}^{2}(x) d x+\int_{c}^{+\infty} \varphi_{b(k)}^{2}(x) d x\right] .
$$


Para $c \leqq x$ definamos $a_{k}(x) \equiv \sqrt{\omega(k)}(x-c)+\operatorname{sech}^{-1}\left(\frac{1}{\sqrt{2}}\right)$, logo pela construção do perfil dnoidal, $\tanh \left(a_{k}(c)\right)=\frac{1}{\sqrt{2}}$. Temos então

$$
\begin{aligned}
2 \int_{c}^{\infty} \varphi_{b(k)}^{2}(x) d x & =4 \frac{\left(2-k^{2}\right)\left(c n^{-1}\left(\frac{1}{\sqrt{2}} ; k\right)\right)^{2}}{c^{2}} \int_{c}^{\infty} \operatorname{sech}^{2}\left(a_{k}(x)\right) d x \\
& =4 \frac{\left(2-k^{2}\right)\left(c n^{-1}\left(\frac{1}{\sqrt{2}} ; k\right)\right)^{2}}{c^{2}}\left[\left.\frac{1}{\sqrt{\omega(k)}} \tanh \left(a_{k}(x)\right)\right|_{c} ^{\infty}\right] \\
& =\frac{4}{c} \sqrt{2-k^{2}} c n^{-1}\left(\frac{1}{\sqrt{2}} ; k\right)\left(\frac{\sqrt{2}-1}{\sqrt{2}}\right) .
\end{aligned}
$$

Por outro lado,

$$
2 \int_{0}^{c} \phi_{k}^{2}(x) d x=4 \frac{\left(c n^{-1}\left(\frac{1}{\sqrt{2}} ; k\right)\right)^{2}}{c^{2}} \int_{0}^{c} d n^{2}(x \beta(k)) d x .
$$

Fazendo a mudança de variável $x \beta(k) \equiv u$, obtemos

$$
\begin{aligned}
2 \int_{0}^{c} \phi_{k}^{2}(x) d x & =4 \frac{c n^{-1}\left(\frac{1}{\sqrt{2}} ; k\right)}{c} \int_{0}^{c \beta(k)} d n^{2}(u) d u \\
& =4 \frac{c n^{-1}\left(\frac{1}{\sqrt{2}} ; k\right)}{c} E\left(\frac{\pi}{4} ; k\right) .
\end{aligned}
$$

Note que $c \beta(k)=c n^{-1}\left(\frac{1}{\sqrt{2}} ; k\right)$. Aqui $E(\cdot ; k)$ representa a integral incompleta de segundo tipo, comentada no começo da tese. Segue-se então que

$$
N(\omega(k))=\frac{4}{c} c n^{-1}\left(\frac{1}{\sqrt{2}} ; k\right)\left[\sqrt{2-k^{2}}\left(\frac{\sqrt{2}-1}{\sqrt{2}}\right)+E\left(\frac{\pi}{4} ; k\right)\right]
$$

A seguir, notamos que o sinal da derivada de $N(\omega(k))$ independe do ponto de interação c. A figura 4.1 mostra o gráfico da função $D(k)=\frac{c}{4} N(\omega(k))$, para $k \in(0,1)$. Vemos, apartir deste gráfico que $\frac{d}{d k} N(\omega(k))<0$; portanto $\frac{d}{d \omega} N(\omega)>0$.

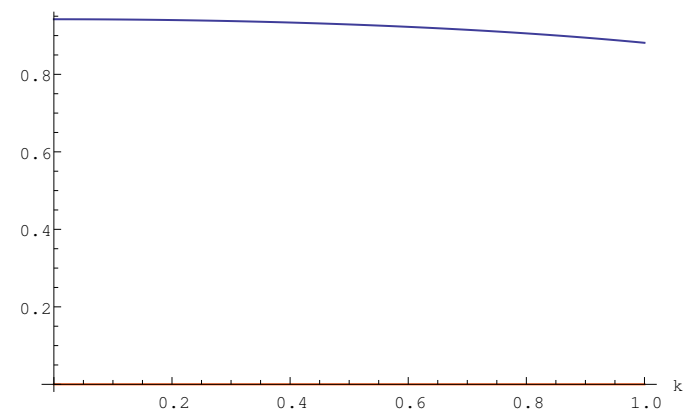

Figura 4.1: Gráfico da função $D(k)$ 
2) Caso $Z<0$, perfil cnoidal. A partir do Teorema 3.10 podemos escrever os parâmetros $\omega, \tilde{\beta}=\sqrt{\frac{\omega}{2 k^{2}-1}}$ e $\tilde{\alpha}=\sqrt{\frac{\omega}{2 k^{2}-1}} k$ como funções do módulo $k \in\left(\frac{1}{\sqrt{2}}, 1\right)$. A saber,

$$
\tilde{\beta}(k)=\frac{1}{c} d n^{-1}\left(\frac{1}{\sqrt{2}} ; k\right)
$$

e

$$
\omega(k)=\omega_{k}=\frac{2 k^{2}-1}{c^{2}}\left(d n^{-1}\left(\frac{1}{\sqrt{2}} ; k\right)\right)^{2}
$$

Logo, o perfil cnoidal no Teorema 3.10 está dado por

$$
\tilde{\phi}_{k}(x)=\sqrt{2} k \tilde{\beta}(k) c n(x \tilde{\beta}(k) ; k), \text { para } x \in[-c, c]
$$

e

$$
\tilde{\varphi}_{d(k)}(x)=\sqrt{2 \omega(k)} \operatorname{sech}\left(\sqrt{\omega(k)}(x-c)+\operatorname{sech}^{-1}\left(\frac{1}{\sqrt{2}}\right)\right), \text { para } x \in[c, \infty) .
$$

Para $x \leq-c, \Phi_{\omega}(x)=\tilde{\varphi}_{d(k)}(-x)$.

Da mesma maneira que no caso dnoidal $Z>0$, para $N(\omega)=\left\|\Phi_{\omega}\right\|_{2}^{2}$, consideramos a função suave $k \mapsto N(\omega(k))$. A regra da cadeia implica então

$$
\frac{d}{d k}(N(\omega(k)))=N^{\prime}(\omega) \frac{d}{d k} \omega(k) .
$$

Dado que para $k \in\left(\frac{1}{\sqrt{2}}, 1\right)$ (ver gráfica 3.10$)$ temos

$$
\frac{d}{d k} \omega(k)=\frac{1}{c^{2}}\left[4 k\left(d n^{-1}\left(\frac{1}{\sqrt{2}} ; k\right)\right)^{2}+2\left(2 k^{2}-1\right) d n^{-1}\left(\frac{1}{\sqrt{2}} ; k\right) \frac{\partial}{\partial k} d n^{-1}\left(\frac{1}{\sqrt{2}} ; k\right)\right]>0,
$$

seguirá, de (4.41) que se $\frac{d}{d k} N(\omega(k))>0$ então $\frac{d}{d \omega} N(\omega)>0$. Um cálculo totalmente análogo ao caso $Z>0$ mostra que, para $v_{k}(x)=\sqrt{\omega(k)}(x-c)+$ $\operatorname{sech}^{-1}\left(\frac{1}{\sqrt{2}}\right)(c \leqq x)$ temos

$$
\begin{aligned}
2 \int_{0}^{\infty} \tilde{\varphi}_{d(k)}^{2}(x) d x & =\frac{\left(2 k^{2}-1\right)\left(d n^{-1}\left(\frac{1}{\sqrt{2}} ; k\right)\right)^{2}}{c^{2}} \int_{c}^{\infty} \operatorname{sech}^{2}\left(v_{k}(x)\right) d x \\
& =\frac{4}{c} \sqrt{2 k^{2}-1} d n^{-1}\left(\frac{1}{\sqrt{2}} ; k\right)\left(\frac{\sqrt{2}-1}{\sqrt{2}}\right)
\end{aligned}
$$

e

$$
\begin{aligned}
2 \int_{0}^{c} \tilde{\phi}_{k}^{2}(x) d x & =\frac{4}{c^{2}}\left(d n^{-1}\left(\frac{1}{\sqrt{2}} ; k\right)\right)^{2} \int_{0}^{c} k^{2} c n^{2}(x \tilde{\beta}(k)) d x \\
& =\frac{4}{c^{2}}\left(d n^{-1}\left(\frac{1}{\sqrt{2}} ; k\right)\right)^{2}\left[c\left(k^{2}-1\right)+\int_{0}^{c} d n^{2}(x \tilde{\beta}(k)) d x\right] \\
& =\frac{4}{c} d n^{-1}\left(\frac{1}{\sqrt{2}} ; k\right)\left[\left(k^{2}-1\right) d n^{-1}\left(\frac{1}{\sqrt{2}} ; k\right)+E\left(\operatorname{sen}^{-1}\left(\frac{1}{k \sqrt{2}}\right) ; k\right)\right] .
\end{aligned}
$$


Aqui, $\operatorname{sen}^{-1}(\cdot)$ é a função seno inverso. A quantidade $\operatorname{sen}^{-1}\left(\frac{1}{\sqrt{2} k}\right)$ está bem definida, por quanto $\frac{1}{2}<k^{2}$. Portanto,

$$
\begin{aligned}
N(\omega(k))= & 2 \int_{0}^{\infty} \tilde{\varphi}_{d(k)}^{2}(x) d x+2 \int_{0}^{c} \tilde{\phi}_{k}^{2}(x) d x= \\
& \frac{4}{c} \sqrt{2 k^{2}-1} d n^{-1}\left(\frac{1}{\sqrt{2}} ; k\right)\left(\frac{\sqrt{2}-1}{\sqrt{2}}\right) \\
+ & \frac{4}{c} d n^{-1}\left(\frac{1}{\sqrt{2}} ; k\right)\left[\left(k^{2}-1\right) d n^{-1}\left(\frac{1}{\sqrt{2}} ; k\right)+E\left(\operatorname{sen}^{-1}\left(\frac{1}{k \sqrt{2}}\right) ; k\right)\right] .
\end{aligned}
$$

De novo, note que o ponto $c$ não intervêm no cáculo da derivada. A figura 4.4 abaixo mostra o gráfico da função $C(k)=\frac{c}{4} N^{\prime}(\omega(k))$. Concluímos deste gráfico

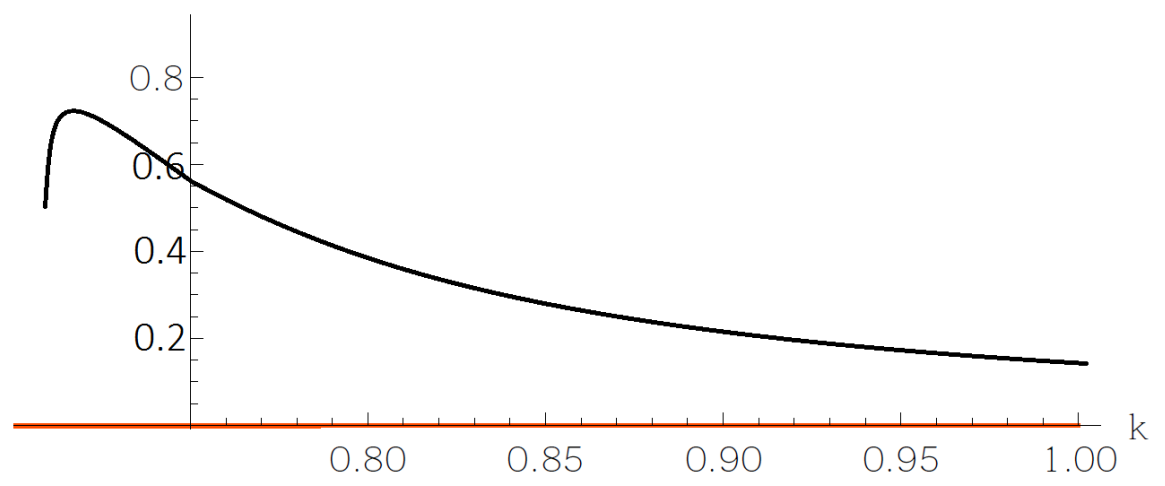

Figura 4.2: Gráfico da função $C(k)$

que $\frac{d}{d k} N(\omega(k))>0$, e portanto $\frac{d}{d \omega} N(\omega)>0$. Isto finalize a prova do Teorema

\subsection{Prova do Teorema 0.2}

A partir das últimas seções já será possível estabelecer a prova do nosso resultado de estabilidade/instabilidade, Teorema 0.2, associado à órbita $\Omega_{\Phi_{\omega, Z, c}}=\left\{e^{i \theta} \Phi_{\omega, Z, c}\right.$ : $\theta \in[0,2 \pi]\}$ gerada pelas soluções peak-standing waves $\Phi_{\omega, Z, c}$ como em (3.33) no Teorema 3.6, para o caso $Z>0$; ou então $\tilde{\Phi}_{\omega, Z, c}$ como em (3.45) no Teorema 3.10, para $Z<0$. O resultado seguirá como consequência do Teorema 1.2 de Grillakis et. al e estabelecido no Capítulo 1. A prova é como segue.

Demonstração. Sabemos, pela construção, que os perfis $\Phi_{\omega, Z, c}$ e $\tilde{\Phi}_{\omega, Z, c}$ estão associados à solução standing-wave do problema (7). A existência global para o (PVI) (7) foi estabelecida na Proposição 2.3 do capítulo 2. Os Lemas 4.1 e 4.2 garantem que $\operatorname{Ker}\left(\mathcal{L}_{2, Z, c}\right)=\left[\Phi_{\omega, Z, c}\right]$ e $\operatorname{Ker}\left(\mathcal{L}_{1, Z, c}\right)=\{0\}$, respectivamente. Agora, pelo Lema 4.13 segue que $p(\omega)=1$ no Teorema 1.2 .

Por outro lado, o Teorema 4.10 garante que, para $Z>0$ o operador $\mathcal{L}_{1, Z, c}$ possui exatamente dois autovalores negativos. Já para $Z<0$ o Teorema 4.10 garante que $\mathcal{L}_{1, Z, c}$ possui apenas um autovalor negativo. Assim, para $Z>0$ temos

$$
n\left(\mathcal{H}_{\omega, Z, c}\right)-p(\omega)=1
$$


e para $Z<0$,

$$
n\left(\mathcal{H}_{\omega, Z, c}\right)=p(\omega)=1 .
$$

Portanto, a prova dos items 1. e 2. do Teorema 0.2 segue-se aplicando o Teorema 1.2 .

Agora, Proposição 4.12 assegura que o segundo autovalor de $\mathcal{L}_{1, Z, c}$ está associado com uma autofunção ímpar, e assim este irá a desaparecer quando o problema seja restrito ao subespaço das funções pares. Além disso, dado que $\Phi_{\omega, Z, c}$ é uma função par e trivialmente satisfaz que

$$
\left\langle\mathcal{L}_{1, Z, c} \Phi_{\omega, Z, c}, \Phi_{\omega, Z, c}\right\rangle<0
$$

para $Z>0$, obtemos que o primeiro autovalor negativo de $\mathcal{L}_{1, Z, c}$ ainda está presente quando o problema é restrito ao subespaço das funções pares de $H^{1}(\mathbb{R})$, ou seja, $H_{\text {par }}^{1}(\mathbb{R})$. Portanto, obtemos neste caso que

$$
n\left(\left.\mathcal{H}_{\omega, Z, c}\right|_{H_{p a r}^{1}}\right)=1 \text {. }
$$

Portanto, o item 3. do Teorema 0.2 resulta do item 1 . do Teorema 1.2 e do Teorema 2.3. Isso termina a prova do teorema. 


\section{Capítulo 5}

\section{Estudos futuros}

A generalização natural que admite o modelo NLS- $\delta_{ \pm c}(7)$ é a seguinte: considere a equação

$$
\left\{\begin{array}{l}
i \partial_{t} u(x, t)+\partial_{x}^{2} u(x, t)+\left[Z_{1} \delta_{a}(x)+Z_{2} \delta_{b}(x)\right] u(x, t)=-|u(x, t)|^{2} u(x, t), \\
u(x, 0)=u_{0}(x)
\end{array}\right.
$$

sendo $u: \mathbb{R} \times \mathbb{R} \longrightarrow \mathbb{C}, Z_{1}, Z_{2}, a, b \in \mathbb{R} ;$ e $\delta_{a}$ e $\delta_{b}$ as distribuições de Dirac agindo nos pontos $x=a$ e $x=b$; respectivamente. Surgem daqui dois problemas naturais: o primeiro é o de determinar, se possível, soluções do tipo standing-wave associadas ao modelo (5.1); e o segundo, no caso de existirem tais soluções, decidir se elas são orbitalmente estáveis/instáveis no espaço $H^{1}(\mathbb{R})$.

É claro que a equação (5.1) fornece, a partir da escolha dos parâmetros $a, b, Z_{1}$ e $Z_{2}$, equações particulares com dupla interação de Dirac. Por exemplo, assumamos que os sinais de $Z_{1}$ e $Z_{2}$ são os mesmos, consideremos o seguinte modelo:

Para $a$ e $b$ não nulos, com $a=-b$ e $Z_{1}<Z_{2}$

$$
\left\{\begin{array}{l}
i \partial_{t} u(x, t)+\partial_{x}^{2} u(x, t)+\left[Z_{1} \delta_{a}(x)+Z_{2} \delta_{-a}(x)\right] u(x, t)=-|u(x, t)|^{2} u(x, t), \\
u(x, 0)=u_{0}(x) .
\end{array}\right.
$$

Seja $Z \equiv\left\{Z_{1}, Z_{2}\right\}$. Então, se $u(x, t)=e^{i \omega t} \zeta_{\omega, Z, a}$ é uma solução standing-wave associada à equação $(5.2), \zeta=\zeta_{\omega, Z, c}$ deve satisfazer, no sentido das distribuições, a equação

$$
-\zeta^{\prime \prime}+\omega \zeta-\zeta^{3}-Z_{1} \delta_{a} \zeta-Z_{2} \delta_{-a} \zeta \equiv 0 .
$$

Mais precisamente, para $x \in \mathbb{R}-\{a,-a\}, \zeta$ satisfaz

$$
-\zeta^{\prime \prime}(x)+\omega \zeta(x)-\zeta^{3}(x)=0,
$$

e a condição de fronteira

$$
\left\{\begin{array}{l}
\zeta^{\prime}\left(a^{+}\right)-\zeta^{\prime}\left(a^{-}\right)=-Z_{1} \zeta(a) \\
\zeta^{\prime}\left(-a^{+}\right)-\zeta^{\prime}\left(-a^{-}\right)=-Z_{2} \zeta(-a)
\end{array}\right.
$$

Para este tipo particular de modelo (5.2) -que de fato constitue uma leve extensão de (7), é possível construir um perfil $\zeta$ seguindo as mesmas ideias no caso em que $Z_{1}=Z_{2}$, isto é: para $c<|x|$ consideramos uma translação do soliton-perfil secante 
hiperbólica (3.4); e para $|x| \leqq c$ consideramos perfis periódicos do tipo dnoidal e cnoidal. De fato, podem-se seguir as mesmas técnicas que no caso tratado nesta tese -em particular o que tem a ver com a localização dos pontos de interção $a$ e - a; como mostrado na Proposição 3.4. O problema mais difícil de tratar para este tipo de modelos radica no cálculo do índice de Morse para o operador associado

$$
\mathcal{L}_{1, Z}=-\frac{d^{2}}{d x^{2}}+\omega-3 \zeta^{2}
$$

com domínio

$D\left(\mathcal{L}_{1, Z}\right)=\left\{f \in \mathcal{D}: f^{\prime}\left(a^{+}\right)-f^{\prime}\left(a^{-}\right)=-Z_{1} f(a) ; f^{\prime}\left(-a^{+}\right)-f^{\prime}\left(-a^{-}\right)=-Z_{2} f(-a)\right\}$,

onde $\mathcal{D}=H^{1}(\mathbb{R}) \cap H^{2}(\mathbb{R}-\{ \pm a\})$.

Haver suposto que $Z_{1}$ e $Z_{2}$ possuem o mesmo sinal e que $Z_{1}<Z_{2}$ garantem que, no caso em que $Z_{1}$ e $Z_{2}$ sejam negativos; tenhamos

$$
Z_{1} \rightarrow 0^{-} \Longrightarrow Z_{2} \rightarrow 0^{-}
$$

e para o caso em que $Z_{1}$ e $Z_{2}$ sejam positivos

$$
Z_{2} \rightarrow 0^{+} \Longrightarrow Z_{1} \rightarrow 0^{+} \text {. }
$$

Estes dois fatos são muito importantes para as convergências dos respectivos perfis dnoidal e cnoidal ajustados entre os pontos $a$ e $-a$; para uma função do tipo secante hiperbólica, semelhante aos resultados obtidos nos Teoremas 3.6 e 3.10. Já sobre isso temos um trabalho em andamento.

Um outro caso particular do modelo (5.1) é supor que $a$ e $b$ não possuem nenhuma relação a priori, do tipo $a=-b$. Neste caso, para a correspondente equação diferencial resultante, fica complexo determinar um perfil associado à soluções standing-wave -porquanto perdemos a vital condição de paridade (ganha no caso especial em que $a=-b$ ). 


\section{Capítulo 6}

\section{Apêndice}

Justificaremos a seguir várias afirmações que usamos ao longo do nosso trabalho. A seguinte proposição foi usada para provar o Teorem 4.9.

Proposição 6.1 Considere o soliton $\Psi(x)=\sqrt{2 \omega^{*}} \operatorname{sech}\left(\sqrt{\omega^{*}} x\right)$, com $\omega^{*}=\frac{1}{c^{2}}\left[\operatorname{sech}^{-1}\left(\frac{1}{\sqrt{2}}\right)\right]^{2}$. Para $Z=Z(k)>0$ e $\omega=\omega(k)$ como definidos em (3.28); e para $Z=Z(k)<0$ e $\omega=\omega(k)$ como definidos em (3.41), consideremos a função

$$
S(k)=\frac{\omega(k)-\omega^{*}}{Z(k)}\left\|\Psi^{\prime}\right\|^{2}-2\left[\Psi^{\prime}(c)\right]^{2} .
$$

Então,

$$
\lim _{k \rightarrow 1^{-}} S(k)=\frac{\left[\operatorname{sech}^{-1}\left(\frac{1}{\sqrt{2}}\right)\right]^{2}}{c^{4}}\left[\frac{4 l}{3 \sqrt{2}}-\left[\operatorname{sech}^{-}\left(\frac{1}{\sqrt{2}}\right)\right]^{2}\right] \equiv L<0,
$$

com $l=\frac{1}{2} \ln (1+\sqrt{2})[-\sqrt{2}+3 \ln (1+\sqrt{2})] \approx 0.5420038597$. Mais ainda, para $Z>0$ temos $\omega(k)-\omega^{*}>0$, e para $Z<0$ temos $\omega(k)-\omega^{*}<0$, com $\omega(k)-\omega^{*}=O(Z)$.

Demonstração. Consideremos primeiro o caso $Z>0$ (perfil dnoidal como no Teorema (3.6)). Então,

$$
Z=Z(k)=\frac{\sqrt{2}}{c} \frac{1-k^{2}}{\sqrt{2-k^{2}}} c n^{-1}\left(\frac{1}{\sqrt{2}} ; k\right),
$$

$\mathrm{e}$

$$
\omega(k)=\frac{2-k^{2}}{c^{2}}\left[c n^{-1}\left(\frac{1}{\sqrt{2}} ; k\right)\right]^{2} .
$$

Já que para todo $k \in(0,1)$ temos

$$
\frac{\pi}{4}<\operatorname{cn}^{-1}\left(\frac{1}{\sqrt{2}} ; k\right)<\operatorname{sech}^{-1}\left(\frac{1}{\sqrt{2}}\right)<1,
$$

segue que

$$
\begin{aligned}
\left|\omega(k)-\omega^{*}\right| & =\frac{1}{c^{2}}\left|\left(2-k^{2}\right)\left[\operatorname{cn}^{-1}\left(\frac{1}{\sqrt{2}} ; k\right)\right]^{2}-\left[\operatorname{sech}^{-1}\left(\frac{1}{\sqrt{2}}\right)\right]^{2}\right| \\
& =\frac{1}{c^{2}}\left|\left(1-k^{2}\right)\left[\operatorname{cn}^{-1}\left(\frac{1}{\sqrt{2}} ; k\right)\right]^{2}+\left[\operatorname{cn}^{-1}\left(\frac{1}{\sqrt{2}} ; k\right)\right]^{2}-\left[\operatorname{sech}^{-1}\left(\frac{1}{\sqrt{2}}\right)\right]^{2}\right| \\
& \leq \frac{1-k^{2}}{c^{2}}\left[c n^{-1}\left(\frac{1}{\sqrt{2}} ; k\right)\right]^{2}+\frac{1}{c^{2}}\left|\left[\operatorname{cn}^{-1}\left(\frac{1}{\sqrt{2}} ; k\right)\right]^{2}-\left[\operatorname{sech}^{-1}\left(\frac{1}{\sqrt{2}}\right)\right]^{2}\right| .
\end{aligned}
$$


De (6.1) segue

$$
\frac{1-k^{2}}{c^{2}}\left[c n^{-1}\left(\frac{1}{\sqrt{2}} ; k\right)\right]^{2} \leq \frac{1-k^{2}}{c^{2}} c n^{-1}\left(\frac{1}{\sqrt{2}} ; k\right)=Z \frac{\sqrt{2-k^{2}}}{c \sqrt{2}} \leq \frac{Z}{c} .
$$

Por outro lado, também de (6.1) segue

$$
\frac{1}{c^{2}}\left|\left[\operatorname{cn}^{-1}\left(\frac{1}{\sqrt{2}} ; k\right)\right]^{2}-\left[\operatorname{sech}^{-1}\left(\frac{1}{\sqrt{2}}\right)\right]^{2}\right| \leq \frac{2 \operatorname{sech}^{-1}\left(\frac{1}{\sqrt{2}}\right)}{c^{2}}\left(\operatorname{sech}^{-1}\left(\frac{1}{\sqrt{2}}\right)-\operatorname{cn}^{-1}\left(\frac{1}{\sqrt{2}} ; k\right) .\right)
$$

Além disso, é possível mostrar que para todo $k \in(0,1)$ obtemos

$$
0<\operatorname{sech}^{-1}\left(\frac{1}{\sqrt{2}}\right)-\operatorname{cn}^{-1}\left(\frac{1}{\sqrt{2}} ; k\right)<1-k^{2} .
$$

Portanto, (6.1) implica

$$
\operatorname{sech}^{-1}\left(\frac{1}{\sqrt{2}}\right)-c n^{-1}\left(\frac{1}{\sqrt{2}} ; k\right)<1-k^{2}=Z \frac{c}{\sqrt{2}} \frac{\sqrt{2-k^{2}}}{c n^{-1}\left(\frac{1}{\sqrt{2}} ; k\right)}<Z \frac{c}{c n^{-1}\left(\frac{1}{\sqrt{2}} ; k\right)}<Z \frac{4 c}{\pi} .
$$

Finalmente, da desigualdade (6.3) obtemos

$$
\frac{1}{c^{2}}\left|\left[\operatorname{cn}^{-1}\left(\frac{1}{\sqrt{2}} ; k\right)\right]^{2}-\left[\operatorname{sech}^{-1}\left(\frac{1}{\sqrt{2}}\right)\right]^{2}\right| \leq Z \frac{8}{c \pi} \operatorname{sech}^{-1}\left(\frac{1}{\sqrt{2}}\right) .
$$

Desta maneira, das desigualdade (6.2) e (6.4) temos, para todo $k \in(0,1)$,

$$
\left|\omega(k)-\omega^{*}\right| \leq\left(\frac{1}{c}+\frac{8 \operatorname{sech}^{-1}\left(\frac{1}{\sqrt{2}}\right)}{c \pi}\right) Z .
$$

Portanto, $\omega(k)-\omega^{*}=O(Z)$. Além do mais, é fácil obter que $\omega^{*}<\omega(k)$ para todo $k \in(0,1)$.

Um cálculo simples mostra que

$$
\left\|\Psi^{\prime}\right\|^{2}=\left.\frac{2}{3} \frac{\left[\operatorname{sech}^{-1}\left(\frac{1}{\sqrt{2}}\right)\right]^{3}}{c^{3}} \tanh ^{3}\left(\sqrt{\omega^{*}} x\right)\right|_{-\infty} ^{\infty}=\frac{4}{3 c^{3}}\left[\operatorname{sech}^{-1}\left(\frac{1}{\sqrt{2}}\right)\right]^{3},
$$

e do valor específico de $\omega^{*}$ segue que $\operatorname{sech}^{2}\left(\sqrt{\omega^{*}} c\right)=\tanh ^{2}\left(\sqrt{\omega^{*}} c\right)=\frac{1}{2}$. Portanto,

$$
\left[\Psi^{\prime}(c)\right]^{2}=\frac{1}{2}\left(\omega^{*}\right)^{2}=\frac{1}{2 c^{4}}\left[\operatorname{sech}^{-1}\left(\frac{1}{\sqrt{2}}\right)\right]^{4} .
$$

Usando o programa WolframMathematica determinamos exatamente a quantidade

$$
\lim _{k \rightarrow 1^{-}} \frac{1}{1-k^{2}}\left[\left(2-k^{2}\right)\left(c n^{-1}\left(\frac{1}{\sqrt{2}} ; k\right)\right)^{2}-\left(\operatorname{sech}^{-1}\left(\frac{1}{\sqrt{2}}\right)\right)^{2}\right]=l,
$$

sendo que $l=\frac{1}{2} \ln (1+\sqrt{2})[-\sqrt{2}+3 \ln (1+\sqrt{2})] \approx 0.5420038597$. Assim, segue de (6.5) e da continuidade das funções $k \longmapsto c n^{-1}\left(\frac{1}{\sqrt{2}} ; k\right)$ e $k \longmapsto \sqrt{2-k^{2}}$ que

$$
\lim _{k \rightarrow 1^{-}} \frac{\omega(k)-\omega^{*}}{Z(k)}=\frac{l}{c \sqrt{2} \operatorname{sech}^{-1}\left(\frac{1}{\sqrt{2}}\right)}>0
$$


A análise acima mostra que

$$
\lim _{k \rightarrow 1^{-}} \frac{\omega(k)-\omega^{*}}{Z(k)}\left\|\Psi^{\prime}\right\|^{2}-2\left[\Psi^{\prime}(c)\right]^{2}=\frac{\left(\operatorname{sech}^{-1}\left(\frac{1}{\sqrt{2}}\right)\right)^{2}}{c^{4}}\left[\frac{4 l}{3 \sqrt{2}}-\left(\operatorname{sech}^{-1}\left(\frac{1}{\sqrt{2}}\right)\right)^{2}\right]<0 .
$$

Consideremos agora o caso $Z<0$ (perfil cnoidal como no Teorema 3.10). Neste caso, para $k \in\left(\frac{1}{\sqrt{2}}, 1\right)$ obtemos:

$$
Z=Z(k)=-\frac{\sqrt{2}}{c} \frac{1-k^{2}}{\sqrt{2 k^{2}-1}} d n^{-1}\left(\frac{1}{\sqrt{2}} ; k\right)
$$

e

$$
\omega(k)=\frac{2 k^{2}-1}{c^{2}}\left[d n^{-1}\left(\frac{1}{\sqrt{2}} ; k\right)\right]^{2} .
$$

Já que $k \in\left(\frac{1}{\sqrt{2}}, 1\right)$ então

$$
\left[\operatorname{sech}^{-1}\left(\frac{1}{\sqrt{2}}\right)\right]^{2}-k^{2}\left[d n^{-1}\left(\frac{1}{\sqrt{2}} ; k\right)\right]^{2}<0 .
$$

Agora,

$$
\begin{aligned}
\left|\omega(k)-\omega^{*}\right| & =\frac{1}{c^{2}}\left|\left(2 k^{2}-1\right)\left(d n^{-1}\left(\frac{1}{\sqrt{2}} ; k\right)\right)^{2}-\left(\operatorname{sech}^{-1}\left(\frac{1}{\sqrt{2}}\right)\right)^{2}\right| \\
& =\frac{1}{c^{2}}\left[\left(\operatorname{sech}^{-1}\left(\frac{1}{\sqrt{2}}\right)\right)^{2}-\left(2 k^{2}-1\right)\left(d n^{-1}\left(\frac{1}{\sqrt{2}} ; k\right)\right)^{2}\right] \\
& \leq \frac{1-k^{2}}{c^{2}}\left(d n^{-1}\left(\frac{1}{\sqrt{2}} ; k\right)\right)^{2} .
\end{aligned}
$$

Mas,

$$
\frac{1-k^{2}}{c^{2}}\left(d n^{-1}\left(\frac{1}{\sqrt{2}} ; k\right)\right)^{2}=|Z| \frac{\sqrt{2 k^{2}-1}}{c \sqrt{2}} d n^{-1}\left(\frac{1}{\sqrt{2}} ; k\right) \leq|Z| \frac{\operatorname{sech}^{-1}\left(\frac{1}{\sqrt{2}}\right)}{c \sqrt{2}} .
$$

Obtemos da última desigualdade que

$$
\left|\omega(k)-\omega^{*}\right| \leq|Z| \frac{\operatorname{sech}^{-1}\left(\frac{1}{\sqrt{2}}\right)}{c \sqrt{2}} .
$$

Usando de novo o programa WolframMathematica determinamos o seguinte limite

$$
\lim _{k \rightarrow 1^{-}} \frac{1}{1-k^{2}}\left[\left(2 k^{2}-1\right)\left(d n^{-1}\left(\frac{1}{\sqrt{2}} ; k\right)\right)^{2}-\left(\operatorname{sech}^{-1}\left(\frac{1}{\sqrt{2}}\right)\right)^{2}\right]=-l
$$

onde $l=\frac{1}{2} \ln (1+\sqrt{2})[-\sqrt{2}+3 \ln (1+\sqrt{2})]$ é o mesmo que no caso dnoidal estudado acima. Segue de $(6.9)$ e da continuidade das funções $k \longmapsto d n^{-1}\left(\frac{1}{\sqrt{2}} ; k\right)$ e $k \longmapsto$ $\sqrt{2 k^{2}-1}$

$$
\lim _{k \rightarrow 1^{-}} \frac{\omega(k)-\omega^{*}}{Z(k)}=-\frac{1}{\sqrt{2} \operatorname{csech}^{-1}\left(\frac{1}{\sqrt{2}}\right)}(-l)=\frac{l}{c \sqrt{2} \operatorname{sech}^{-1}\left(\frac{1}{\sqrt{2}}\right)}>0 .
$$


Observe que se $\omega_{d n}$ e $\omega_{c n}$ denotam as velocidades para o perfil dnoidal no Teorema 3.6 e cnoidal no Teorema 3.10, respectivamente, então obtemos as relações

$$
\omega_{c n}<\omega^{*}<\omega_{d n}
$$

Portanto, os quocientes $\frac{\omega_{d n}-\omega^{*}}{Z(k)}($ para $Z>0)$ e $\frac{\omega_{c n}-\omega^{*}}{Z(k)}($ para $Z<0)$ são em ambos os casos positivos. Finalmente, no caso $Z<0$ concluímos

$$
\lim _{k \rightarrow 1^{-}} \frac{\omega(k)-\omega^{*}}{Z(k)}\left\|\Psi^{\prime}\right\|^{2}-2\left[\Psi^{\prime}(c)\right]^{2}=\frac{\left(\operatorname{sech}^{-1}\left(\frac{1}{\sqrt{2}}\right)\right)^{2}}{c^{4}}\left[\frac{4 l}{3 \sqrt{2}}-\left(\operatorname{sech}^{-1}\left(\frac{1}{\sqrt{2}}\right)\right)^{2}\right]=L<0 .
$$

Isto prova a proposição.

Corolário 6.2 Para $|Z|$ pequeno e para $\omega=\omega(k)$ a igualdade

$$
\beta\left\|\Psi^{\prime}\right\|^{2}=\frac{\omega-\omega^{*}}{Z}\left\|\Psi^{\prime}\right\|^{2}-2\left[\Psi^{\prime}(c)\right]^{2}+O(Z),
$$

implica que $\beta<0$.

Demonstração. Consideremos o caso $Z>0$. Então, para $h(Z)=O(Z)$, existe $M>0 \mathrm{com}|h(Z)| \leqq M Z$. Da proposição 6.1 temos, para $\epsilon<-L / 2$ que

$$
\beta\left\|\Psi^{\prime}\right\|^{2}<L+\epsilon+h(Z)<L+\epsilon+M Z,
$$

para $k \in(1-\delta, 1)$ e $\delta>0$. A relação $Z=\sqrt{2 \omega} \frac{1-k^{2}}{2-k^{2}}$ implica que $k^{2}=1+\frac{Z}{Z-\sqrt{2 \omega}}$. Então, já que $Z<\sqrt{2 \omega}$; obtemos $k^{2}>1-\delta$ se e somente se $Z<\frac{\delta}{\delta+1} \sqrt{2 \omega}$. Como $\omega(k)>\frac{\pi^{2}}{8 c^{2}}$, obtemos para $Z$ pequeno com

$$
Z<\min \left\{-\frac{L}{2 M}, \frac{\pi}{c} \frac{\sqrt{2}}{4} \frac{\delta}{\delta+1}\right\}
$$

que a relacão (6.13) implica $\beta\left\|\Psi^{\prime}\right\|^{2}<\frac{L}{2}+\epsilon<0$. Logo, $\beta<0$. O caso $Z<0$ é similar. Isto prova o Corolário.

A seguir, daremos uma ideia da afirmação de que para dois pontos de interação, com $Z \neq 0$ fixo; o operador

$$
-\frac{d^{2}}{d x^{2}}+\gamma \delta_{-c}-Z \delta_{c}
$$

pode ser visto como uma família de extensões autoadjuntas associada ao operador simétrico

$$
\left\{\begin{aligned}
-A_{Z} & =-\frac{d^{2}}{d x^{2}} \\
D\left(-A_{Z}\right) & =\left\{\zeta \in H^{1}(\mathbb{R}) \cap H^{2}(\mathbb{R}-\{c\}): \zeta(-c)=0, \zeta^{\prime}(c+)-\zeta^{\prime}(c-)=-Z \zeta(c)\right\} .
\end{aligned}\right.
$$

Este resultado foi usado na Proposição (4.4).

Lema 6.3 Seja $A=-\frac{d^{2}}{d x^{2}}$. A restrição $-\left.A_{Z} \equiv A\right|_{D\left(-A_{Z}\right)}$, com $D\left(-A_{Z}\right)$ dado por (6.15), é um operador simétrico, densamente definido em $L^{2}(\mathbb{R})$ com indices de deficiência $(1,1)$. A saber, os elementos de deficiência estão dados pelas fórmulas

$$
\begin{cases}\text { para } \lambda=i, & g_{i} \equiv(A-i)^{-1} \delta_{-c} \\ \text { para } \lambda=-i, & g_{-i} \equiv(A+i)^{-1} \delta_{-c}\end{cases}
$$

$g_{ \pm i} \in D\left(-A_{Z}^{*}\right) e-A_{Z}{ }^{*} g_{ \pm i}= \pm i g_{ \pm i}$. Mais ainda, $n_{+}\left(-A_{Z}\right)=n_{-}\left(-A_{Z}\right)=1$. 
Demonstração. A prova segue as mesmas linhas do Lemma 1.2.3 em Albeverio\&Kurasov [6].

Presentamos explícitamente agora todas as extensões autoadjuntas do operador simétrico $-A_{Z}$, as quais estarão parametrizadas pelo número $\gamma$. Da teoria de Von Neumann para extensões autoadjuntas de operadores simétricos (ver [37]) obtemos que todas as extensões simétricas de $-A_{Z}$ são autoadjuntas e coincidem com a restrição do operador $-A_{Z}{ }^{*}$. Além do mais, para $\theta \in[0,2 \pi)$ a extensão autoadjunta, $-A_{Z}(\theta)$, de $-A_{Z}$ está definida como segue:

$$
\left\{\begin{array}{l}
D\left(-A_{Z}(\theta)\right)=\left\{\psi+\lambda g_{i}+\lambda e^{i \theta} g_{-i}: \psi \in D\left(-A_{Z}\right), \lambda \in \mathbb{C}\right\}, \\
-A_{Z}(\theta)\left(\psi+\lambda g_{i}+\lambda e^{i \theta} g_{-i}\right)=-A_{Z}^{*}\left(\psi+\lambda g_{i}+\lambda e^{i \theta} g_{-i}\right)=-A_{Z} \psi+i \lambda g_{i}-i \lambda e^{i \theta} g_{-i} .
\end{array}\right.
$$

Agora, da fórmula explícita dos elementos de deficiência $g_{ \pm i}$,

$$
g_{ \pm i}(x)=\frac{i}{2 \sqrt{ \pm i}} e^{i \sqrt{ \pm i}|x+c|}, \quad \operatorname{Im} \sqrt{ \pm i}>0
$$

temos as seguintes expressões básicas para $\zeta \in D\left(-A_{Z}(\theta)\right)$,

$$
\zeta^{\prime}(-c+)-\zeta^{\prime}(-c-)=-\lambda\left(1+e^{i \theta}\right) .
$$

O passo seguinte é encontrarmos $\gamma$ tal que $\gamma \zeta(-c)=-\lambda\left(1+e^{i \theta}\right)$. De fato, depois de alguns cálculos encontramos a fórmula (ver [3])

$$
\gamma(\theta)=\frac{-2 \cos (\theta / 2)}{\cos \left(\frac{\theta}{2}-\frac{\pi}{4}\right)}
$$

Portanto, se $\theta$ varia em $[0,2 \pi), \gamma=\gamma(\theta)$ varia em $\mathbb{R} \cup\{+\infty\}$. Para o único ponto $\theta_{0} \in[0,2 \pi)$ tal que $\cos \left(\frac{\theta}{2}-\frac{\pi}{4}\right)=0$ obtemos $\lim _{\theta \uparrow \theta_{0}} \gamma(\theta)=+\infty$.

Desta forma, parametrizamos todas as extensões autoadjuntas de $-A_{Z}$ com a ajuda de $\gamma$. Temos então,

Proposição 6.4 Seja $Z \in \mathbb{R}$ fixo. Todas as extensões autoadjuntas de $-A_{Z}$ estão dadas, para $-\infty<\gamma \leqq+\infty$ pelas fórmulas: para $\gamma \in(-\infty, \infty)$

$$
\left\{\begin{aligned}
-\Delta_{-Z, \gamma} & =-\frac{d^{2}}{d x^{2}} \\
D\left(-\Delta_{-Z, \gamma}\right) & =\left\{\zeta \in H^{1}(\mathbb{R}) \cap H^{2}(\mathbb{R}-\{ \pm c\}): \zeta^{\prime}(c+)-\zeta^{\prime}(c-)=-Z \zeta(c),\right. \\
\left.\zeta^{\prime}(-c+)-\zeta^{\prime}(-c-)=\gamma \zeta(-c)\right\} . &
\end{aligned}\right.
$$

$O$ caso especial em que $\gamma=0$ dá origem ao operador com um ponto de interação, $-\Delta_{-Z}=-\frac{d^{2}}{d x^{2}}-Z \delta_{c}$ em $L^{2}(\mathbb{R})$,

$$
\left\{\begin{aligned}
-\Delta_{-Z} & =-\frac{d^{2}}{d x^{2}} \\
D\left(-\Delta_{-Z}\right) & =\left\{\zeta \in H^{1}(\mathbb{R}) \cap H^{2}(\mathbb{R}-\{c\}): \zeta^{\prime}(c+)-\zeta^{\prime}(c-)=-Z \zeta(c)\right\} .
\end{aligned}\right.
$$

Demonstração. Pelos argumentos esboçados acima, obtemos fácilmente que $-A_{Z}(\theta) \subset-\Delta_{-Z, \gamma}$, com $\gamma=\gamma(\theta)$ dado em (6.19). Mas $-\Delta_{-Z, \gamma}$ é simétrico no correspondente domínio $D\left(-\Delta_{-Z, \gamma}\right)$, para todo $-\infty<\gamma \leqq+\infty$, o que implica a relação $-A_{Z}(\theta) \subset-\Delta_{-Z, \gamma} \subset\left(-\Delta_{-Z, \gamma}\right)^{*} \subset-A_{Z}(\theta)$. Isto finaliza a Proposição. 


\section{Bibliografia}

[1] Adami, R., Noja, D. and Visciglia, N., Constrained energy minimization and ground states for NLS with point defects. Discrete Contin. Dyn. Syst. Ser. B 18, no. 5, 1155-1188, (2013).

[2] Albiez, M., Gati, R., Fölling, J., Hunsmann, S., Matteo, C. and Oberthaler M. Direct Observation of Tunneling and Nonlinear Self-Trapping in a Single Bosonic Josephson Junction, Phys. Rev. Lett. 95, 010402 (2005).

[3] Alveberio, S, Gesztesy, F., Krohn, R. and holden, H. Solvable Models in quantum mechanics, AMS Chelsea publishing, (2004).

[4] ANGulo, J. Non-Linear stability of periodic travelling-wave solutions for the Schrödinger and modified Korteweg-de Vries equation, Journal Differential Equations, 1-30, 236, (2007).

[5] Angulo, J. and POnCE, G. The nonlinear Schrödinger equation with a periodic $\delta$-interaction, Bull. Braz. Math. Soc., New Series 44(3), 497-551, (2013).

[6] Albeverio, S. and Kurasov, P., Singular Perturbations of Differential Operators, London Mathematical Society, Lecture Note Series, 271, Cambridge University Press, (2000).

[7] BAmbusi, D. and SACCHETt, A. Exponential times in the onedimensional Gross-Pitaevskii equation with multiple well potential, Comm. Math. Phys. 275, 1-36 (2007).

[8] Berezin, F.A., Shubin, M.A. The Schrödinger Equation. Mathematics and its Applications (Soviet Series), Kluwer Academic Publisher Group, Dordrecht, (1991).

[9] Boyd, R. W., Nonlinear Optics, 3rd edition, Academic Press, (2008).

[10] BYRD, P.F. and FRIEDMAN, M.D., Handbook of Elliptic Integrals for Engineers and Scientist, second ed., Springer-Verlag, New York, (1971).

[11] CAmbournac, C., Sylvestre, T., Maillote, H., VAnderlinden B., KOCKAERT, P., EMPLit, PH. and HAELTERMAN M. Symmetry-Breaking 
Instability of Multimode Vector Solitons, Phys. Rev. Lett. 89, 083901, (2002).

[12] Caudrelier, V., Mintchev, M., and Ragoucy, E., Solving the quantum non-linear Schrödinger equation with $\delta$-type impurity, J. Math. Phys. 46 (4), 042703-1-24, (2005).

[13] Cazenave, T., Lions, P. L.Orbital stability of standing waves for some nonlinear Schrödinger equations, Commun. Math. Phys., 85, pp. 549-561, (1982).

[14] Cazenave, T. Semilinear Schrödinger Equations, Courant Lecture Notes in Mathematics, 10, (2003).

[15] Coddingtong, E.A., Levinson, N. Theory of Ordinary Differential Equations, Mc Graw Hill, New-York,Toronto, London, (1955).

[16] Datchev, K. and Holmer, J., Fast soliton scattering by attractive delta impurities, Comm. Partial Differential Equations 34, no. 7-9, 1074-1113, (2009).

[17] Dalfovo, F., Giorgini, S., PITAEVSkit, L.P. and STRINGARI, S. Theory of Bose-Einstein condensation in trapped gases, Rev. Mod. Phys. 71, 463-512, (1999).

[18] Davis, K. B., Mewes, M.O., Andrews, M. R., van Druten, N. J., Durfee, D.S., Kurn, D.M. and Ketterle, W., Bose-Einstein condensation in gas of sodium atoms, Phys. Rev. Lett., 74(22), 39693973, (1995).

[19] Fukuizumi, R., OHTA, M. and OzAWA, T. Nonlinear Schrödinger equation with a point defect, Ann. I. H. Poincaré- AN 25, 837-845, (2008).

[20] FUKUizumi, R., JEANJEAN, L. Stability of standing waves for a nonlinear Schrödinger equation with a repulsive Dirac delta potential. Discrete and Continuous Dynamical System, pp. 121-136, (2008).

[21] Goodman, R. H., Holmes, J. and Weinstein, M., Strong NLS soliton-defect interactions, Phys. D 192 (2004), 215-248.

[22] Goodman, R. H., Marzuola, J. and Weinstein, M. I., Self-trapping and Josephson tunneling solutions to the nonlinear Schrödinger/GrossPitaevskii equation. Discrete Contin. Dyn. Syst. 35, no. 1, 225Đ246, (2015).

[23] Grillakis, M., Shatah, J. and Strauss, W. Stability theory of solitary waves in the presence of symmetry I, J. Funct. Anal., 74, pp. 
160-197, (1987).

[24] Grillakis, M., Shatah, J. and Strauss, W., Stability theory of solitary waves in the presence of symmetry II, J. Funct. Anal., 94, pp. 308-348, (1990).

[25] HAYATA, K. and Koshiba, M., Self-localization and spontaneous symmetry breaking of optical fields propagating in strongly nonlinear channel waveguides: limitations of the scalar field approximation, J. Opt. Soc. Am. B 9, 1362-1368, (1992).

[26] Holmer, J., Marzuola, J. and Zworski, M., Fast soliton scattering by delta impurities, Comm. Math. Phys., 274(91), 187-216, (2007).

[27] Holmer, J., Marzuola, J. and Zworski, M., Soliton alignedting by external delta potentials, J. Nonlinear Sci., 17(4), 349-367, (2007).

[28] Holmer, J. and Zworski, M., Slow soliton interaction with external delta potentials, J. Modern Dynam., 1, 689-718, (2007).

[29] Holmer, J. and Zworski, M., Soliton interaction with slowly varying potentials, IMRN, 2008, Article ID rnn026, 36 pages (2008).

[30] Jackson, R.K. and Weinstein, M.I., Geometric Analysis of Bifurcation and Symmetry Breaking in a Gross-Pitaevskii equation, Journal of Statistical Physics, Vol. 116, Nos. 114, August (2004).

[31] Kato,T.Perturbation theory for linear operators, second ed., in: Grundlehren der Mathematischen Wissenschaften, Band 132, SpringerVerlag, Berlin, Heidelberg, New York, (1980).

[32] KIRR, E.W., KEVREKIDIS, F.G., SHILZERMAN E. and WEINSTEIN M.I., Symmetry breaking bifurcation in Nonlinear Schrödinger/GrossPitaevskii Equations, SIAM J. Math. Anal. 40, 566-604 (2008).

[33] KOVǍ̌́lK, H. and SACCHETTI, A. A nonlinear Schrödinger equation with two symmetric point interactions on one dimension, Journal of Physics A: Mathematical and Theoretical. 43, 155205, (2010).

[34] Newell, A.C. and Moloney, J. V., Nonlinear Optics, Advanced Book Program, Westview Press, (2003).

[35] Le Coz, S., Fukuizumi, R., Fibich, G., KSherim, B. and Sivan, Y. Instability of bound states of a nonlinear Schrödinger equation with a Dirac potential, Physica D 237, 1103-1128, (2008).

[36] Raghavan, S., SMerzi, A., FAntoni, S. and Shenoy, s.R., Coherent oscillations between two weakly coupled Bose-Einstein condensates: 
Josephson effects, oscillations, and macroscopic quantum self-trapping, Phys. Rev. A 59, 620-633 (1999).

[37] Reed, M. and Simon, B. Methods of Modern Mathematical Physics IV: Analysis of operators. New York- San Francisco-London: Academic Press. XV, (1978).

[38] SACCHETTI, A., Nonlinear double-well Schrödinger equations in the semiclassical limit, J. Stat. Phys. 119, 1347-1382 (2005).

[39] SACCHETTI, A., Nonlinear time-dependent Schrödinger equations: the Gross-Pitaevskii equation with double-well potential, J. Evol. Eq. 4, 345-369 (2004).

[40] Sulem, C. and Sulem, P-L., Nonlinear Schrödinger Equations: Self-Focusing and Wave Collapse, Applied Mathematical Sciences, vol. 139, Springer, New York, (1999).

[41] Weinstein, M.I. Nonlinear Schrödinger equation and sharp interpolation estimates. Comm. Math. Phys., 87, pp. 567-576, (1983)

[42] Weinstein, M.I.Modulational stability of ground states of nonlinear Schrödinger equations, SIAM J. Math. Anal. 16, pp. 472-491, (1985). 